B O N N E V I L L E Habitat Evaluation Procedures (HEP) Report;

\author{
Yakama Nation Wildlife Management Areas
}

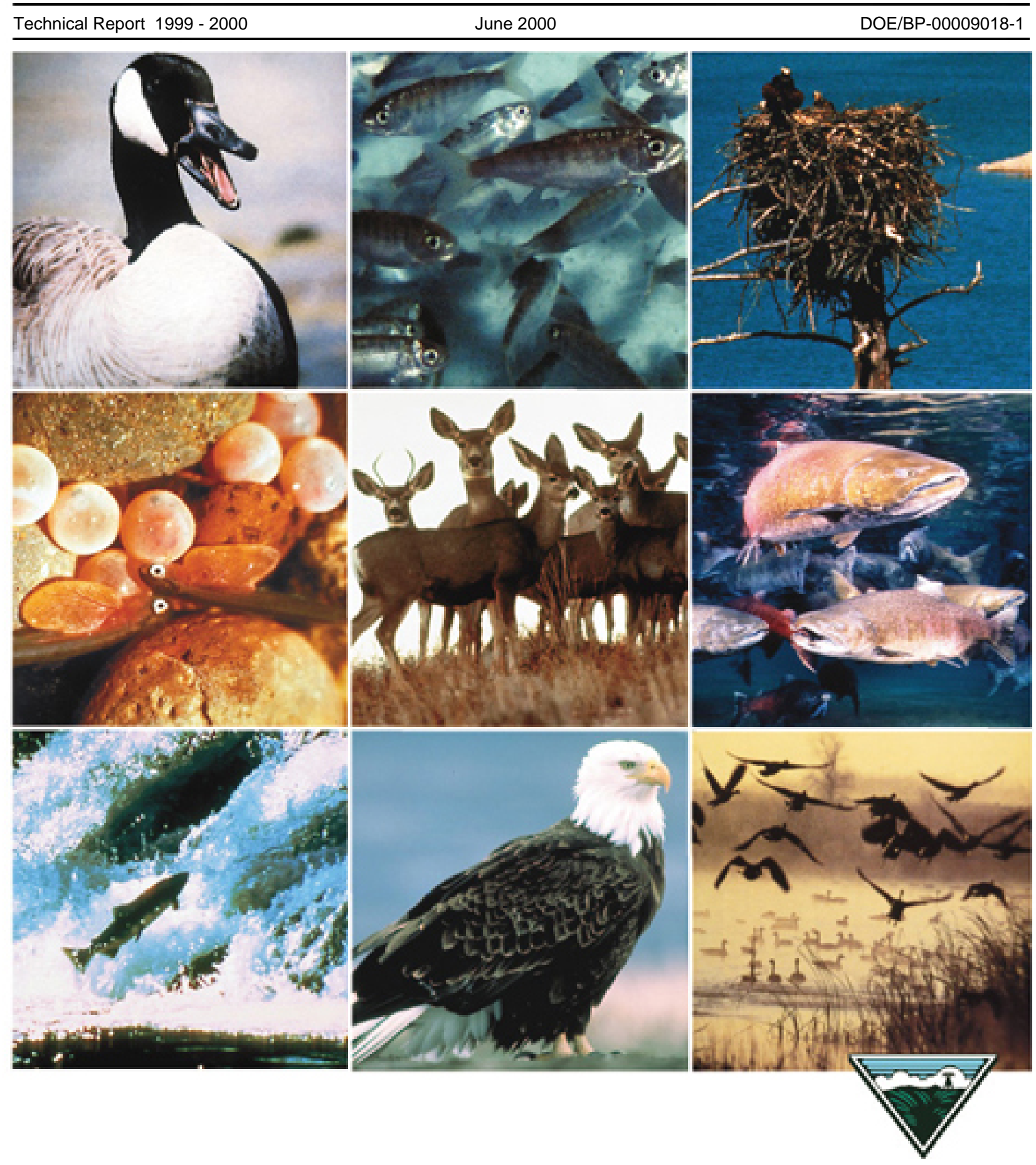


This Document should be cited as follows:

Raedeke, Kenneth, Dorothy Raedeke, "Habitat Evaluation Procedures (HEP) Report;; Yakama

Nation Wildlife Management Areas", 1999-2000 Technical Report, Project No. 199206200, 107

electronic pages, (BPA Report DOE/BP-00009018-1)

Bonneville Power Administration

P.O. Box 3621

Portland, OR 97208

This report was funded by the Bonneville Power Administration (BPA), U.S. Department of Energy, as part of BPA's program to protect, mitigate, and enhance fish and wildlife affected by the development and operation of hydroelectric facilities on the Columbia River and its tributaries. The views in this report are the author's and do not necessarily represent the views of BPA. 


\section{HABITAT EVALUATION PROCEDURES}

Wildlife Management Areas Yakama Nation, Washington

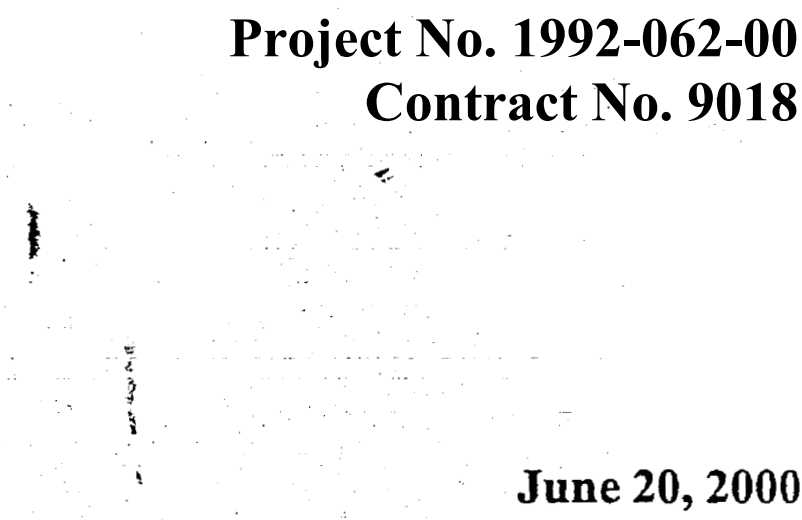


Report To:

Title:

Project Number:

Prepared By:

Date:
Yakama Nation

Fish and Wildlife Program

P.O. Box 151

Toppenish, Washington 98948

Habitat Evaluation Procedures

Wildlife Management Areas

Yakama Nation, Washington

99023-001

Raedeke Associates, Inc. 5711 Northeast 63rd Street Seattle, Washington 98115

(206) 525-8122

June 20, 2000

\section{RAEDEKE ASSOCIATES, INC}


Principals:

Project Personnel:
Kenneth J. Raedeke, Ph.D.

Certified Senior Ecologist, ESA

Dorothy A. Milligan Raedeke, M.S.

Wildlife Biologist

Richard W. Lundquist, M.S.

Associate Wildlife Biologist

Gail Livingstone, BSLA

Resource Planner

Kim Clousing, B.A.

Editing/ Document Production 


\section{TABLE OF CONTENTS}

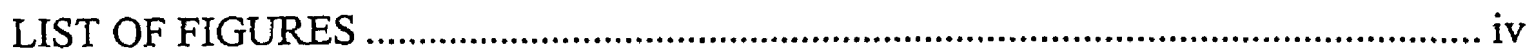

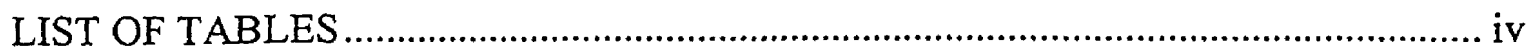

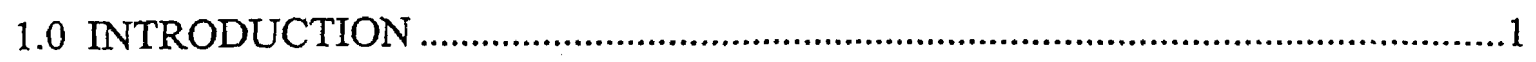

1.1 Background on Wildlife Mitigation Areas ...........................................................

1.2 Scope and Objectives.....................................................................................

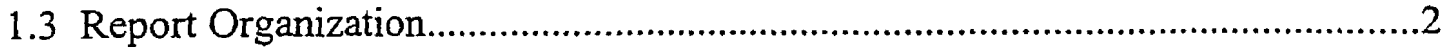

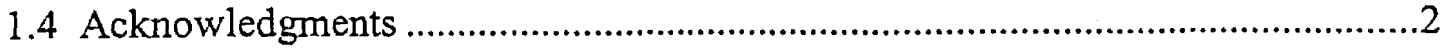

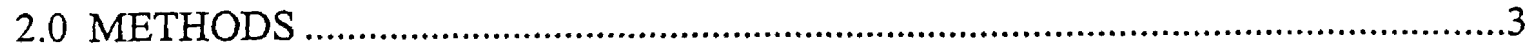

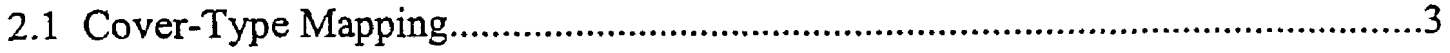

2.2 Wildlife Species and Habitat Model Selection ......................................................

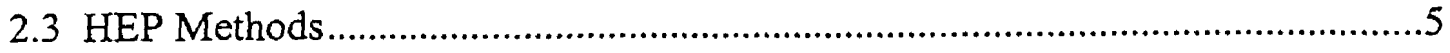

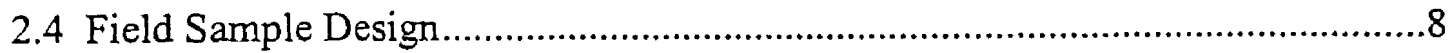

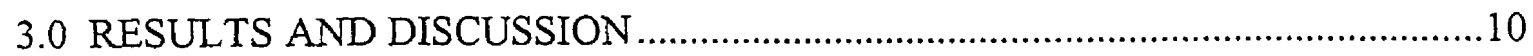

3.1 Evaluation of Current Habitat Conditions ..........................................................10

3.2 Evaluation of HEP Methodologies ............................................................... 10

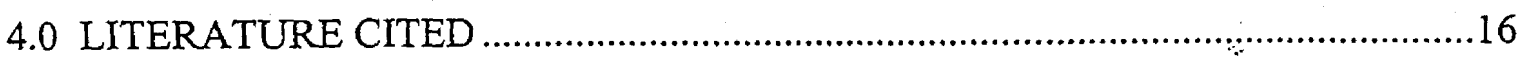

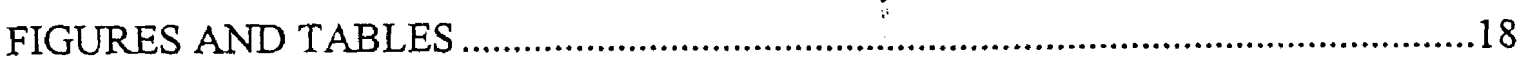

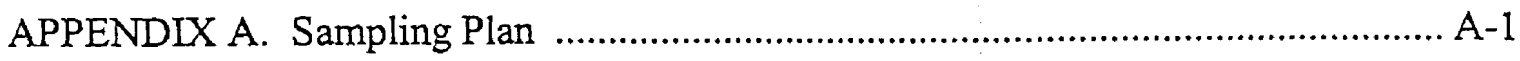

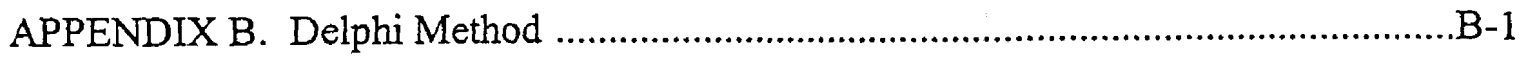

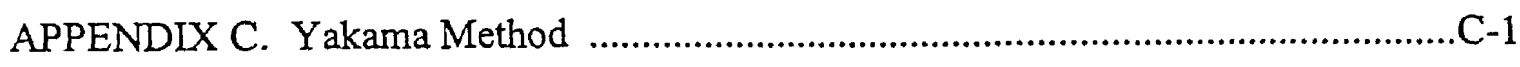




\section{LIST OF FIGURES}

Figure

Page

1. Regional map, Yakama Nation Wildlife Management Areas

19

\section{LIST OF TABLES}

Table

Page

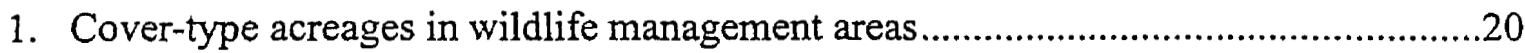

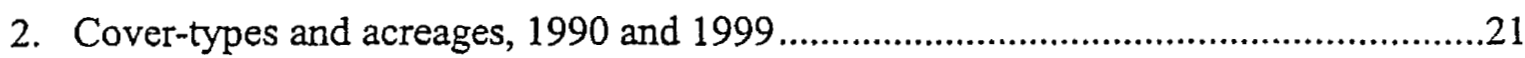

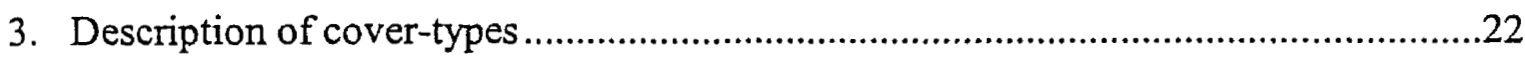

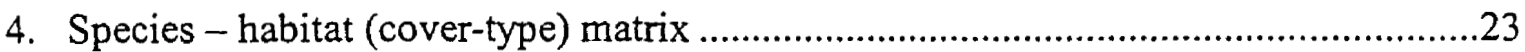

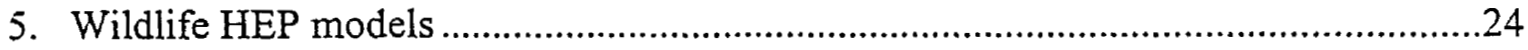

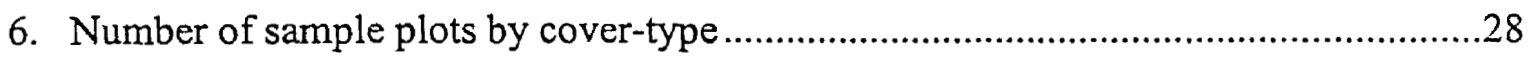

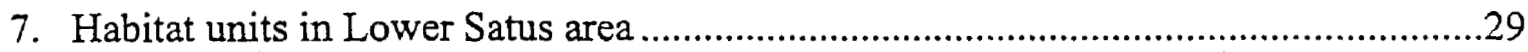

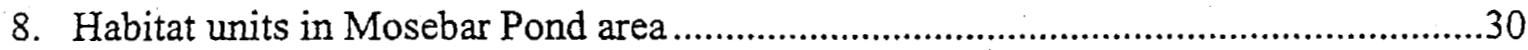

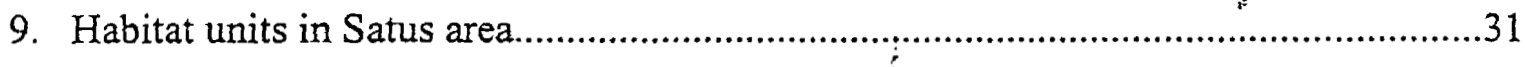

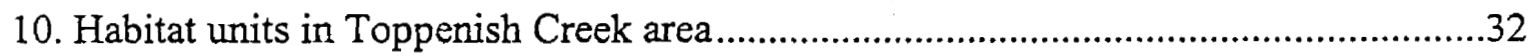

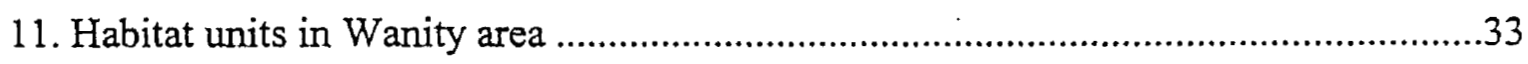

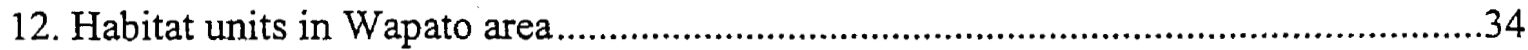

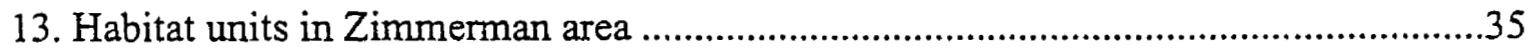

14. Habitat units in all wildlife management areas....................................................36

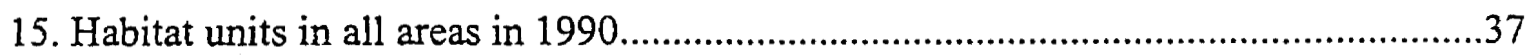

16. Cover-types acreages by wildlife management areas in 1999 ..................................38

17. Average HSI scores with Delphi method..............................................................39

18. Average HSI scores with Yakama method ..........................................................40

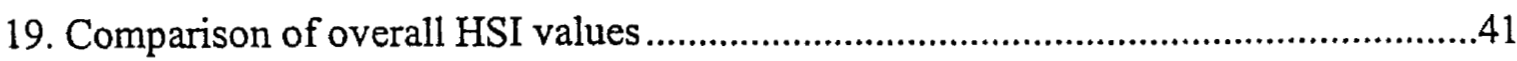

20. Comparison of effort required for HEP analysis ..............................................42 


\subsection{INTRODUCTION}

\subsection{BaCkground ON Wildlife MaNagement AREAS}

Construction of the Dalles, Bonneville, McNary, and John Day Dams on the Columbia River by the federal government resulted in a substantial loss of riparian bottomland along the Columbia River. Impacts associated with the Mid-Columbia Projects were assessed for several wildlife species using the Habitat Evaluation Procedures (HEP) developed by the U.S. Fish and Wildlife Service (USDI-FWS 1980). The studies documented the loss of riparian habitat and established a baseline against which mitigation measures could be developed (USDI-FWS 1990 and USDE-BPA 1990). The impact assessments established a mitigation goal, a portion of which would be satisfied by the creation, restoration, and enhancement of riparian lands on tributaries to the Columbia River, including the Yakima Valley.

The Yakama Nation (YN), the Northwest Power Planning Council, and the Bonneville Power Administration have agreed that the Yakama Nation would be funded to implement habitat restoration on lands within and adjacent to their reservation. Some of the targeted lands are owned by the Yakama Nation, some are trust lands, and some lands have been in private ownership.

Since the early 1990s, the Yakama Nation has been in the process of assembling riparian lands into Wildlife Management Areas, and restoring natural hydrology and natural covertypes on these lands. The Northwest Power Planning Council, through the Bonneville Power Administration, has supported the program.

The Yakama Nation's wildlife mitigation lands are located in the Yakima Valley, on various parcels on the south side of the Yakima River from the town of Wapato, downstream to the town of Mabton, and adjacent to portions of Toppenish Creek and Satus Creek south of the town of Toppenish (Figure 1). The Yakama Nation has identified specific parcels of riparian land adjacent to the Yakima River, Toppenish, and Satus Creek for management as Wildlife Management Areas (Figure 1). The acquisition and management of these lands is, in part, mitigation for wildlife and habitat impacts that have resulted from the construction and operation of the Mid-Columbia Dams.

\subsection{SCOPE AND ObJECTIVES}

HEP studies were performed by the Yakama Nation in 1990 (Bich et al. 1991) to establish baseline conditions and inventory wildlife habitat at the initiation of the restoration project. The 1990 HEP used a simplified version of the HEP to quantify baseline conditions. 
The present assessment is designed to evaluate the progress of the mitigation plan in meeting its stated goals. The 1999 HEP assessment has two distinct tasks:

1. Evaluation of the mitigation plan as currently implemented using the simplified YN HEP methodologies for the Wildlife Management Areas.

2. Evaluation of the simplified YN HEP methodologies as a means of measuring mitigation progress.

\subsection{REPORT ORganization}

This report has been organized to present a minimum of descriptive and analytic text, figures, and tables in the main body of the report. There is, however, a great deal of documentation, data, and analysis that supports the text. Figures and tables associated with the text and part of the main body of the report are identified by a simple table or figure number. Background and supporting material is found in the appendices. Appendix A is general material related to the sampling, Appendix B describes the Delphi Method sampling and analysis, and Appendix $\mathrm{C}$ describes the Yakama Method.

Appendix figures and tables are identified by the appendix prefix; for example, Table A-4 or C-7. Since the text may include references to main-body figures and tables, as well as appendix figures and tables, care must be used when reading the text. -

\subsection{ACKNOWLEDGMENTS}

We appreciate the assistance provided by Mr. Tracy Hames, Project Manager for the Yakama Nation and many other members of the Yakama Nation, and the field assistance provided by persons from various tribes, and federal, state, and local agencies. See Appendix Table A-1 for a list of participants and Table A-2 for the dates on which they participated. Paul Ashley, Washington Department of Fish and Wildlife (WDFW), supervised participants in the sampling and analysis of the data we gathered using the Department's procedures. 


\subsection{METHODS}

To evaluate the success of the YN mitigation program to date, the simplified HEP used in the original analysis was repeated on the lands that have been incorporated into the plan. This allows comparisons with the baseline conditions and the current conditions using the same "currency" for the evaluation.

The simplified YN HEP approach was based on direct observations of habitat conditions and variables in the Habitat Suitability Index (HSI) models for each species, but not detailed field measurements of the variables in the HSI models.

The 1999 HEP evaluation used the inter-agency field assessment approach, with most HSI evaluations completed in the field as described in Bich et al. (1991). The same evaluation species and species models were used. However, the 1999 evaluations were conducted only on the lands that have been incorporated into the Yakama's Wildlife Management Areas. HEP scores were calculated for each mitigation unit, and the total for all units summed for comparison to the projected mitigation scores in the 1991 plan and the habitat mitigation objectives.

In addition we used the Delphi approach to estimate HSI values for each species by direct observation of overall habitat conditions, without estimation or measurement of individual parameters in the HSI models. A single score for each species for each sample area was estimated by a team member, and the scores for all team members were recorded and averaged to get a final HSI score for that plot. The results of this approach were then compared to the simplified YN method and the field measurements approach.

\subsection{COVER-TYPE MAPPING}

In 1990 (Bich et al. 1991) preliminary cover-type maps were used. Since that time, the Yakama Nation has acquired and now uses an ArcInfo (ENSR) geographic information system (GIS) for mapping and other spatial analysis. This report uses cover-type maps prepared by the Yakama Nation based upon cover-type analysis of recent aerial photographs. During the course of field studies in the summer of 1999 , the preliminary GIS cover-type maps were ground-truthed and refined.

Table 1 provides the area of each cover-type in each of the Wildlife Management Areas based upon the revised GIS cover-type maps. The cover-types roughly follow those used by the Fish and Wildlife Service (USDI-FWS 1990 and USDE-BPA 1990) in their evaluations of impacts caused by development of the Mid-Columbia River Projects. However, the cover-types have been further expanded by the $\mathrm{YN}$ staff to distinguish covertypes that contain a significant coverage by exotic plant species. 
Table 2 gives the simplified cover-type acreages used in the 1999 analysis by Wildlife Management Area. Table 3 provides a description of the cover-types, and the cover-type codes used in the YN computer GIS database and the corresponding U.S. Fish and Wildlife Service codes.

Restoration of a more natural hydrology and replanting of native grasslands has resulted in the replacement of pasture and croplands with natural grasslands and palustrine emergent wetlands since the $1990 \mathrm{HEP}$ was conducted. Some of the shrub-lands that were heavily infested with Russian olive have been cleared of the shrub, which does not change its basic shrub cover-type, but does result in its changing from a shrub-land contaminated with exotics to a native shrub-land.

Substantial changes in other cover-type areas have not occurred because existing covertypes are slowly changing as active agricultural uses, including grazing, have been terminated or reduced, and as more natural conditions evolve. In these areas, changes are in the composition and quality of cover-types and not in their areas. As restoration of natural communities is a long-term process, the benefits of restoration are only beginning to appear.

\subsection{Wildlife Species and Habitat Model Selection}

In a HEP analysis (USDI-FWS 1980), the habitat (cover-types) is assessed for representative wildlife species. Species are selected to represent various guilds, foraging types, and use of various cover-types. For each species, for each cover-type that it uses, an assessment of habitat quality is determined. The descriptor of habitat quality is the Habitat Suitability Index (HSI). For each species for each used habitat, a HSI value is determined. The HSI value is multiplied by the habitat (cover-type) area to calculate the dimensionless Habitat Unit (HU).

In both the $1990 \mathrm{HEP}$ study (Bich et al. 1991) and this HEP study, the species that are used follow those used in the baseline studies on the mainstem Columbia River (USDI-FWS 1990 and USDE-BPA 1990). In those studies, the investigators used a set of birds and mammals that reflected the use of various cover-types and represented a number of foraging guilds, and for which there were HEP models. Table 4 provides the species used in the analysis, the cover-types for which they were analyzed, and the manner in which they use the cover-type; for example, nesting, foraging, and/or hiding.

It should be noted that many of the species listed in Table 4 occur in many of the other habitats not analyzed in this HEP. As noted above, this analysis follows the protocols and species-habitat matrix of the 1990 HEP. 
In the HEP procedures developed by the FWS, the relationship between a species and its habitat is described and semi-quantified by a word or mathematical model. These models seek to describe habitat conditions for a species using several habitat parameters or descriptors. Ideal habitat for a species has an HSI score of 1.0; habitat of no utility for a species would have an HSI score of 0.0 , and intermediate habitats (or cover-types) would have intermediate HSI scores.

In their 1990 HEP analysis (Bich et al. 1991), the Yakama Nation used the same or similar HEP HSI models as had been used in the earlier Columbia River Studies (USDI-FWS 1990 and USDE-BPA 1990). In some cases the models were modified to better reflect local conditions in the Yakima Valley or were updated with refined models.

Table 5 lists the HEP models that were used in this study, the source of the model, its author, habitat parameters, appropriate habitats for measuring, and the computation procedures used to determine the Habitat Suitability Index (HSI). Table 5 also notes where models have been altered for use in this study based upon our field experience.

\subsection{HEP METHODS}

The 1990 Yakama HEP study method did not directly measure parameters or variables in the field to drive the HSI models. Instead, the Yakama 1990 method estimated the field parameters during visits to representative sample sites in each management area and each cover-type. The parameter values for each species model were estimated, species by species, at each sample plot. The estimated parameters were used to drive the standard models to calculate HSIs for each cover-type for each species (see Bich et al. 1991). Subsequently in this report, Yakama Method will refer to the field estimation of species/cover-type HSIs as described for the $1990 \mathrm{HEP}$ project.

Some reviewers of the 1990 HEP study (Bich et al. 1991) criticized the Yakama Method for estimating the parameters rather than making detailed field measurements to calculate HSI values. For the 1999 HEP analysis, the Yakama Nation proposed to repeat sampling and calculation of HSIs and HUs in the same manner as the 1990 study, but also to sample some sites using field measuring methods. When setting up the 1999 field study, Raedeke Associates, Inc. suggested that it would be useful to also sample using the Delphi method. The Delphi method is an accepted HEP process in which the HSI values for each species are estimated in the field at each sample site. The HSIs are not calculated using a mathematical model, but rather a word model.

In addition, Raedeke Associates, Inc. suggested gathering additional environmental and land use information at each site so that a rich database could be developed, which could subsequently be used to analyze the effectiveness of the mitigation program and to evaluate the HEP methodologies. 


\subsubsection{Yakama Nation Method}

\section{Yakama Method Models and Data Forms}

In 1990, the Yakama Nation staff estimated in the field each of the parameters used in each of the Bluebook HEP models, model by model (Bich et al. 1991). The parameters used in each species model were tabulated and sorted. A list of parameters was arranged that would sample each stratum - forest, shrub, herb - and the parameters that were unique to specific species models. Where possible, species models were modified to use the common set of parameters and to eliminate redundant measures. Table $\mathrm{C}-1$ shows the parameters used to drive the models and Table $\mathrm{C}-2$ shows the ordered list of sample parameters.

Data sheets were prepared for each of several major cover-type groups:

- Forest and Shrub

- Shrub-Steppe-Grassland, Grassland, Agriculture Crop, Agriculture Pasture, Herb

- Lacustrine, Open Water, Riverine, Emergent, Sand-Gravel-Cobble-Mud

Appendix C provides the data sheets as they finally evolved (Tables C-3, C-4, and C-5). The data sheets included the parameters that would be used for each species that would be present in the cover-types, as well as general stand information, start and end times, and the observers. The data sheet provided the step values and required that the observer simply check the step value.

\section{Yakama Method Field Sampling}

Field sampling was conducted during the middle two weeks of August 1999. Yakama sampling was performed following the Delphi sampling at each plot. Each morning and afternoon, the participants would travel to one of the Wildlife Management Areas. The pool of available people was divided into teams of three to five people and each team was assigned some cover-type locations to sample. At the sample plot, the team would inspect the cover-type, discuss the site and note the appropriate parameter step value. Each sample plot was given a unique day/location identifier and subsequently assigned a serial number. The plot identifiers and serial number were the same for both the Delphi plots and the Yakama Plots.

\section{Yakama Method Data Entry and Analysis}

Following ordering, review of entries, and annotation if necessary, the data were entered into an Excel spreadsheet. Entered data included location and other site information, the start time and the ending time, and the parameter step value as represented by the appropriate column number. The spreadsheet was reviewed for consistency and accuracy. Table C-6 is a copy of the master Yakama spreadsheet. 
Table C-7 describes the step value for each parameter for each species. Table C-8 translates the step values into the appropriate variable value (V1, V2, V3, etc.) for each parameter for each species. Table C-6 was modified to include the variables for each species HSI model. Using the Microsoft Excel Choose Function, each column step value was replaced with the appropriate variable value. The portion of the table with the replaced variable values is Table $\mathrm{C}-9$. Table $\mathrm{C}-10$ provides the species HSI values derived for the variable scores for each species for each sample plot.

Habitat Units (HUs) for each species were calculated for each individual wildlife management area by multiplying the HSI scores for the cover-types sampled in that specific area by the habitat acreages in that wildlife management area as given in Table 1. In the case where a particular cover-type was not sampled in a given wildlife management area, the overall average for the entire study site was used.

\subsubsection{Delphi Method}

\section{Delphi Word Models and Data Forms}

For this method, verbal descriptions were prepared for each of the HEP species based upon HEP models, other references, and the substantial experience Yakama Nation biologists have acquired during many years of field work and management activities (Appendix $B$, Table B-1). The descriptions included not only descriptions of ideal habitat, but also descriptions of intermediate habitats. A field data form was prepared that asked for all of the information that was to be recorded in each plot (Table B-2). Participants in the field reviewed the word models for each species and entered the consensus habitat value on the data sheet.

\section{Delphi Field Sampling}

Field sampling was conducted during the middle two weeks of August 1999. Each morning and afternoon, the participants would travel to one of the Wildlife Management Areas. The pool of available people was divided into teams of three to five people and each team was assigned some cover-type locations to sample. At the sample plot, the team would review the species models and discuss conditions at the location and then assign an HSI score. The scores for each species were recorded on the data sheets of that sample plot. Each sample plot was given a unique day/location identifier and subsequently assigned a serial number.

\section{Delphi Data Entry and Analysis}

Following ordering, review of entries, and annotation if necessary, the data was entered into an Excel spreadsheet. Entered data included location and other site information, the start time and the ending time, and the species HSI scores. The spreadsheet was reviewed for consistency and accuracy. Table B-3 is a copy of the master Delphi spreadsheet. Table 
B-3 was sorted by the cover-type row, and then the values for each cover-type for each species were summed and averaged.

For comparison with other HEP methods, the overall HSI score for all habitats for the individual species was calculated. Habitat Units were not calculated for comparison of the Delphi method as this would simply be multiplying the HSI score by a constant, and no new information is provided that would be useful in comparison of methodologies.

\subsubsection{Transect Method}

This method is based on field measurements of all parameters in the HSI models. For a description of the Transect Method see WDFW (1997), Sunnyside Wildlife Area, Habitat Evaluation Procedures, Survey Workbook, prepared by Ashley and Wahlen.

Paul Ashley, WDFW, supervised the participants in the using the transect sampling methods on a limited number of plots. Using the Transect Method, three riparian forest, two riparian shrub, and two shrub-steppe samples were taken. Mr. Ashley had to coach the participants through the more intensive sampling methodologies. The forest stands sampled were also infested with poison ivy so more care had to be taken while sampling.

\subsection{Field SAMPLE DESIGN}

The sample design for this study recorded information for the following factors or strata:

1. HEP Procedure type (Delphi, Yakama, or transiect)

2. Wildlife Management Areas

3. Cover-Types

4. Wildlife Species

Prior to field sampling, proposed sampling methodologies, cover-type definitions, sample data sheets, and sampling protocols were developed. On July 24, 1999, Tracy Hames, Yakama Nation, and Richard Fleming, Raedeke Associates, Inc. visited several of the management areas and reviewed the cover-type mapping, evaluated the proposed models and sampling procedures, and estimated how much time it might take to collect samples.

On August 8, Paul Ashley, Washington Department of Fish and Wildlife, HEP Biologist, joined Fleming and Hames in eastern Washington. Again, cover-type maps were evaluated, sampling procedures were reviewed, and several Wildlife Management Areas were visited to gauge the range of conditions that would be sampled.

Field sampling was conducted over two weeks in August 1999. Yakama Nation biologists and other staff, Raedeke Associates, Inc. staff, and participants from various tribes, federal, 
state, and county agencies assisted. Appendix Table A-1 identifies the participants and Table A-2 indicates the days on which sampling took place and who participated. In all, 19 persons participated in sampling on nine different days. There were approximately 51 person-days of field sampling.

Prior to sampling, estimates were made of the number of samples that would be required to adequately sample both Wildlife Management Areas and cover-types, given usual HEP practices. Estimates were also made of the amount of time that would be required to sample, and travel between the various sample areas scattered over more than 200 square miles, including rest and lunch in what was anticipated to be very warm conditions. Tables A-3, A-4, and A-5 in Appendix A show how these factors were calculated to determine how many person-days would be required. Tracy Hames used these estimates to determine the number of people he would request to assist with the field sampling.

Prior to field sampling, staff reviewed aerial photographs and cover-type maps for each Wildlife Management Area and target numbers of samples for each cover-type. They then marked candidate locations on the aerial photographs and cover-type maps for cover-type sample plots. Plots had to be reasonably accessible by foot from the access roads within the areas. Each day the number of samples in each cover-type and in each management area were entered on a tally sheet (summarized in Table 6) and candidates for sampling on the following day were determined given the anticipated size of the field crew.

To speed the process of field sampling, data sheets were developed that would permit participants with minimal experience to effectively gather data and to record all of the needed information. 


\subsection{RESULTS AND DISCUSSION}

\subsection{Evaluation of Current Habitat Condrtions}

Current habitat conditions were evaluated for each Wildlife Management Area and for the total of all the areas. To calculate the HUs for individual Wildlife Management Areas, the HSI scores for each species for each cover type sampled were multiplied by the acreages in Table 2. When a particular cover-type present in a given area was not sampled in that area, the overall HSI score for all areas was used.

The current habitat units by Wildlife Management Area are given in Tables 7 to 13 . The tables include the HSI scores used in the analysis. The bold HSI scores were from plots sampled in the specific area and the non-bold HSI scores are the overall average HSI scores. The overall total for all Wildlife Management Areas is given in Table 14.

The baseline 1990 habitat conditions in these same Wildlife Management Areas are summarized in Table 15. The table includes totals by species and by cover-type. The HUs were calculated by multiplying the HSI scores from the 1990 study (see Table 6 from Bich et al. 1991) by the reconstructed cover-type acreages in Table 16.

The overall habitat units in 1990 were 22,484 versus 24,568 in 1999 . This represents an increase in habitat units of about 9 percent. This increase is due in part to habitat improvements in the intervening years. However, the total scores are also affected by changes in overall acreages within the areas, improved and revised cover-type mapping in 1999, and revisions in HSI models.

\subsection{Evaluation of HEP Methodologies}

The HEP process is based on the assumption that one can calculate habitat units (HUs) by multiplying an index of habitat quality (HSI values) for a given species by the amount of available habitat. As noted in the HEP documentation (See USFWS [1980] 102 ESM 4.2), "the fundamental step in determining HUs is to estimate or calculate HSIs for each evaluation species" and, "an HSI model can be in graphical, word, or mathematical format" (see USFWS [1980] 103 ESM 1).

The HEP guidelines go on to identify a number of ways to develop HSI scores, including word models (102 ESM 4.2B(2)), word rankings (103 ESM 2.1), indices based on species population measures (103 ESM 2.2), or models with undefined outputs (e.g., quantitative habitat models) (103 ESM 2.3). The HEP Workbook (see Chapter 5) notes that "there is no one correct way to build an HSI model." 
In the present study, we calculated HUs based the following methods:

- Yakama method: a quantitative method where HSI model parameters are estimated in the field and used in the mathematical models to calculate the HSI score

- Transect method: also a quantitative method, but where the model parameters are based on field measurements to calculate HSI scores using the same mathematical models

- Delphi method: a method in which HSI values are derived from word models by a team of experienced biologists

Each of these methods has strengths and weaknesses. In this section, we compare the results of the different methods, using the average HSI score as the basis for the comparisons, and compare the amount of effort (measured in time) to conduct the different methods.

\subsubsection{Comparisons of HSI Scores}

The HSI scores based on the Delphi and Yakama methods are summarized in Tables 17 and 18. The tabled values are average HSI scores by species by cover-type for all plots. Table 19 provides a summary of overall HSI scores by species for the Delphi and Yakama methods.

At this time, we do not have HSI score results for the Transect method for comparison.

Even though we tried to make the data forms as explicit as possible, Delphi method sampling is more subjective than Yakama method sampling. Sampling teams were selected to include staff from the Yakama Nation. These staff persons have many years of experience in wildlife management on the lands we were assessing. Thus, we anticipated that Delphi method sampling might provide a more accurate estimate of habitat value to a species.

Average HSI scores for the Delphi method and the Yakama method show a variable pattern by species (Table 19). Overall, the Delphi scores are $28 \%$ lower than the Yakama method scores. The Delphi scores are substantially lower for the Canada goose, California quail, and mink, while the Delphi scores are higher for the great blue heron and western meadowlark. For the other species, the scores are similar, but sample size is quite small, with the exception of the mallard. 


\subsubsection{Comparisons of Effort Between Methods}

To compare the amount of effort (measured in staff time needed to complete the analysis) we divided the methods into the three major analysis phases, including mobilization and field preparation, field data collection, and computation of scores.

\section{Field Preparation Effort}

The initial task for any HEP analysis would include the following steps:

- project scoping and study plan development

- species selection

- habitat cover-type mapping

- HSI model selection and/or development

- data collection preparations

The amount of effort to complete this phase of a HEP analysis would be identical for all three methods for the first three steps.

HSI model selection and/or development would differ between the Delphi method and the other two. The Yakama method and the Transect method both require mathematical models to calculate the HSI values for each species and cover type. Where models exist, minimal effort is required to obtain the models. However, time would be required to review the models, as the majority of the existing HSI models date to the early $1980 \mathrm{~s}$. If new models need to be developed, we estimate that a minimum of 2 person-days would be required to compile and review the literature, and prepare the model.

For the Delphi method, we estimate that it would require at maximum 2 hours to prepare a word model if an HSI model is in existence, and 4 hours if there is no HSI model.

Preparing field data sheets would again be similar for the Yakama and Transect methods, and would require several person-days. The Delphi method would require several hours to prepare simple data sheets to record the appropriate data.

In summary, the Yakama and Transect methods would require five to ten times more effort to prepare the HSI models and data sheets than would the Delphi method. However, for all methodologies, the habitat cover-type mapping and associated GIS analysis is the most labor intensive task and the amount of required effort is constant across methodologies.

\section{Field Sampling Effort}

Both the Delphi and Yakama sampling methods proceeded quickly in the field once the procedures became familiar. The Yakama Method was almost always faster than the Delphi Method as there was always a discussion within the sampling team to arrive at a 
Delphi HSI value. The Delphi value is a balance of many factors and it was often necessary to review the word models and consider the various factors before a consensus score was decided upon.

In contrast, the Yakama Method required only the field estimation of the various parameter values. The field sheets provided step ranges for parameter values and the appropriate step was simply marked in the field. This usually did not require much discussion within a sampling team, and teams were more comfortable with estimating parameter step values than they were with estimating species HSI values.

The Transect Method sampling required much more time for team members to become familiar with the methodology and much more effort was required to calculate the parameter estimates. The Transect Method of sampling was usually conducted by teams of 6 to 10 persons. The size of the sampling team reflected the greater effort required by the sampling, but also the desire to introduce as many people as possible to the procedure. Teams of trained individuals could probably be reduced to 3 or 4 persons.

The average time to perform a Delphi method sample ( $\mathrm{n}=86 \mathrm{plots})$ was 8.1 minutes while the average time to perform a Yakama method sample $(n=88$ plots) was 4.3 minutes. The average times for Transect method were: (a) riparian forest ( $n=3$ plots) 120 minutes, (b) shrub-steppe-grassland ( $n=2$ plots) 60 minutes, and (c) grassland $(n=1$ plot) 45 minutes.

\section{Computational Effort}

There is no required effort to complete the computations on the Delphi methods, as the field results provide the final HSI values. The Yakama and Transect methods require virtually the same amount of effort to summarize and analyze the field date and compute the final HSI scores.

The calculation of the HSI scores for the Yakama and Transect methods is greatly simplified through the use of spreadsheets that incorporate the HSI quantitative models. As part of this analysis we constructed such spreadsheets, and future analysis for field data would require minimal effort (e.g., several person-days) for even a very large data set. We estimate the construction of the spreadsheets and model development for this project could have been completed in approximately 5 person-days, if the required quantitative models were provided as part of the first step in this process.

Table 20 provides a summary of the required effort to complete a HEP analysis using the three different methodologies. Included is a hypothetical example that has 100 sample points in the analysis, sampling for 10 species by a two-person field team. Where the amount of effort for the three methodologies is the same (e.g., cover-type mapping, GIS analysis), no value is included for simplification. It should be noted that the habitat mapping and GIS would be major components of the effort required for all methods. 
Based on this analysis, the Delphi and Yakama methods are relatively similar in amount of effort required. The Transect method would require more than five times more effort to complete the same number of sample plots as compared to the other two methodologies.

\subsubsection{Discussion of Methodologies}

As noted above, USFWS has clearly stated in the HEP documentation that there is no absolute correct way to derive HSI scores, and as such, the results of one method are no more valid than those based on another method. In our evaluation we are most concerned with the amount of subjectivity, the amount of effort required, and the ability of the method to meaningfully capture the changes in habitat quality over time and/or in response to active habitat management.

Critics of the original 1990 Yakima HEP study suggest that detailed field measurements of HSI model parameters would provide more precise estimates of the habitat suitability than would estimates of the parameter values. Further, supporters of the Transect method for HSI calculation feel that the quantitative models used in the Transect method provide more precise measures of habitat suitability when compared to the word models used in the Delphi method.

The quantitative HSI models do give the impression of precision that may not be justified for several reasons. First, the HSI models are composed of a number of suitability indices, which are models of species response to different habitat factors. For example, the great blue heron HSI model includes models for the parameters: (V1) distance from feeding areas to nest areas; (V2) feeding area quality; (V3) human disturbance; (V4) nest area conditions; (V5) nest disturbance; (V6) distance between potential and active nest sites. In reality, each of the variable models was derived via the Delphi process, as there is no specific research results that provides these specific models (see Stabins and Raedeke 1992). They are the best professional judgment of the individual or team that constructed the model and are a very rough approximation of how they think the animals respond to multiple habitat features in a simultaneous manner.

Second, the final HSI score is derived by some combination of these variables in a simple algebraic formula. Again, in the case of the great blue heron, the HSI score is the geometric mean of all the variables. The ecological basis for this calculation is arguable, as this formula assumes that one variable compensates for another (i.e., you can get moderate HSI when one variable is low and another is high - they cancel each other out).

An inter-agency team that was charged with evaluation of the HSI approach for evaluation of the habitat assessment for the Forest Plan for the Tongass National Forest concluded that 


\section{"while superficially giving the appearance of a model based on cause-and-effect relationships, the critical linkages are abstract and do not have biological or ecological underpinnings" (Hanley et al. 1985).}

Indeed, Hanley et al. (1985) go on to conclude that the fundamental assumption of correlation between model results and habitat capacity is untestable. Roloff and Kerhohan (1999) found that none of the 58 existing HSI models that were tested had been validated, and correlations that have been noted may be spurious.

A second major concern is the amount of effort required for the Transect method versus the others, and the ability to adequately sample the range of variability in individual model parameters and variability within habitat conditions. Clark and Lewis (1983) attributed poor HSI model performance with sampling in a limited range of habitat conditions, and Bender et al. (1996) found poor HSI model performance resulting from inadequate consideration of data variability.

The weakest component of most HEP analyses is inadequate consideration of input data variability and how variability affects final HSI output interpretation (Roloff and Kerhohan 1999). Typically, the resources do not exist to thoroughly sample each habitat cover-type, and capture the variability within these cover-types. Thus, statistical means are often based on small sample sizes. Hence, a method that allows the greatest number of sample plots per unit of effort can provide an estimate with greater reliability than a method that allows few sample plots per unit of effort, even if the method is more quantitative.

For example, in the Transect method in this study, measurements of tree canopy in the North Satus unit averaged 34 percent, with a range of 10 to 70 percent. Based on a sample size of 5 plots, it would be hard to argue that these field measurements would be superior to visual estimates on 25 plots. The latter would be the number of plots that could be visually sampled using the Yakama method with the same amount of effort as the 5 plots using the Transect method.

The strongest argument in favor of the Transect method is repeatability, as trained biologists should be able to make the habitat variable measurements in a repeatable fashion. However, trained observers can also make visual estimates of variables in a repeatable fashion, especially since many of the variables are categorical (see Cook and Stubbendieck 1986).

In summary, while the Transect method would appear to be more precise and repeatable, the underlying assumptions of the quantitative models make them no more reliable than the Delphi models on which they are based. Second, the greater efficiency of collecting data using the Yakama method and the Delphi method allows a much larger number of sample plots, and increased reliability of the final HSI values. 


\subsection{LITERATURE CITED}

Allen, A. 1986. Habitat suitability index model: mink. U.S. Fish and Wildlife Service, revised Bluebook Model. FWS/OBS 82/10.127

Ashley, P. 1999. Mallard HEP Model, Columbia Basin Wildlife Area. Columbia River Wildlife Mitigation Team. $7 \mathrm{pp}$.

Bender, L., G. Roloff, and J. Haufler. 1996. Evaluating confidence intervals for habitat suitability models. Wildlife Society Bulletin 24:347-352.

Bich, J., T. Hames, S. McCorquodale, J. Reichel, and W. Bradley. 1991. The Yakima Indian Nation Wildlife Mitigation Plan for Bonneville, The Dalles, John Day and McNary Dams. Wildlife Resources Management. Prepared for the Northwest Power Planning Council. 62 pp.

Clark, J., and J. Lewis. 1983. A validity test of habitat suitability index model for clapper rail. Proceedings of the Annual Conference of the Southeastern Association of Fish and Wildlife Agencies 37:95-102.

Cook, C., and J. Stubbendieck. 1986. Range research: basic problems and techniques. Society for Range Management, Denver, Colorado. $317 \mathrm{pp}$.

DeWard, B. 1990. Draft Canada Goose habitat suitability index model. Unpublished review copy. Wildlife Resource Management, Yakima Indian Nátion, Toppenish, Washington. 2 pp.

Dorsey, G. 1990. Habitat suitability index models, spotted sandpiper. Willamette Ecoregion [with McNary modifications]. Unpublished review copy, U.S. Fish and Wildlife Service.

Hanley, T., M. Kirchoff, and L. Suring. 1985. Report to the Interagency Wildl. Habitat Committee: eval. of deer models for Southeastern Alaska. Unpubl. report. $10 \mathrm{pp}$.

Rasmussen, L. and P. Wright. 1990. Mallard HEP Model, Columbia Basin Wildlife Area. Revised February 10, 1999. Columbia River Wildlife Mitigation Team.

Roloff, G., and B. Kerhohan. 1999. Evaluating reliability of habitat suitability index models. Wildlife Society Bulletin 27(4):973-985.

Schroeder, R. 1982. Habitat suitability index models: yellow warbler. U.S. Fish and Wildlife Service. FWS/OBS-82/10.27

Schroeder, R. 1983a. Habitat suitability index model: black-capped chickadee. U.S. Fish and Wildlife Service, review copy. FWS/OBS-82/10.37 
Schroeder, R. 1983b. Habitat suitability index model: downy woodpecker. U.S. Fish and Wildlife Service. FWS/OBS-82/10.38

Schroeder, R. and P. Sousa. 1982. Habitat Suitability index model: western meadowlark. U.S. Fish and Wildlife Service. FWS/OBS-82/10/29

Schroeder, R. and Richard. 1978. Habitat Suitability index model: California quail. U.S. Fish and Wildlife Service.

Short, H. and R. Cooper. 1985. Habitat suitability index model: great blue heron. U.S. Fish and Wildlife Service. FWS/OBS-82/10.99

Stabins. H., and K. Raedeke. 1992. Status of great blue heron nesting colonies in King County, Washington. Northwest Sciences 66(2):126.

U.S. Department of Interior (U.S. Fish and Wildlife Service). 1980. Habitat evaluation procedures (HEP). Ecological services Manual 102. Division of Ecological Services, Washington, D.C.

U.S. Department of Interior (U.S. Fish and Wildlife Service). 1990. Wildlife Impact Assessment, The Dalles Project, Oregon and Washington, Annual Report 1989. Prepared by Larry Rasmussen and Patrick Wright, Portland Field Station, US Fish and Wildlife Service for U.S. Department of Energy (Bonneville Power Administration).

U.S. Department of Energy (Bonneville Power Administration) and U.S. Department of Interior (US Fish and wildlife Service). 1990. Wildlife Impact Assessment, Bonneville, McNary, The Dales, and John Day Projects. Annual Reports. 46 pp.

Washington Department of Fish and Wildlife. 1997. Sunnyside Wildlife Area, Habitat Evaluation Procedures, Survey Workbook. Columbia River Wildlife Management Team. 
FIGURES AND TABLES 


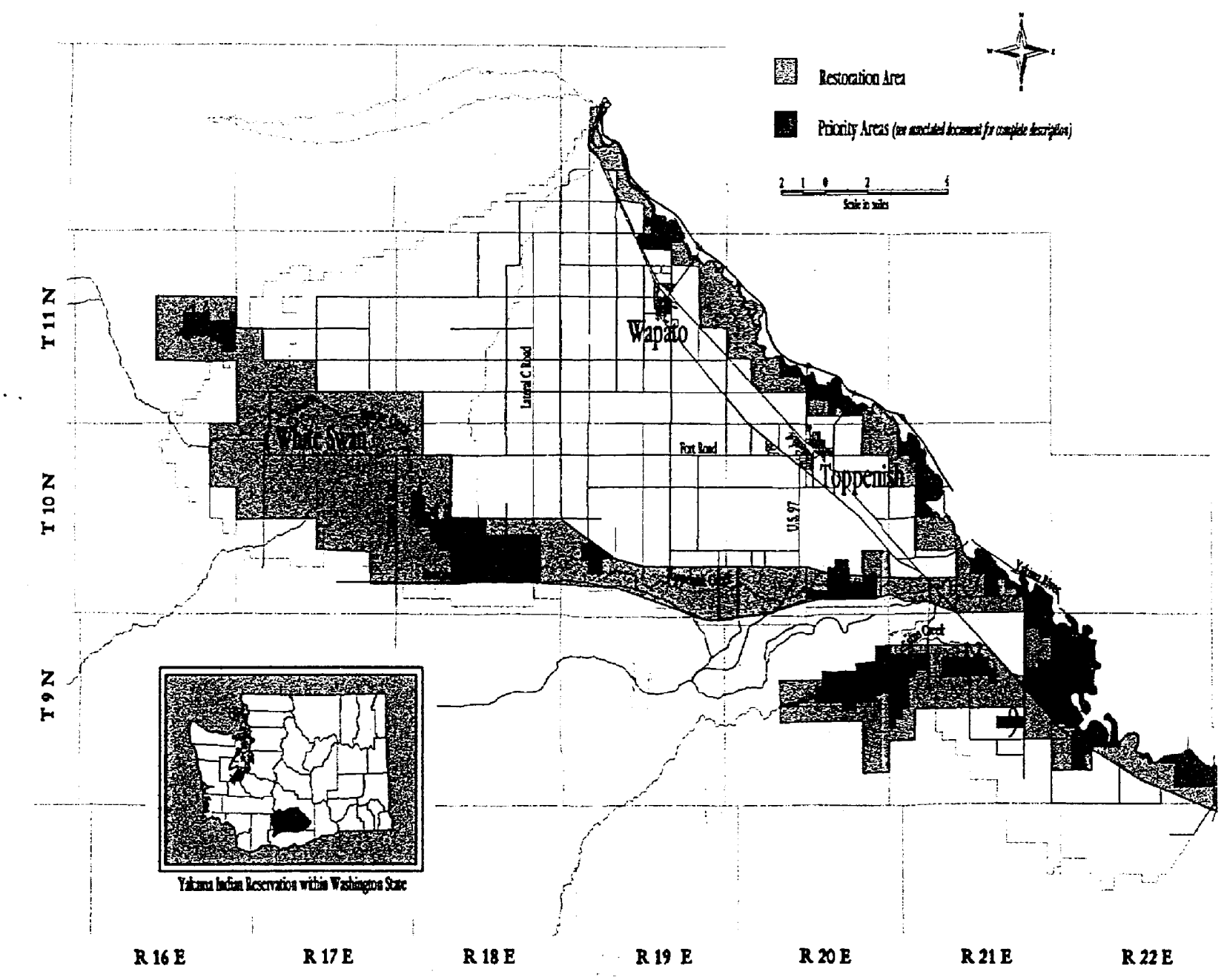

Figure 1. Regional Map, Yakama Nation Wildlife Mitigation Areas. 
Table 1. Cover-type acreages in Wildlife Management Areas, provided by the Yakama Nation and used in the 1999 Yakama Nation HEP analysis.

\begin{tabular}{|c|c|c|c|c|c|c|c|c|}
\hline & Satus & Lower Satus & Wanity Slough & $\begin{array}{l}\text { Toppenish } \\
\text { Creek }\end{array}$ & Wapato & Zimmerman & Mosebar Pond & TOTAL \\
\hline UPLAND AND RIPARIAN & $\cdot$ & & & & & & & \\
\hline Riparian Forest & 481 & 168 & 0 & 10 & 207 & 4 & 41 & 911 \\
\hline Riparian Forest - Exotic & 3 & 0 & 0 & 0 & 0 & 0 & 0 & 3 \\
\hline Riparian Herb/Forest & 9 & 0 & 0 & 0 & 0 & 0 & 0 & 9 \\
\hline Riparian Shrub & 206 & 89 & 18 & 25 & 39 & 1 & 19 & 397 \\
\hline Riparian Shrub - Exotic & 659 & 114 & 16 & 165 & 12 & 0 & 34 & 999 \\
\hline Riparian Herb & 462 & 21 & 38 & 62 & 31 & 4 & 65 & 683 \\
\hline Riparian Herb - Exotic & 0 & 10 & 0 & 4 & 0 & 0 & 44 & 58 \\
\hline Shrub Steppe - Grasslands & 1,682 & 2152 & 270 & 292 & 147 & 257 & 121 & 4,921 \\
\hline Shrub Steppe - Exotic & 0 & 100 & 0 & 37 & 47 & 0 & 0 & 184 \\
\hline Agriculture - Pasture & 146 & 954 & 8 & 368 & 223 & 1 & 63 & 1,762 \\
\hline Agriculture - Crop & $70^{\circ}$ & 0 & 00 & 135 & 0 & 0 & 0 & 205 \\
\hline \multicolumn{9}{|l|}{ WETLANDS } \\
\hline Lacustrine & 0 & 0 & 0 & 1 & 1 & 0 & 21 & 23 \\
\hline Riverine & 83 & 59 & 11 & 14 & 30 & 4 & 0 & 201 \\
\hline Riverine - Exotic & 0 & 0 & 0 & 0 & 0 & 4 & 0 & 4 \\
\hline Perennial Open Water & 285 & 0 & 0 & 0 & 0 & 0 & 0 & 285 \\
\hline Palustrine Emergent & 214 & 6 & 0 & 123 & 3 & 139 & 24 & 509 \\
\hline P. Unconsolidated Bottom & 174 & 21 & 0 & 0 & 30 & 0 & 0 & 225 \\
\hline Buildings & 0 & 0 & 0 & 0 & 0 & 20 & 0 & 20 \\
\hline TOTAL & 4,479 & 3,696 & 364 & 1,108 & 776 & 430 & 434 & 11,401 \\
\hline
\end{tabular}


Table 2. Cover-type acreages by Wildlife Management Areas used in the 1999 Yakama Nation HEP analysis.

\begin{tabular}{|c|c|c|c|c|c|c|c|c|}
\hline & Satus & $\begin{array}{l}\text { Lower } \\
\text { Satus }\end{array}$ & $\begin{array}{l}\text { Wanity } \\
\text { Slough }\end{array}$ & $\begin{array}{c}\text { Toppenish } \\
\text { Creek }\end{array}$ & Wapato & $\begin{array}{c}\text { Zimmer } \\
\text { man }\end{array}$ & $\begin{array}{l}\text { Mosebar } \\
\text { Pond }\end{array}$ & Total \\
\hline \multicolumn{9}{|l|}{$\begin{array}{l}\text { Upland and } \\
\text { Riparian }\end{array}$} \\
\hline Riparian Forest & 493 & 168 & 0 & 10 & 207 & 4 & 41 & 923 \\
\hline Riparian Shrub & 864 & 203 & 34 & 190 & 51 & 1 & 53 & 1396 \\
\hline Riparian Herb & 463 & 31 & 38 & 66 & 31 & 4 & 109 & 742 \\
\hline $\begin{array}{l}\text { Shrub-Steppe } \\
\text { Grassiand }\end{array}$ & 1682 & 2252 & 270 & 329 & 194 & 257 & 121 & 5105 \\
\hline Agriculture-pasture & 146 & 954 & 8 & 368 & 223 & 1 & 63 & 1763 \\
\hline Agriculture-crop & 70 & 0 & 0 & 135 & 0 & 0 & 0 & 205 \\
\hline \multicolumn{9}{|l|}{ Wetlands } \\
\hline Lacustrine & 0 & 0 & 0 & 1 & 1 & 0 & 21 & 23 \\
\hline Riverine & 83 & 59 & 11 & 14 & 30 & 8 & 0 & 205 \\
\hline $\begin{array}{l}\text { Palustrine Open } \\
\text { Water }\end{array}$ & 285 & 0 & 0 & 0 & 0 & 0 & 0 & 285 \\
\hline Palustrine Emergent & 214 & 6 & 0 & 123 & 3 & 139 & 24 & 509 \\
\hline $\begin{array}{l}\text { Palustrine } \\
\text { Unconsolidated } \\
\text { Bottom }\end{array}$ & 174 & 21 & 0 & 0 & 30 & 0 & $\therefore 0$ & 225 \\
\hline Buildings & 0 & 0 & 0 & 0 & 0 & 20 & 0 & 20 \\
\hline Total (Acres) & 4474 & 3694 & 361 & 1236 & 770 & 434 & 432 & 11,401 \\
\hline
\end{tabular}


Table 3. Description of cover-types used in Yakama Nation 1999 HEP analysis.

\begin{tabular}{|c|c|c|c|}
\hline Cover-type & $\begin{array}{l}\text { GIS } \\
\text { Code }\end{array}$ & $\begin{array}{l}\text { FWS } \\
\text { Code }\end{array}$ & Description \\
\hline \multicolumn{4}{|l|}{ UPLAND / RIPARIAN } \\
\hline Riparian Forest & 3 & $\mathrm{~F}$ & cottonwood and willow, $->30 \%$ tree cover \\
\hline Riparian Shrub & 1 & $\mathrm{~S}$ & willow, red osier dogwood, \\
\hline Riparian Shrub/Herb & $1 / 4$ & $\mathrm{~S} / \mathrm{H}$ & riparian shrub with at least $25 \%$ herb cover \\
\hline Riparian Herb & 4 & $\mathrm{H}$ & annual and/or perennial forbs \\
\hline Shrub-Steppe-Grassland & 9 & SSG & $\begin{array}{l}\text { sagebrush, rabbit brush, hop sage }>25 \% \text {, } \\
\text { Great Basin Rye }\end{array}$ \\
\hline Grassland & 9 & $\mathrm{G}$ & Great Basin Rye, shrubs $<25 \%$ \\
\hline Agriculture - crop & 2 & Ag-c & current or recently abandon agricultural lands \\
\hline Agriculture - crop - fallow & 2 & Ag-cf & pasture grasses \\
\hline Agriculture - pasture & 2 & Ag-p & crops - corn, beans, etc., \\
\hline Agriculture - pasture fallow & 2 & Ag-pf & weeds, pasture grasses, cheat grass \\
\hline \multicolumn{4}{|l|}{ WETLANDS } \\
\hline Lacustrine & 6 & L & perennial open water $>20$ acres, $>4$ feet deep \\
\hline Riverine & 7 & $\mathrm{R}$ & perennial flowing water \\
\hline Palustrine Open Water & & POW & $\begin{array}{l}\text { open water }<20 \text { acres, }<4 \text { feet deep in mid } \\
\text { summer }\end{array}$ \\
\hline P. Aquatic Bed & 6 & PAB4 & rooted vascular plants - yellow water lily \\
\hline P. Emergent - Persistent & 8 & PEM & bulrush, broad-leaved cattail \\
\hline P. Unconsolidated bottom & 5 & PUB & sand, gravel, cobble, mud. \\
\hline DEVELOPMENTS & & & $\vdots \vdots$ \\
\hline Buildings, Roads, Parking & & $\mathrm{U}$ & . \\
\hline
\end{tabular}


Table 4. Species - habitat (cover-type) matrix indicating the HIS models used in the HEP analysis.

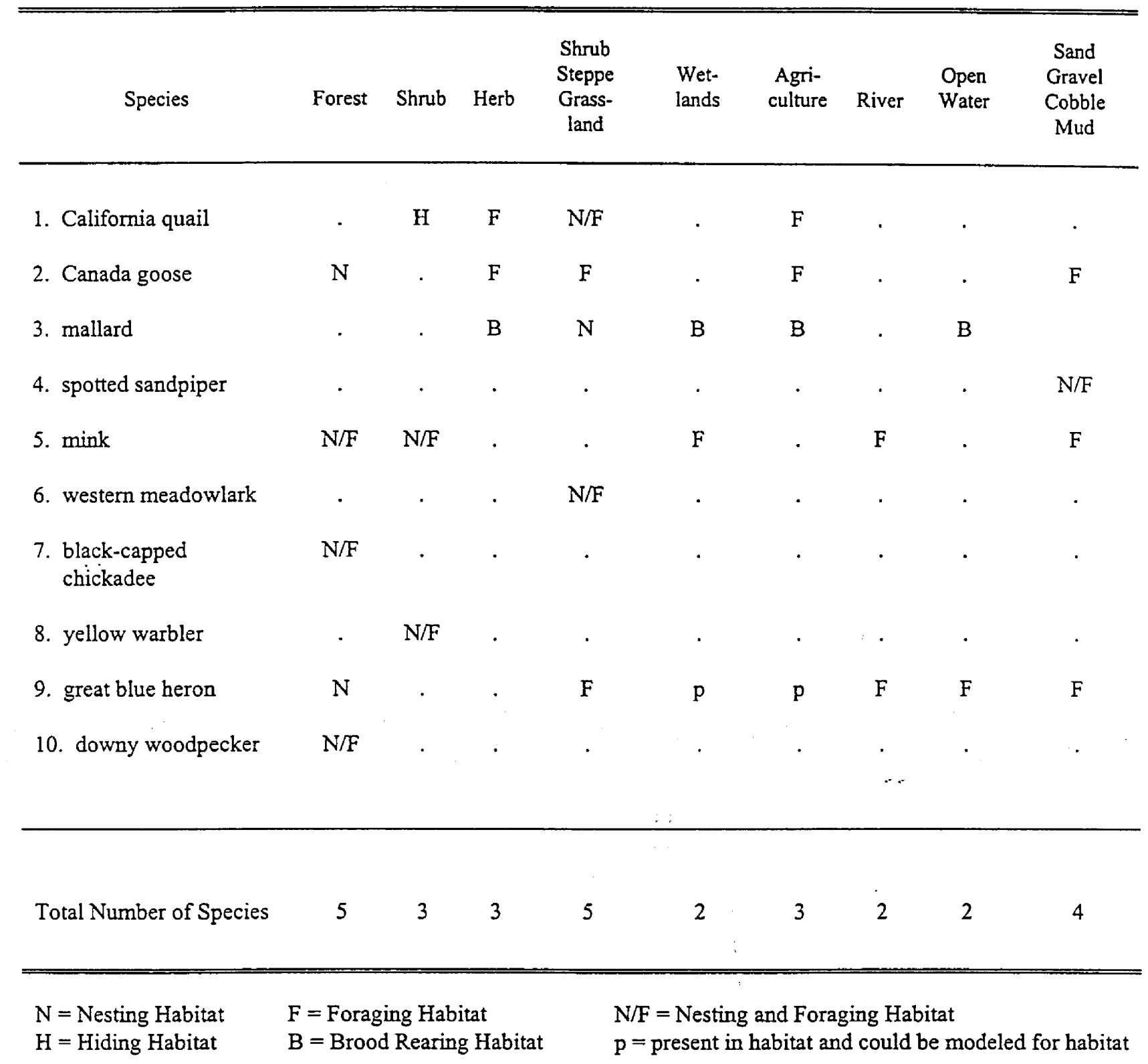


Table 5. Wildlife HEP models, Yakama Nation HEP analysis, with HSI formulas stated in EXCEL spreadsheet protocol.

\section{California Quail}
Authors:
Schroeder, Richard, USFWS
Date: 1978
Habitats:
Shrub, Pasture, Shrub-Steppe-Grass, Riparian Herb
Code:
S, Ag-p, Ag-pf, SSG, H

HSI Foraging \& Breeding $=\mathrm{V} 1+\mathrm{V} 2+(\mathrm{V} 3 \times \mathrm{V} 4 \times \mathrm{V} 5)^{\wedge} 0.333$

$\mathrm{V} 1$ - percent cover grass and herbs

$\mathrm{V} 2$ - average shrub height

V3 - distance to escape cover

V4 - diameter or width of escape cover patches

V5 - distance between escape cover patches

\section{Canada Goose}

Author: DeWard, unpublished review copy, modified to include forest cover Date: 1990

Habitats: Sand/gravel/cobble/mud, shrub-steppe-grassland, pasture, riparian herb, lacustrine, forest

Codes: $\quad$ SGCM, SSG, Ag-p, Ag-pf, H, L, POW, F

Changed in this report by RS Fleming to a nesting OR foraging model.

HSI Nesting $=\left[\mathrm{Vix}\left(\frac{\mathrm{V} 3+\mathrm{V} 4)}{2}\right]^{\wedge} 0.5\right.$

HSI Foraging $=\left(\frac{\mathrm{V} 3+\mathrm{V} 4)}{2}\right]^{\wedge} 0.5$

$\mathrm{V} 1$ - presence or absence of mature trees

V3 - distance between nesting sites and brood rearing areas

$\mathrm{V} 4$ - distance from human disturbance

\section{Mallard}

Author: Ashley, modified for Central Washington from Rasmussen and Wright, 1990 Date: 1999

Nesting Habitats: herb, grassland, shrub-steppe-grassland

Codes: H, G, SSG

HSI Nesting $\left.=\frac{[2 \mathrm{~V} 3+\mathrm{V} 4}{3} \times \mathrm{V} 5\right]^{\wedge} 0.5 \times \mathrm{V} 6$

Brood Rearing Habitats: emergent wetlands, lacustrine, palustrine emergent

Code: H, G, SSG, PEM, PAB4, POW, L

HSI Brood Rearing $=$ V7 $\times$ V8 $\times$ V9 
Table 5. Continued.

V3 - herb and shrub percent cover within 300 feet of shoreline

V4 - herb and shrub percent cover from 300 to 600 feet from shoreline

V5 - height of nesting cover

V6 - distance from human disturbance

V7 - ration of emergent vegetation to open water

V8 - distance between nesting and brood rearing areas

V9 - water regime

\section{Spotted Sandpiper}

Author: Dorsey, G.L., unpublished FWS review copy $\quad$ Date: 1990

Habitats: Sand-gravel-cobble-mud

Codes: $\quad$ SGCM

HSI Foraging \& Breeding $=\frac{\mathrm{V} 1+\mathrm{V} 2+\mathrm{V} 3}{3}$

$\mathrm{V} 1$ - percent cover of herbs less than 2 feet high

$\mathrm{V} 2$ - distance from nesting sites to open water or river

V3 - percent cover of organic debris

\section{Mink}

Author: Allen, A.W. Date: 1986

Habitats: Riverine, emergent, forest, shrub, sand-gravel-cobble-mud

Code: $\quad$ R, PEM, PAB4, F, S, SGCM

HSI Foraging - Forest and Shrub $=\operatorname{Min}\left(1.0, \frac{\mathrm{V} 2+\mathrm{V} 3+\mathrm{V} 4}{2}+\mathrm{V} 5\right.$

HSI Foraging - Emergent Wetlands
(PEM, PAB4)

(PEM, PAB4)

HSI Foraging - Riverine and SGCM $=(\mathrm{V} 5 \times \mathrm{V} 6)^{\wedge} 0.5$

V2 - percent cover of tree canopy

V3 - percent cover of shrub canopy

$\mathrm{V} 4$ - percent cover to emergent vegetation

V5 - percent tree cover within 330 feet of the shoreline

V6 - percent cover of trees and shrubs along the shoreline

\section{Western Meadow Lark}

Author: Schroeder \& Sousa, modified

Date: 1982

Habitats: Shrub-steppe-grassland and pasture

Codes: $\quad$ SSG, Ag-p, Ag-pf, G

HSI Foraging \& Breeding $=(\mathrm{V} 1 \times \mathrm{V} 2 \times \mathrm{V} 3 \times \mathrm{V} 4)^{\wedge} 0.5 \times \mathrm{V} 5$ 
Table 5. Continued.

$\mathrm{V} 1$ - percent cover of herbs and grass

V2 - percent cover of grass

V3 - average height of her and grass cover

V4 - distance to perch sites

V5 - percent shrub cover

\section{Black-capped Chickadee}
Author:
Schroeder
Date: $1983 a$
Habitats:
Forest
Code:
$\mathrm{F}$

HSI Foraging \& Breeding = lowest value for V1, V2, V3

In practice $=\mathrm{V} 1 \times \mathrm{V} 2 \times \mathrm{V} 3$ does not to exceed 1.0

V1 - percent tree cover

V2 - average height of trees

V3 - number of snags 4 to 10 inches in diameter per acre

\section{Yellow Warbler}
Author: Schroeder
Date: 1982
Habitats: Shrub
Codes: $\quad S$

HSI Foraging \& Breeding $=(\mathrm{V} 1 \times \mathrm{V} 2 \times \mathrm{V} 3)^{\wedge} 0.333$

V1 - percent cover of deciduous shrubs

V2 - average height of deciduous shrubs

V3 - percent cover of hydrophytic shrubs

\section{Great Blue Heron}

Author: Short and Cooper, modified to include forest nesting Date: 1985

Habitats: Nesting: forest

Code: $F$

Foraging: sand-gravel-cobble-mud, shrub-steppe-grassland, riverine, lacustrine, pasture

Code: SGCM, SSG, R, L, POW, Ag-p, Ag-pf

HSI - Nesting $=(\mathrm{V} 1 \times \mathrm{V} 2 \times \mathrm{V} 3 \times \mathrm{V} 4 \times \mathrm{V} 5 \times \mathrm{V} 6)^{\wedge} 0.5$

HSI - Foraging (all other cover types) $=(\mathrm{V} 1 \times \mathrm{V} 2 \times \mathrm{V} 3)$ 
Table 5. Continued.

V1 - distance between nesting and feeding sites

V2 - forage quality

V3 - distance to human disturbance

V4 - availability of nesting sites

V5 - disturbance at nesting sites

V6 - actual and potential distance to nesting sites from foraging areas

\section{Downy Woodpecker}
Author: Schroeder
Date: $\quad 1983 b$
Habitats: Mature forest
Codes: $\quad F$

HSI Foraging \& Breeding = lowest value V1 or V2

In practice $\mathrm{V} 1 \mathrm{x} \mathrm{V} 2$ seldom exceeds 1.0 . In the cases where it exceeds 1.0 , the value was reset to 1.0 .

$\mathrm{V} 1$ - basal area per acre

V2 - number of snags from 7 to 9 inches in diameter per acre 


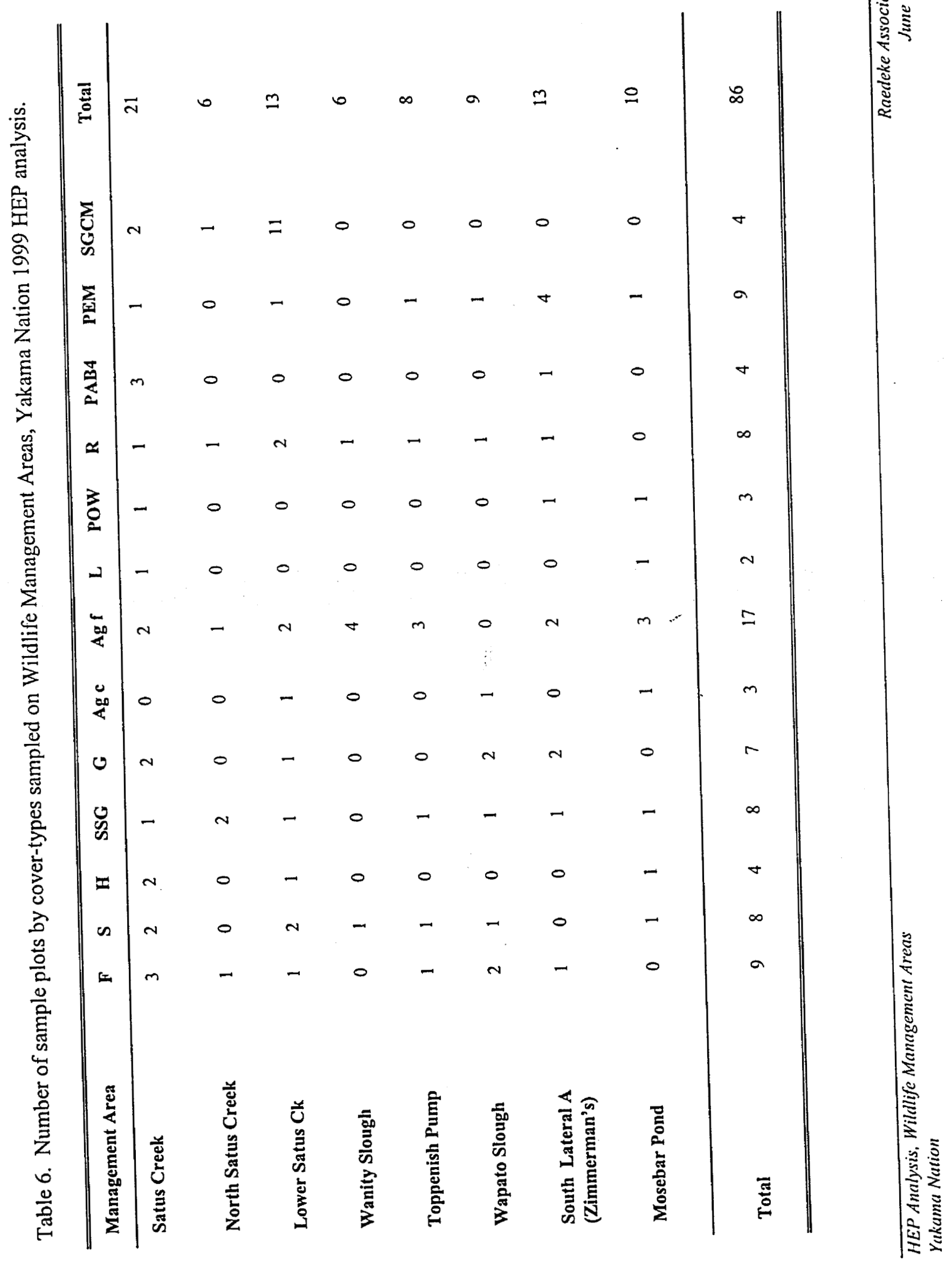




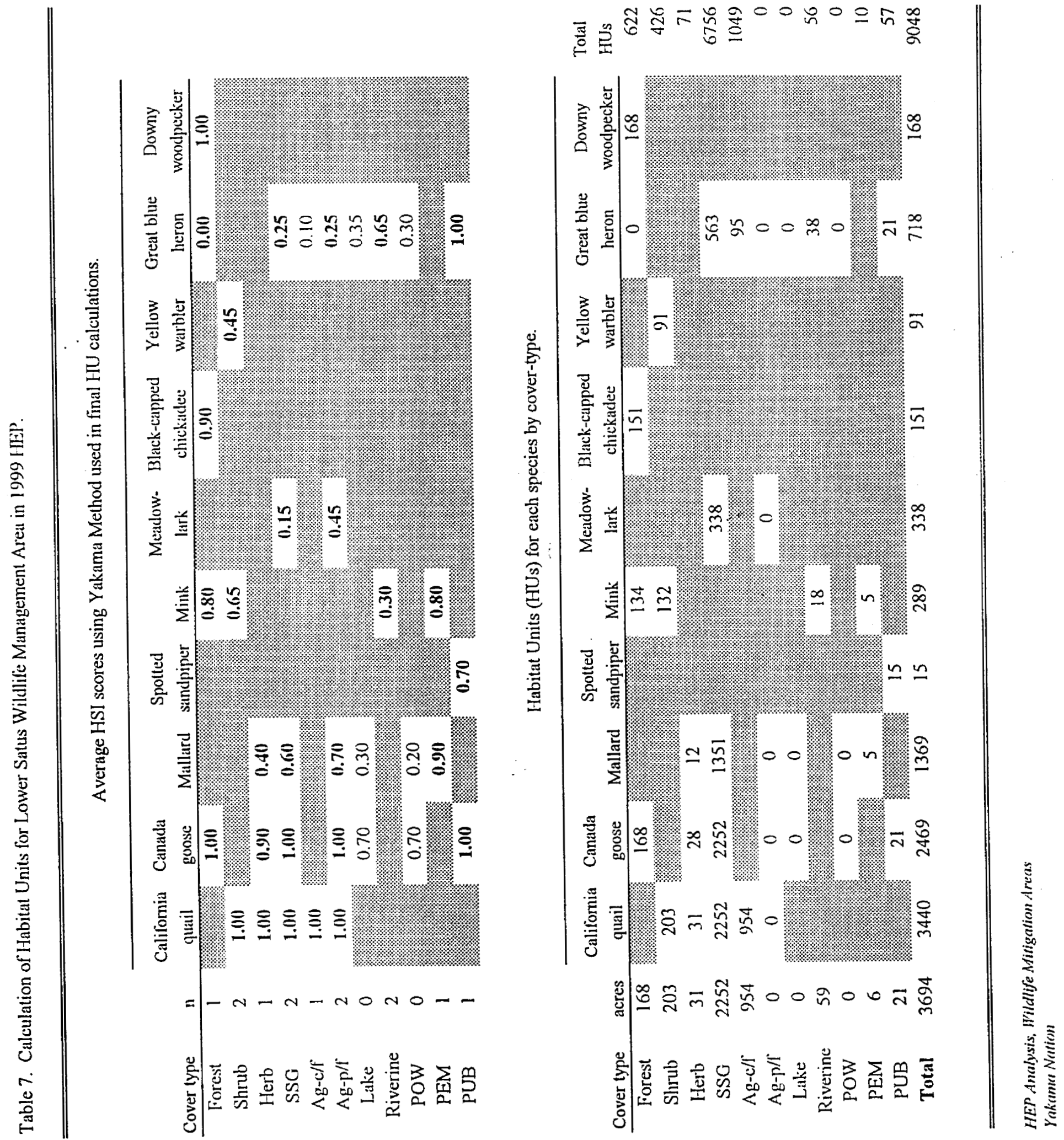




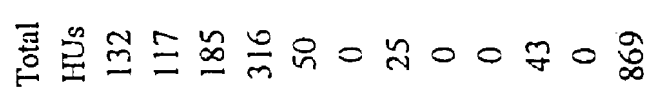
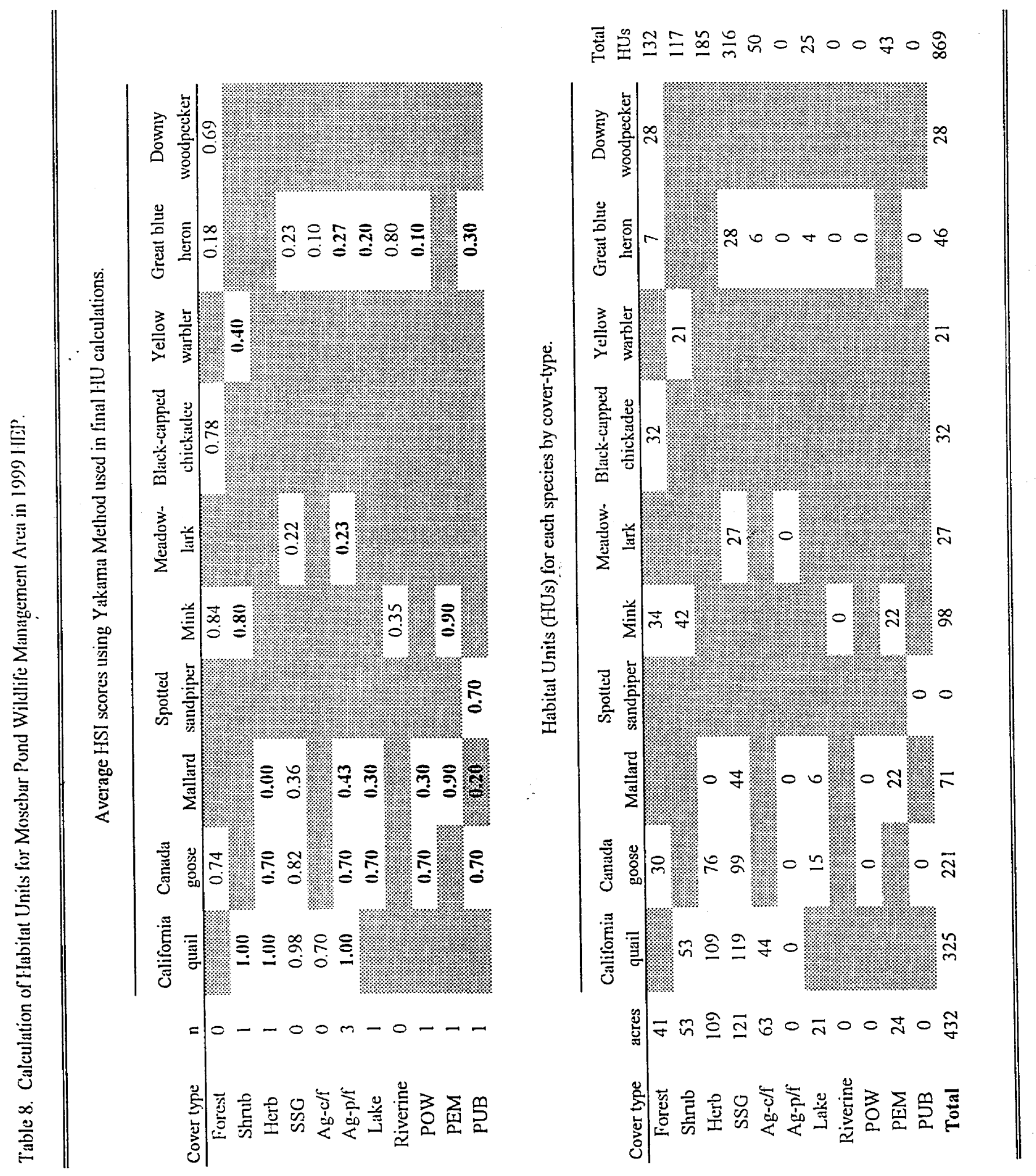

言

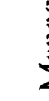

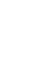

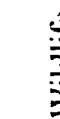

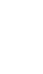




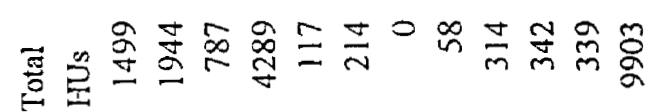
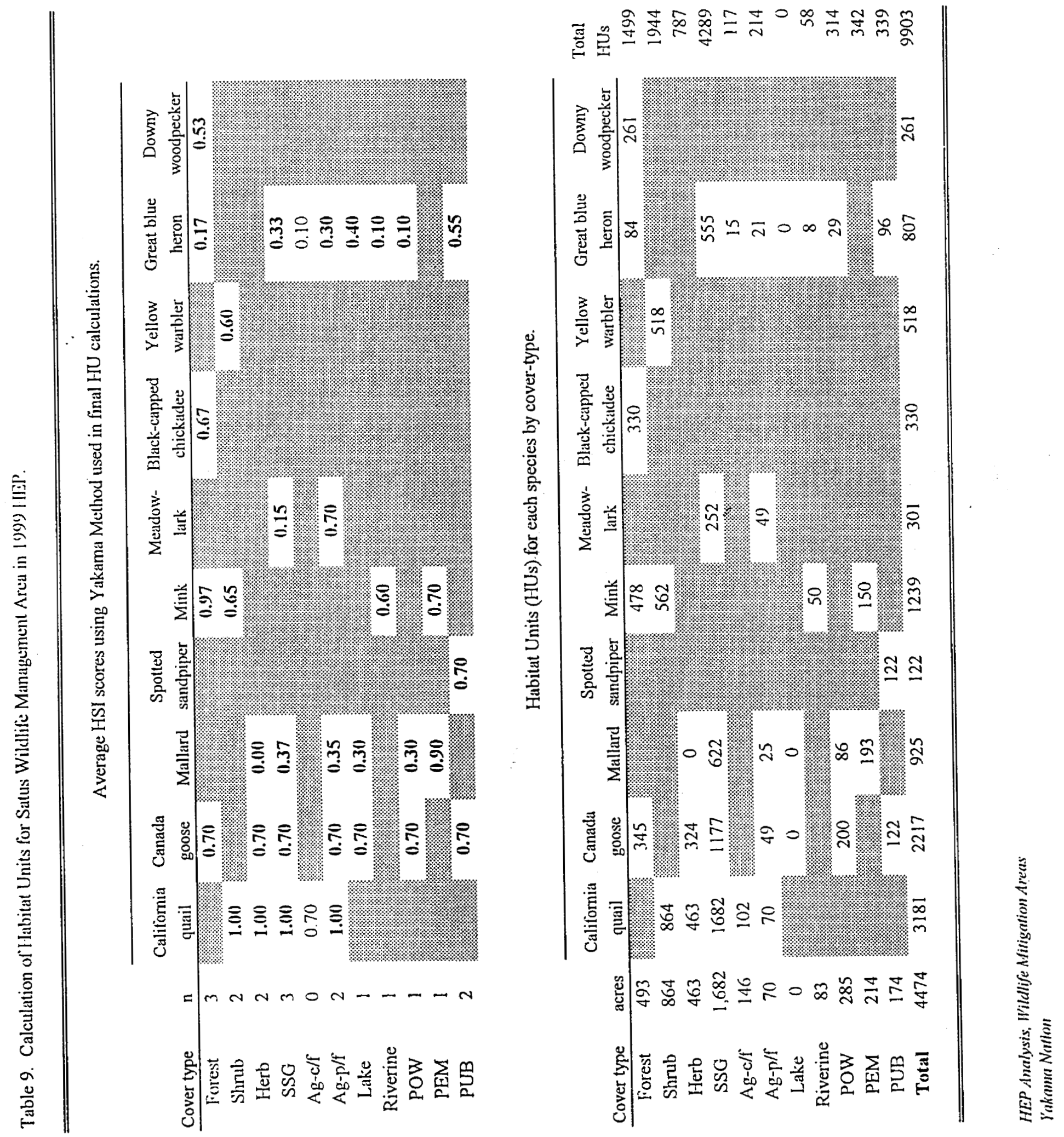

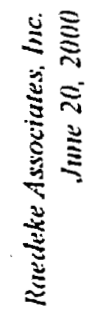



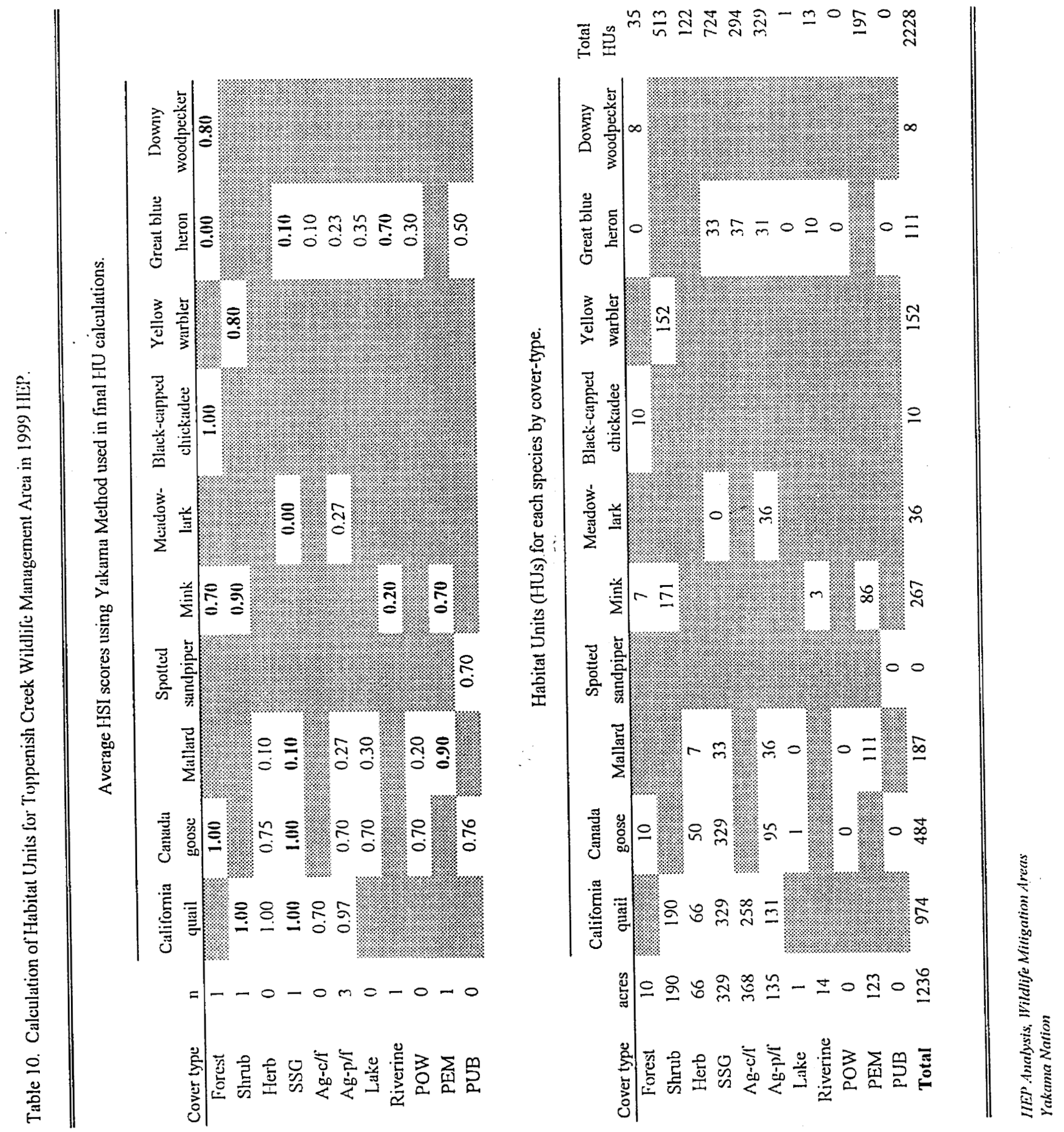


\begin{tabular}{|c|c|c|c|c|c|c|c|c|c|c|c|}
\hline \multirow[b]{2}{*}{ Cover type } & \multirow[b]{2}{*}{$\mathbf{n}$} & \multicolumn{10}{|c|}{ Average HSI scores using Yakama Method used in final IVU calculations. } \\
\hline & & $\begin{array}{l}\text { Culifornia } \\
\text { quail }\end{array}$ & $\begin{array}{l}\text { Cantada } \\
\text { goose }\end{array}$ & Mallard & $\begin{array}{c}\text { Spotted } \\
\text { sandpiper }\end{array}$ & Mink & $\begin{array}{c}\text { Meadow- } \\
\text { lark }\end{array}$ & $\begin{array}{c}\text { Black-ciapped } \\
\text { chickadee }\end{array}$ & $\begin{array}{l}\text { Yellow } \\
\text { warbler }\end{array}$ & $\begin{array}{c}\text { Greal blue } \\
\text { heron }\end{array}$ & $\begin{array}{c}\text { Downy } \\
\text { woodpecker }\end{array}$ \\
\hline Forest & 1 & ר. & 1.00 & & & 0.80 & & 0.90 & & 0.00 & 1.00 \\
\hline Shrub & 2 & 1.00 & & & & 0.65 & & & 0.45 & & \\
\hline Herb & 1 & 1.00 & 0.90 & 0.40 & & & & & & & \\
\hline SSG & 2 & 1.00 & 1.00 & 0.50 & & & 0.15 & & & 0.25 & \\
\hline$\wedge \mathrm{g}-\mathrm{c} / \mathrm{f}$ & 1 & 1.00 & & & & & & & & 0.10 & \\
\hline $\mathrm{Ag}-\mathrm{p} / \mathrm{I}$ & 2 & 1.00 & 1.00 & 0.70 & & & 0.45 & & & 0.25 & \\
\hline Lake & 0 & & 0.70 & 0.30 & & & & & & 0.35 & \\
\hline Riverine & 2 & & & & & 0.30 & & & & 0.65 & \\
\hline POW & 0 & & 0.70 & 0.20 & & & & & & 0.30 & \\
\hline PEM & 1 & & & 0.90 & & 0.80 & & & & & \\
\hline PUB & 5 & & 1.00 & ; & 0.70 & & & & & 1.00 & \\
\hline
\end{tabular}

Habitat Units (HUs). for each species by cover-type.

\begin{tabular}{|c|c|c|c|c|c|c|c|c|c|c|c|c|}
\hline Cover type & acres & $\begin{array}{l}\text { California } \\
\text { quail }\end{array}$ & $\begin{array}{c}\text { Canada } \\
\text { goose }\end{array}$ & Mallard & $\begin{array}{c}\text { Spotted } \\
\text { sandpiper }\end{array}$ & Mink & $\begin{array}{c}\text { Meadow- } \\
\text { lark }\end{array}$ & $\begin{array}{c}\text { Black-capped } \\
\text { chickadee }\end{array}$ & $\begin{array}{l}\text { Yellow } \\
\text { warbler }\end{array}$ & $\begin{array}{c}\text { Great blue } \\
\text { heron }\end{array}$ & $\begin{array}{c}\text { Downy } \\
\text { woodpecker }\end{array}$ & $\begin{array}{l}\text { Total } \\
\text { HUs }\end{array}$ \\
\hline Forest & 0 & & 0 & & & 0 & \% & 0 & & 0 & 0 & 0 \\
\hline Shrub & 34 & 34 & & & & 22 & & & 15 & 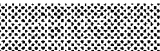 & & 71 \\
\hline Herb & 38 & 38 & 34 & 15 & & & & & & & & 87 \\
\hline SSG & 270 & 270 & 270 & 135 & & & 41 & & & 68 & & 783 \\
\hline$\Lambda g-c / s$ & 8 & 8 & : & $\%$ & & & ষ & & & 1 & & 9 \\
\hline Ag-p/f & 0 & 0 & 0 & 0 & & & 0 & & & 0 & & 0 \\
\hline Lake & 0 & & 0 & 0 & & & & & & 0 & & 0 \\
\hline Riverine & 11 & & & & & 3 & & & & 7 & & 10 \\
\hline POW & 0 & & 0 & 0 & & & & & & 0 & & 0 \\
\hline PEM & 0 & & & 0 & & 0 & & & & 2 & & 0 \\
\hline PUB & 0 & & 0 & ;: & 0 & & & & & 0 & & 0 \\
\hline Total & 361 & 350 & 304 & 150 & 0 & 25 & 41 & 0 & 15 & 75 & 0 & 961 \\
\hline
\end{tabular}




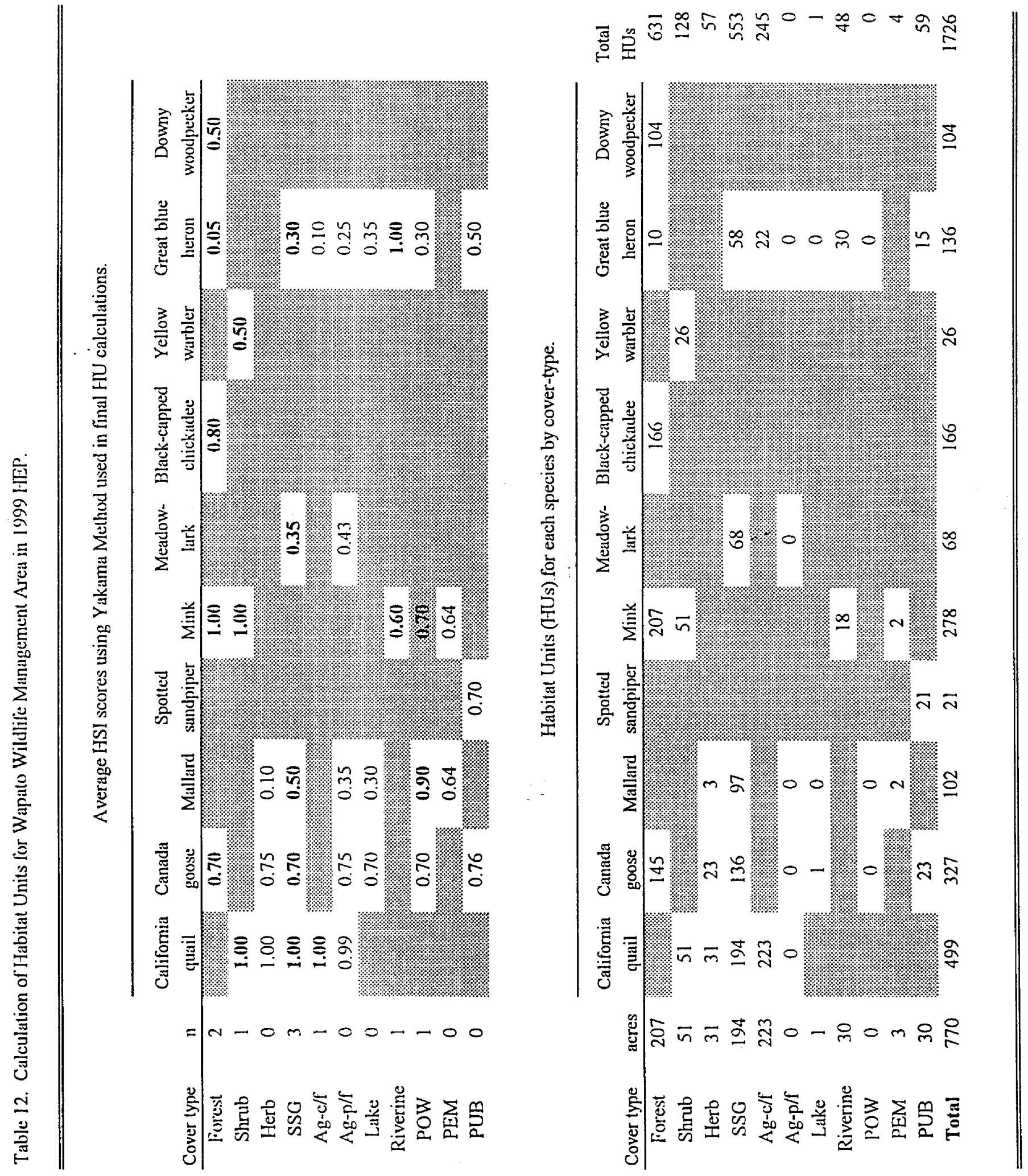




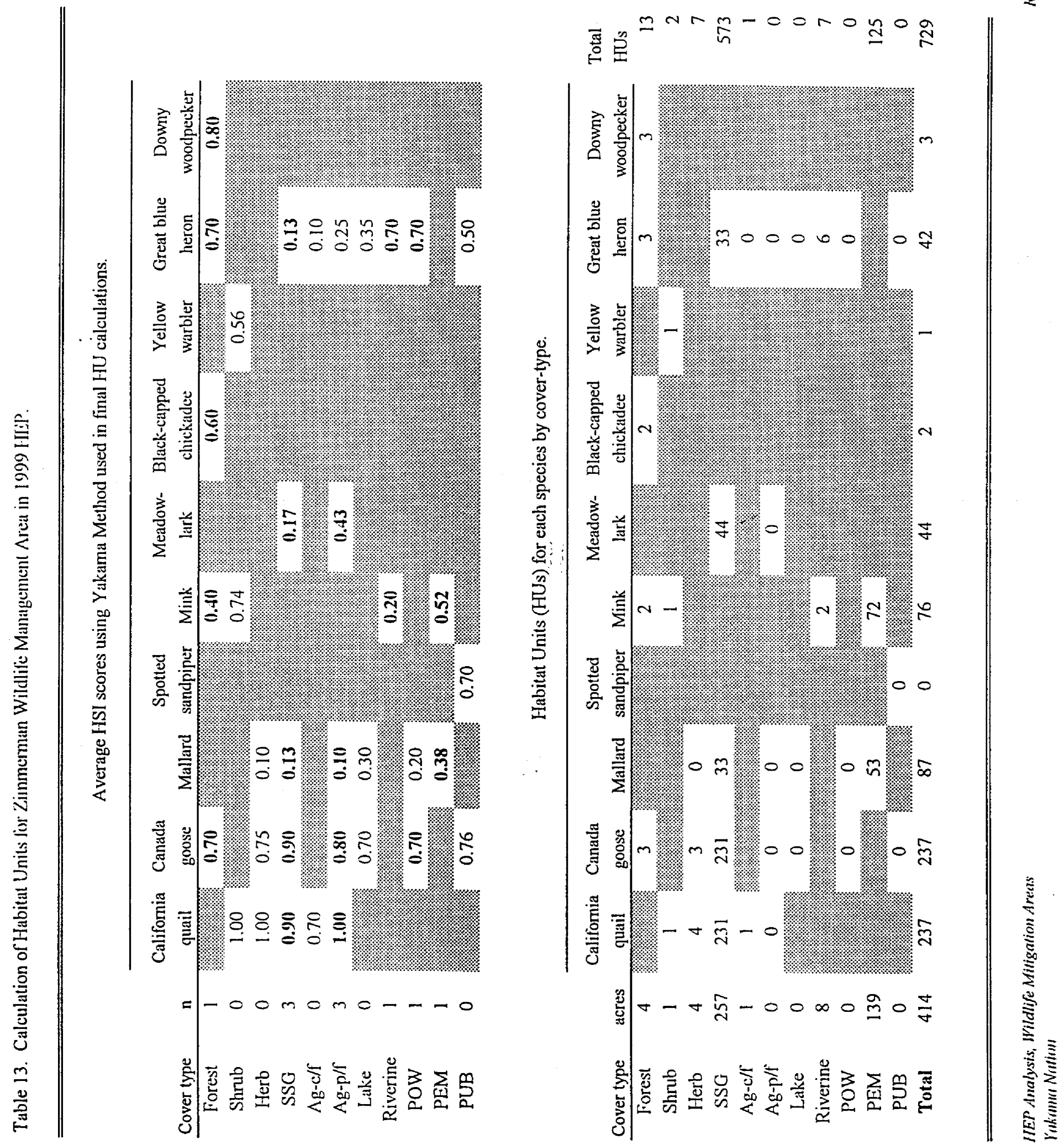




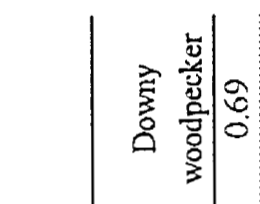

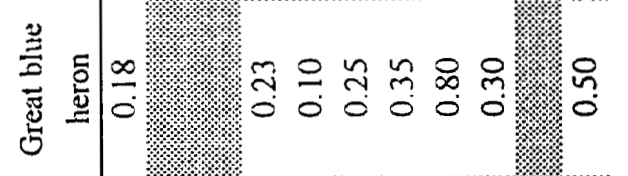

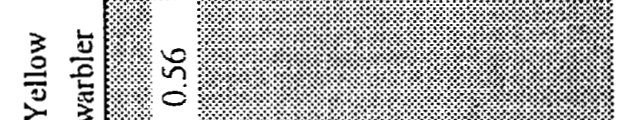

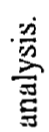

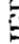

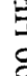

g

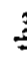

.

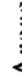

(1)

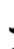

.

.

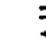
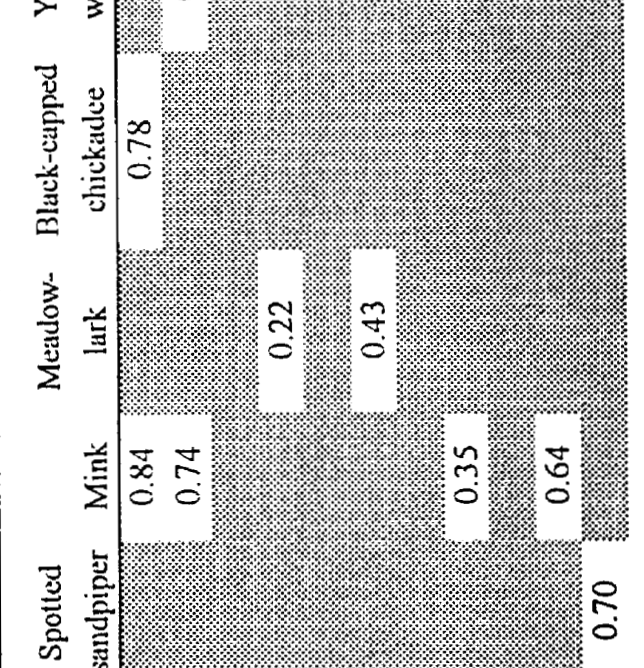


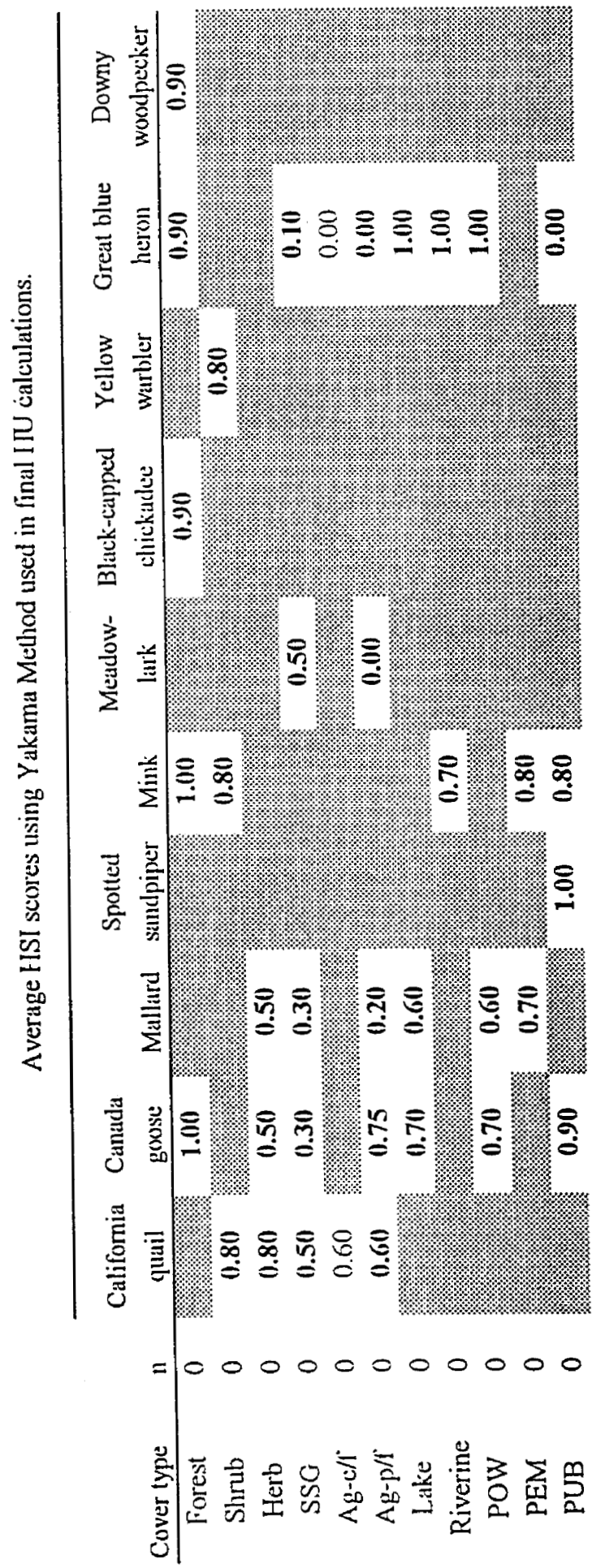

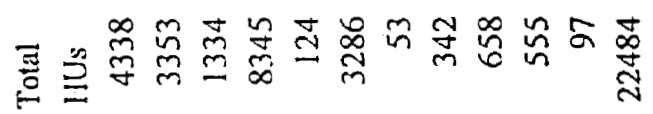

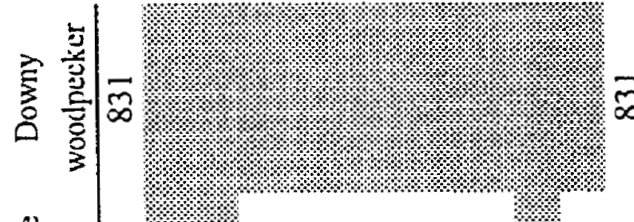

焉

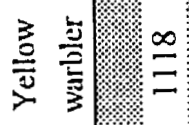

$\stackrel{\infty}{=}$

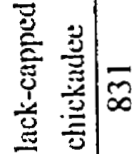

$\bar{\infty}$

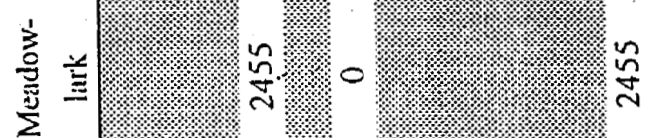

总

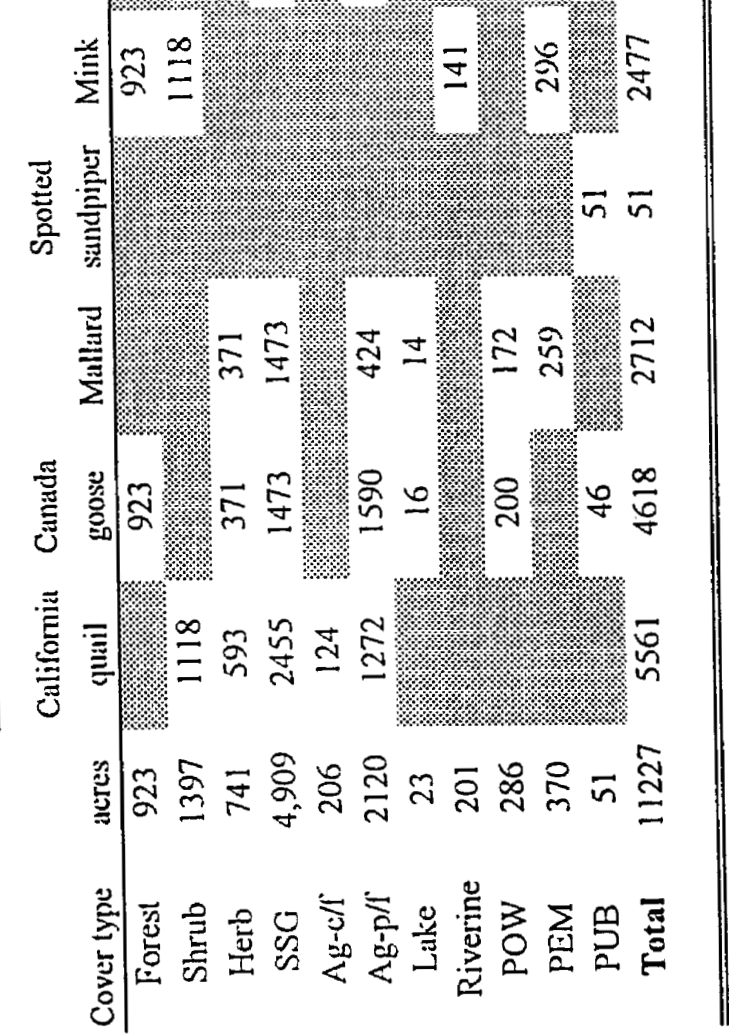


Table 16. Cover-types and acreages used in the 1990 and 1999 Yakama Nation HEP Analyses.

\begin{tabular}{|c|c|c|c|c|c|}
\hline $\begin{array}{l}\text { Cover-Types } \\
\text { Description }\end{array}$ & $\begin{array}{c}\text { YN } \\
\text { GIS Code }\end{array}$ & $\begin{array}{l}\text { Code } \\
\text { in this } \\
\text { Report }\end{array}$ & $\begin{array}{l}1990 \text { Project } \\
\text { Area (acres) }\end{array}$ & $\begin{array}{l}1999 \text { Project } \\
\text { Area (acres) }\end{array}$ & $\begin{array}{l}\text { Percent } \\
\text { Change }\end{array}$ \\
\hline \multicolumn{6}{|l|}{ UPLANDS } \\
\hline Riparian Forest & 3 & F & 887 & 911 & 102.7 \\
\hline Riparian Forest - exotics & 3 & $\mathrm{Fe}$ & 27 & 3 & 11.1 \\
\hline Riparian Forest - Herb & $4 / 3$ & $\mathrm{~F} / \mathrm{H}$ & 9 & 9 & 0.0 \\
\hline Riparian Shrub & 1 & $S$ & 397 & 397 & 0.0 \\
\hline Riparian Shrub - exotics & 1 & $\mathrm{Se}$ & 999 & 999 & 0.0 \\
\hline Riparian Shrub - Herb & $4 / 1$ & $\mathrm{~S} / \mathrm{H}$ & 1 & & \\
\hline Riparian Herb & 4 & $\mathrm{H}$ & 683 & 683 & 0.0 \\
\hline Riparian Herb - exotics & 4 & $\mathrm{He}$ & 58 & 58 & 0.0 \\
\hline Shrub-Steppe-Grass & 9 & SSG & 4725 & 4921 & 104.1 \\
\hline Shrub-Steppe - exotics & 9 & SSGe & 184 & 184 & 0.0 \\
\hline Agriculture - crop & 2 & $\mathrm{Ag}-\mathrm{C}$ & 206 & 205 & 99.5 \\
\hline Agriculture - pasture & 2 & Ag-p & 2120 & 1763 & 83.2 \\
\hline WETLANDS & & & & \multicolumn{2}{|l|}{$\cdots$} \\
\hline Lacustrine- Pond & 6 & $L-P$ & 23 & 23 & 0.0 \\
\hline Palustrine Open Water & 6 & POW & 286 & 285 & 99.6 \\
\hline Riverine & 7 & $\mathrm{R}$ & 0201 & 201 & 0.0 \\
\hline Riverine - exotic & 7 & $\operatorname{Re}$ & 0 & 4 & \\
\hline Emergent & 8 & PEM & 156 & 295 & 189.1 \\
\hline Emergent - exotics & 8 & PEMe & 214 & 214 & 0.0 \\
\hline Sand Gravel Cobble Mud & 5 & PUB & 51 & 225 & 441.2 \\
\hline \multicolumn{6}{|l|}{ OTHER } \\
\hline Buildings - Developments & & $\mathrm{D}$ & 20 & 20 & 0.0 \\
\hline Total & & & 11,247 & 11,401 & 101.4 \\
\hline
\end{tabular}




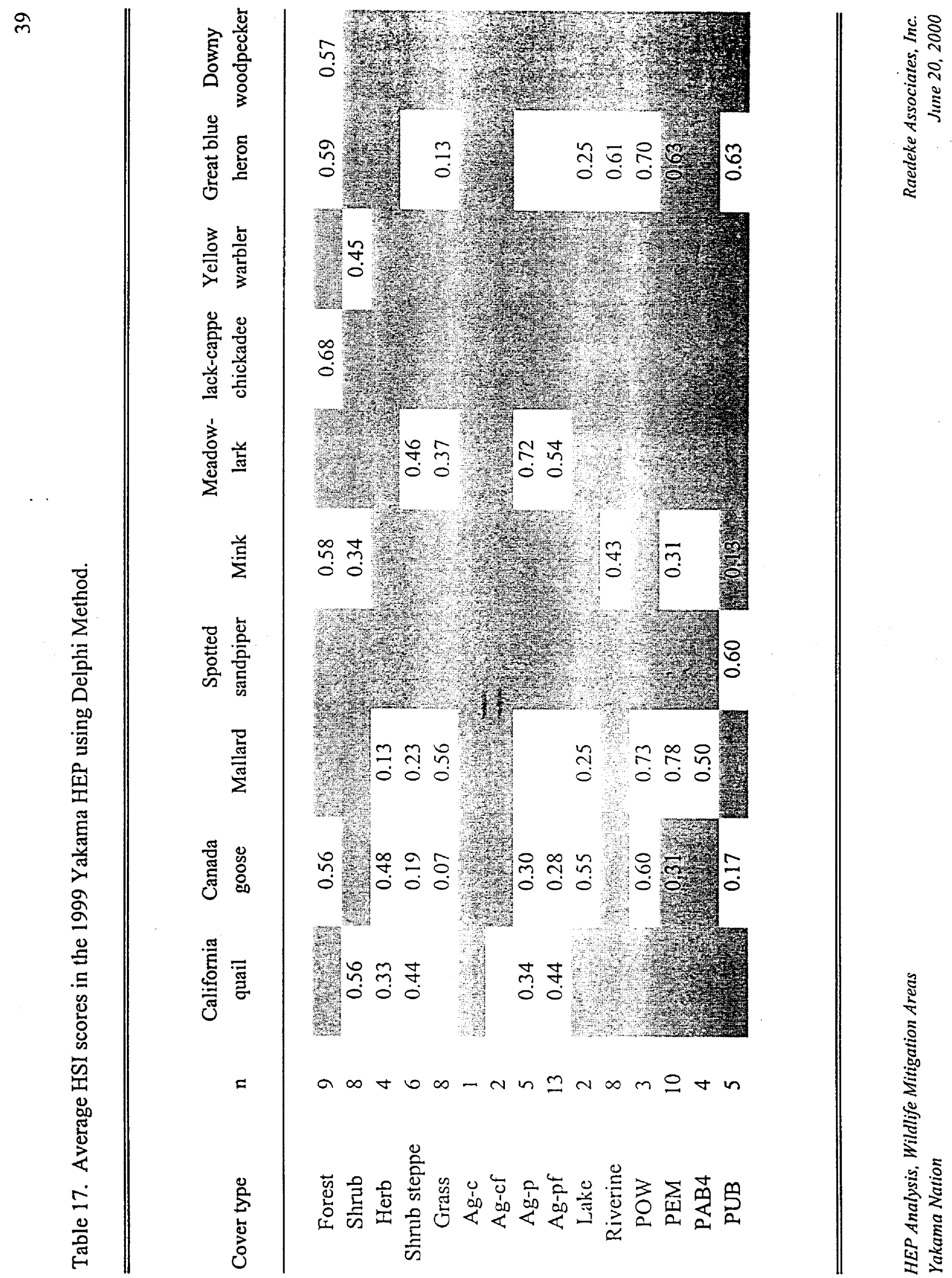




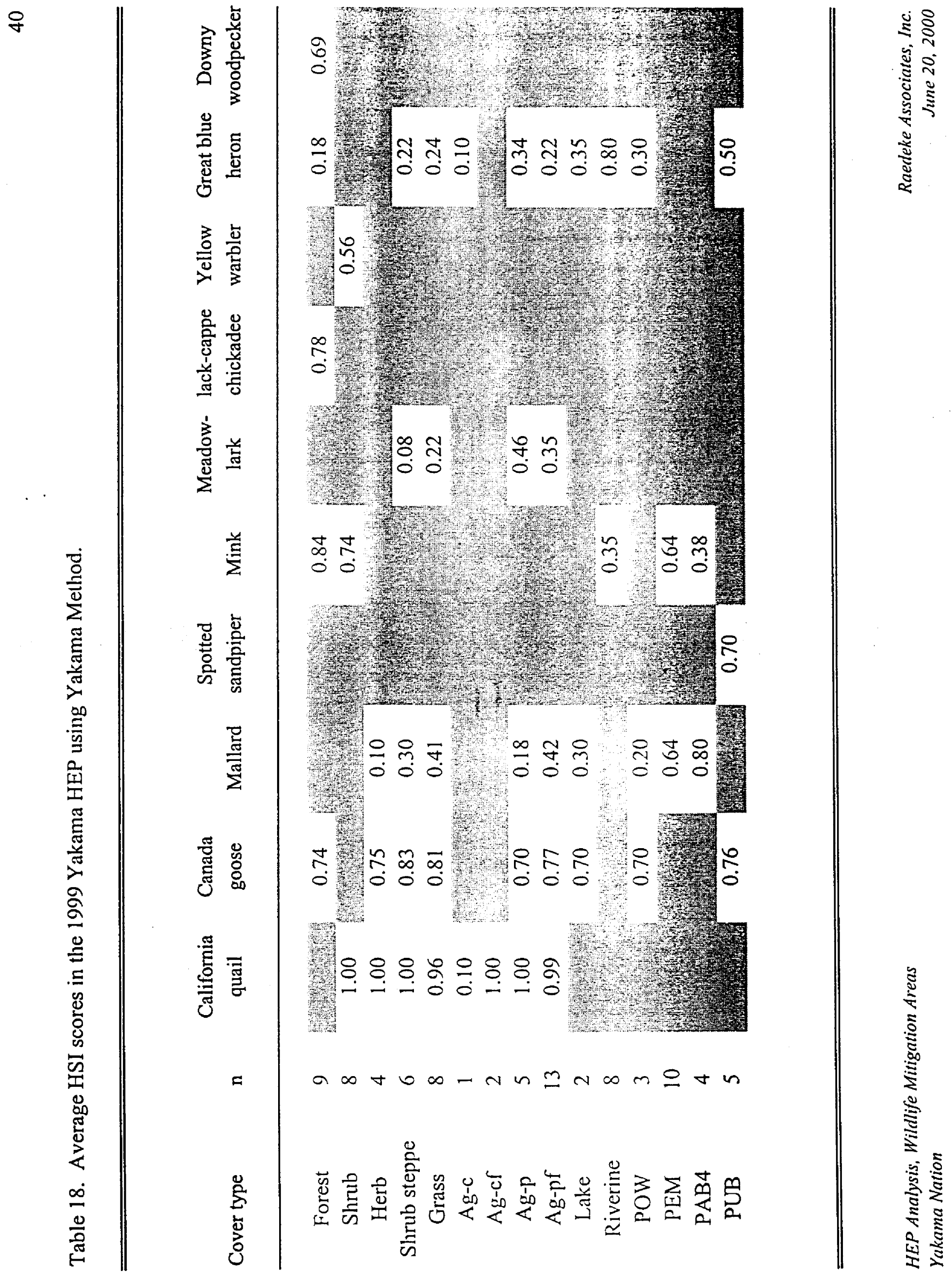


Table 19. Comparison of overall HSI values from the Delphi and Yakama Methods as part of the 1999 HEP assessment. Tabled values are overall averages by species.

\begin{tabular}{lccc}
\hline & $\begin{array}{c}\text { Sample } \\
\text { size }\end{array}$ & Delphi & Yakama \\
Species & 5 & 0.42 & 1.00 \\
\hline California quail & 9 & 0.36 & 0.72 \\
Canada goose & 7 & 0.45 & 0.46 \\
Mallard & 1 & 0.60 & 0.70 \\
Spotted sandpiper & 4 & 0.42 & 0.64 \\
Mink & 4 & 0.52 & 0.29 \\
Western Meadowlark & 1 & 0.68 & 0.78 \\
Black-capped chickadee & 1 & 0.45 & 0.56 \\
Yellow warbler & 5 & 0.44 & 0.40 \\
Great blue heron & 1 & 0.57 & 0.70 \\
Downy woodpecker & & & \\
\hline & 38 & 0.44 & 0.61 \\
Total & & & \\
\hline
\end{tabular}


Table 20. Comparison of effort required for HEP analysis using the Delphi, Yakama and Transect methodologies. Tabled values are effort required to sample 100 plots.

\begin{tabular}{lllll} 
& & & & \\
Phase/Task & Delphi & Yakama & Transect & Notes \\
\hline
\end{tabular}

Field Preparation

$\begin{array}{rcccc}\text { Scoping } & 5 & 5 & 5 & \\ \text { Species selection } & 1 & 1 & 1 & 1 \\ \text { Habitat mapping } & & 10 & 10 & 2 \\ \text { Model building } & 4 & 2 & 2 & \end{array}$

\section{Field Sampling}

Travel time

21

16

40

Data collection

12

6

222

3

\section{Computation Tasks}

Create spreadsheets

Data analysis

Data summary

0
1
1

$\begin{array}{ll}5 & 5 \\ 4 & 4 \\ 1 & 1\end{array}$

Total Effort

47

50

290

Notes:

1 Would be the same for all methods

2 We assume 10 species, with 5 new models and 5 models unaltered

3 Values are for a three-person team 


\section{APPENDIX A}

Sampling Plan 
Table A-1. Participants, Yakama Nation HEP Analysis, August 1999.

\section{Paul ASHLEY}

Washington Department of Fish and Wildlife

Brian M. AULD

Natural Resources Division, Kalispel Tribe

Susan P. BARNS

Oregon Department of Fish and Wildlife

Jeff FEEN

Wildlife Resource Management, Yakama Nation

Richard S. FLEMING

Raedeke Associates, Inc.

Henry FRASER

Wildlife Resource Management, Yakama Nation

Tracy HAMES

Wildlife Resource Management, Yakama Nation

\section{Darren HOLMES}

Natural Resources Department, Kalispel Tribe

Laurel JAMES

Wildlife Resource Management, Yakama Nation

Gina KING

Wildlife Resource Management, Yakama Nation

Don LARSEN

Wildlife Resource Management, Yakama Nation

\section{Steven LEWIS}

Columbia National Wildlife Refuge, US Fish and Wildlife Service

David NUNN

South Yakima Conservation District

Brooke PALMER

Columbia National Wildlife Refuge, US Fish and Wildlife Service

Jim STEVENSON

Wildlife Resource Management, Yakama Nation 
Bill STEWART

US Fish and Wildlife Service

Theodora STRONG

Wildlife Resource Management, Yakama Nation

Haace St. MARTIN

Burns Paiute Tribe

Richard TEMPLE 
Table A-2. Participation, Yakama Nation HEP Analysis, August 1999.

\begin{tabular}{|c|c|c|c|c|c|c|c|c|c|c|}
\hline August & 6 & 9 & 10 & 11 & 12 & 13 & 17 & 18 & 19 & $20^{*}$ \\
\hline Ashley & $X$ & & & $X$ & $\mathrm{X}$ & & & & & \\
\hline Auld & & $\mathrm{X}$ & $\mathrm{X}$ & & & & & $\mathrm{X}$ & $\mathrm{X}$ & \\
\hline Barns & & & & & & & & $\mathrm{X}$ & & \\
\hline Feen & & & & & $\mathrm{X}$ & & & & & \\
\hline Fleming & $\mathrm{X}$ & $X$ & $\mathrm{X}$ & $X$ & $X$ & $\mathrm{X}$ & $X$ & $X$ & $X$ & $X$ \\
\hline Fraser & & $\mathrm{X}$ & & & $\mathrm{X}$ & & & & & \\
\hline Hames & $\mathrm{X}$ & $\mathrm{X}$ & $X$ & $\mathrm{X}$ & $\mathrm{X}$ & $X$ & $X$ & $X$ & $\mathrm{X}$ & $\mathrm{X}$ \\
\hline Holmes & & $\mathrm{X}$ & $X$ & & & & & $X$ & $\mathrm{X}$ & \\
\hline James & & & & $\mathrm{X}$ & $X$ & $X$ & & & & \\
\hline King & & & & $X$ & & & & & & \\
\hline Larsen & & & & & & & & & $X$ & \\
\hline Lewis & & & $X$ & $\mathrm{X}$ & & & & & & \\
\hline Nụnn & & & & $X$ & $\mathrm{X}$ & & & & & \\
\hline Palmer & & & $X$ & $\mathrm{X}$ & & & & & & \\
\hline Stevenson & & $X$ & & & $\mathrm{X}$ & & & & & \\
\hline Stewart & & & & $\mathrm{X}$ & & & & & & \\
\hline Strong & & & & & & & & & $\mathrm{X}$ & \\
\hline St. Martin & & & & $\mathrm{X}$ & $X$ & & & & & \\
\hline Temple & & $X$ & & & & & & & & \\
\hline TOTAL & 3 & 7 & 6 & 10 & 9 & 3 & 2 & 5 & 7 & 2 \\
\hline
\end{tabular}

* Office 
APPENDIX B

Delphi Method 


\section{B-1}

Table B-1. Delphi Word Models for Species, Yakama Nation HEP Analysis.

The word models below have been adapted for use in the evaluation of lands in the Yakima River Valley near Toppenish, Washington. Some of these lands remain near natural riparian forests and wetlands. Other lands have been in irrigation agriculture for many years. The Yakama Nation is in the process of restoring the agricultural lands as wildlife habitat. The descriptions below are guides for assigning a single habitat rating (HSI) for each species at each sample site. In this exercise, the intention is a quick evaluation of the habitat at a sample station or plot. The plot would be approximately one acre in area (a radius of 118 feet) centered, or off centered in some cases, upon the designated station.

The description for the HSI values provide reference points. Values assigned in the filed can range from 0.0 (no habitat value) to 1.0 (ideal habitat). Values should be limited to increments of 0.1 (i.e. do not assign 0.675 because we will enter 0.7 )

\section{Canada Goose}

Cover-Type: Nesting: Riparian Forest (mature cottonwood with open branch structure)

Foraging: Bare (sand gravel), Mud (PAB), Fallow Pasture, Shrub-Steppe/Grasslands, Riparian Herb, Lacustrine, Open Water (POW), Emergent (PEM).

Description: The Canada goose model is for the Yakima Valley region where the geese nest in mature trees within the riparian corridor. Trees or snag must be of sufficient size to support a nest structure and have and open branch structure. When the goslings leave the nest they are lead to an area of with open water, emergent vegetation for cover, and easily accessible herb/grass foraging areas. Both nesting and brood rearing areas have minimal disturbance by human or domestic pets.

HSI Values:

1.0 Riparian forest with some trees or snags $>8$ inches $\mathrm{dbh}$ for nesting. Short grass foraging areas for broods $<1$ mile of nesting areas. Human disturbance $>1 / 2$ mile from foraging and nesting areas.

0.5 Mature trees for nesting limited, few snags. Short grass brood foraging areas either limited or 1 to 2 miles from nesting area. Human disturbance $1 / 4$ to $1 / 2$ miles away.

$0.2 \quad$ Few mature trees for nesting. Brood foraging areas not apparent or $>2$ miles away. Human disturbance $<1 / 4$ miles away.

$0.0 \quad$ No suitable habitat. 


\section{Mallard}

Cover-Type: Nesting: Riparian herb, Grassland, Shrub-grass, Shrub, and Shrub Steppe.

Rearing: Emergent (PEM), Lacustrine, Open Water (POW), Water Lily (PAB).

Description: Nesting habitat is shrub-steppe/grassland and riparian herb cover in proximity to riparian herb habitats for subsequent brood rearing. Broods are moved to areas of emergent vegetation with open water.

HSI Values:

1.0 Nesting: Canopy cover within 300 feet of open water $>75 \%$ and from 300 to 600 feet, cover $>75 \%$. Nesting vegetation is herbaceous or shrub cover from 18 to 24 inches high. Cover provides horizontal and vertical screening. Brood: Canopy cover from 40 to $60 \%$ emergent vegetation (PEM) and/or root floating (water lily $\mathrm{PAB}$ )) with open water being the remainder. Carp absent. Intermittently exposed to semi permanently flooded.

0.5 Nesting: Canopy cover within 300 feet of the water $40 \%$ and from 300 to 600 feet $40 \%$. Nesting cover 12-15 inches high or $25-36$ inches high. Brood cover $20 \%$ open water or $80 \%$ open water. Permanently flooded. Flowing water maintains moderate water clarity.

0.2 Nesting cover within 300 feet of water $20 \%$ and from 300 to 600 feet, $20 \%$. Vegetation $<12$ inches high or $>36$ inches high.

Brood: Open water or solid rooted lily or emergent vegetation. Still or sluggish water, water opaque.

0.0 No suitable habitat.

\section{California Quail}

Cover-Type: Riparian Shrub, Riparian Herb, Fallow Pasture, Pasture, Shrub-Steppe/Grasslands

Description: Forages on seeds and insects in herb and grassy areas with escape cover near by.

HSI Values:

1.0 Herbs and grasses comprise 55 to $70 \%$ of ground cover. Escape cover is shrubs $>7$ feet high. Distance to escape cover $<125$ feet. Patches of escape cover 10 to 20 feet in diameter and distance between patches from 100 to 200 feet.

0.5 Herbs and grass cover $25 \%$. Shrubs $>4$ foot tall. Distance to escape cover (shrubs) $>250$ feet. Distance between escape cover patches either $<50$ feet or $>300$ feet.

0.2 Herb and grass cover $<10 \%$. Shrubs $<3$ feet in height. Distance to escape cover patches $>500$ feet.

0.0 No suitable habitat. 


\section{Great Blue Heron}

Cover Type: Riparian Forest, Bare (Sand/Gravel, Mud (PAB), Grassland, Riverine, Lacustrine, Open Water (POW), Emergent (PEM) ??

Description: Forages along steams, emergent wetland, grasslands and agricultural fields. Tolerates moderate disturbance. In the Yakama Project Area, nests are in riparian forest adjacent to streams or wetlands. Disturbance at either nest sites or foraging areas reduces use.

HSI Values:

1.0 Optimal foraging is in clear water streams, ponds, sloughs, and emergent wetlands, agricultural fields. Nesting trees $<1 / 2$ mile from foraging areas. No substantial human or domestic pet disturbance within $1 / 4$ mile.

0.5 Water not clear in foraging areas. Palustrine trees and or shrubs crowd the stream/pond bank. Moderate disturbance within 1/4 mile. Nesting trees either marginal in size $\left(<8^{\prime \prime} \mathrm{dbh}\right)$ or $>5$ mile from foraging areas.

0.2 Murky water. Appropriate foraging habitat scarce. Foraging area $>6$ miles from nesting area. Frequent human disturbance.

0.0 Either no foraging habitat, no nesting habitat, or persistent high levels of disturbance.

\section{5: Spotted Sandpiper}

Cover-Type: Sand Gravel Cobble Mud (Bare, PAB (mud)

Description: Nest in sparsely vegetated sand/gravel/cobble in braided stream channels. Only limited woody debris present. Nests close to the water. Forages along the waters edge and on mud banks.

HSI Values:

1.0 Herbs $<2$ feet high provide 10 to $50 \%$ cover. Water $<75$ feet from nest sites. Organic debris (driftwood) $<50 \%$. ground cover.

0.5 Herb cover 5 to $10 \%$ or 50 to $75 \%$. From nest sites to water $>200$ feet. Organic debris $>70 \%$ cover.

0.2 Little herb cover or cover $>70 \%$ cover. Distance from nest site to water $>250$ feet. Ground cover mostly organic debris.

0.0 No suitable habitat.

\section{Downy Woodpecker}

Cover-Type:
Description:

HSI Values:

1.0 Forages and nests in mature riparian forest (trees $>12$ " $d b h$ ). Density of

Riparian Forest

Downy woodpeckers glean insects from the bark of trees and excavating into the bark or decaying wood to extract insects. Nests and forages in mature riparian forest. They excavate their nest cavities in dying or decaying trees. snags $>5$ per acre $>6$ inches $\mathrm{dbh}$. Basal area of trees from 40 to 90 sq. $\mathrm{ft}$ per acre and snags density $>5$ per acre. Riparian forest in the project area are assumed to meet these requirements. A territory must have $>10$ acres of suitable habitat.

0.5 Basal area of trees $>30$ sq. $\mathrm{ft}$. per acre and density of snags $>2$ per acre.

0.2 Basal area of trees $>20$ sq. ft. per acre and density of snags $>1$ per acre.

0.0 No nesting or foraging habitat present. 


\section{Black-Capped Chickadee}

\section{Cover-Type: Riparian Forest}

Description: Nests in cavities in trees in mature riparian forest with at least 2 snags per acre. Forages in riparian forest and shrub. Gleans food from foliage, branches, twigs, and bark.

HSI Values:

1.0 Mature riparian forest with trees 50 to $75 \%$ cover and tree height $>50$ feet. Density trees or snags with cavities ( 4 to 8 inches $\mathrm{dbh}$ ) $>2$ per acre.

0.5 Tree canopy $>50 \%$ and trees $>20$ feet in high. At least 1 snag per acre.

0.2 Tree canopy $>10 \%$ cover and trees $>10$ feet in high. Snags density $>0.2$ per acre.

$0.0 \quad$ No habitat present.

\section{Yellow Warbler}

Cover-Type: Riparian Shrub

Description: Forages and nests in tall riparian (hydrophytic) shrubs. All of the riparian shrubs in the study areas are considered hydrophytic. Gleans and hawks for insects.

HSI Values:

1.0 Hydrophytic shrub from 60 to $80 \%$ cover and $>6$ feet high.

0.5 Hydrophytic shrub $<30 \%$ cover and herbs $>3$ feet high.

0.2 Hydrophytic shrubs $<10 \%$ cover and herbs $<18$ inches high.

0.0 No nesting or foraging habitat present.

\section{Meadowlark}

Cover Types: Shrub-Steppe/Grassland/Fallow Pasture/Pasture

Description: Nests and forages in grasslands and open shrub-steppe. Perch sites on shrubs or fence posts in proximity to nest and foraging areas.

HSI Values:

1.0 Grass/herb canopy cover $65 \%$, of which grasses are $65 \%$. Average height of herbs is from 6-13 inches. Shrub cover $<10 \%$. Distance to perch sites $<100$ feet

0.5 Herb canopy $<50 \%$, of which $50 \%$ is grass. Average height of herb canopy less than 5 inches or more than 20 inches. Suitable perch sites $>150$ feet away. Shrub canopy $>20 \%$.

0.2 Herb cover $<25 \%$, of which grass is $<25 \%$. Distance to perch sites is $>200$ feet. Shrub canopy $>30 \%$.

0.0 No suitable habitat. 


\section{Mink}

\section{B-5}

Cover-Type: Riverine, Emergent Wetland, Riparian Forest, Riparian Shrub, Bare (sand and gravel), PAB (mud)

Description: Mink are very adaptable and can effectively use shoreline habitats that provides adequate shelter and structural complexity as long as adequate food supplies are available. Mink use crustaceans (crawfish), fish, amphibians, waterfowl, and small mammals and usually forage on the most abundant food source. In the project area, waterfowl are a major source of food. Human disturbance and/or human simplification of the

HSI Values: riparian shoreline can degrade riparian habitat.

1.0 Tree and/or shrub cover along riparian corridor $>75 \%$. Emergent vegetation 50 to 75 percent. Surface water present $>75 \%$ of year.

0.5 Tree and/or shrub cover along riparian corridor $>50 \%$. Emergent cover $>50 \%$. Surface water present $>50 \%$ of the year.

0.2 Tree and/or shrub cover along riparian corridor $<20 \%$. Surface water present $<30 \%$ of the year.

0.0 No tree or shrub cover in riparian corridor. Surface water only occasionally present. 
Table B-2. Delphi Species HSI Sample Datasheet.

Plot:

Date: End Time:

Start Time:

\section{Serial:}

Elapse (hrs):

Observers:

Area: Satus (S), North Satus (NS), Lower Satus (LS), Wanity Slough (WS), Toppenish Pump (TP), Wapato (W), South Lateral A (SLA), Mosebar (M), Other

Location in Area:

Cover Type: Forest Shrub Herb Herb/Forest Herb/Shrub Shrub/Steppe Ag-fallow Ag-pasture Lacustrine Riverine OpenWater(POW) AquaticBed(PAB) B(SandGraveiCobble) PUB(mud)

Emergent(PEM) Palustrine Shrub(PSS) Riverine/Shrub U(developed) Other:

Add " $\mathrm{e}$ " if exotic comprise more that $50 \%$ of the cover.

Hydrology of Stream or Wetland: absent or highly degraded, function restored, natural or near natural Sample Area: 1 acre circular plot $=118$ foot radius

$1 / 4$ acre circular plot $=59$ foot radius

Plot Number $=$ Initial of Priority Area and sequential plot number. i.e. NS-7

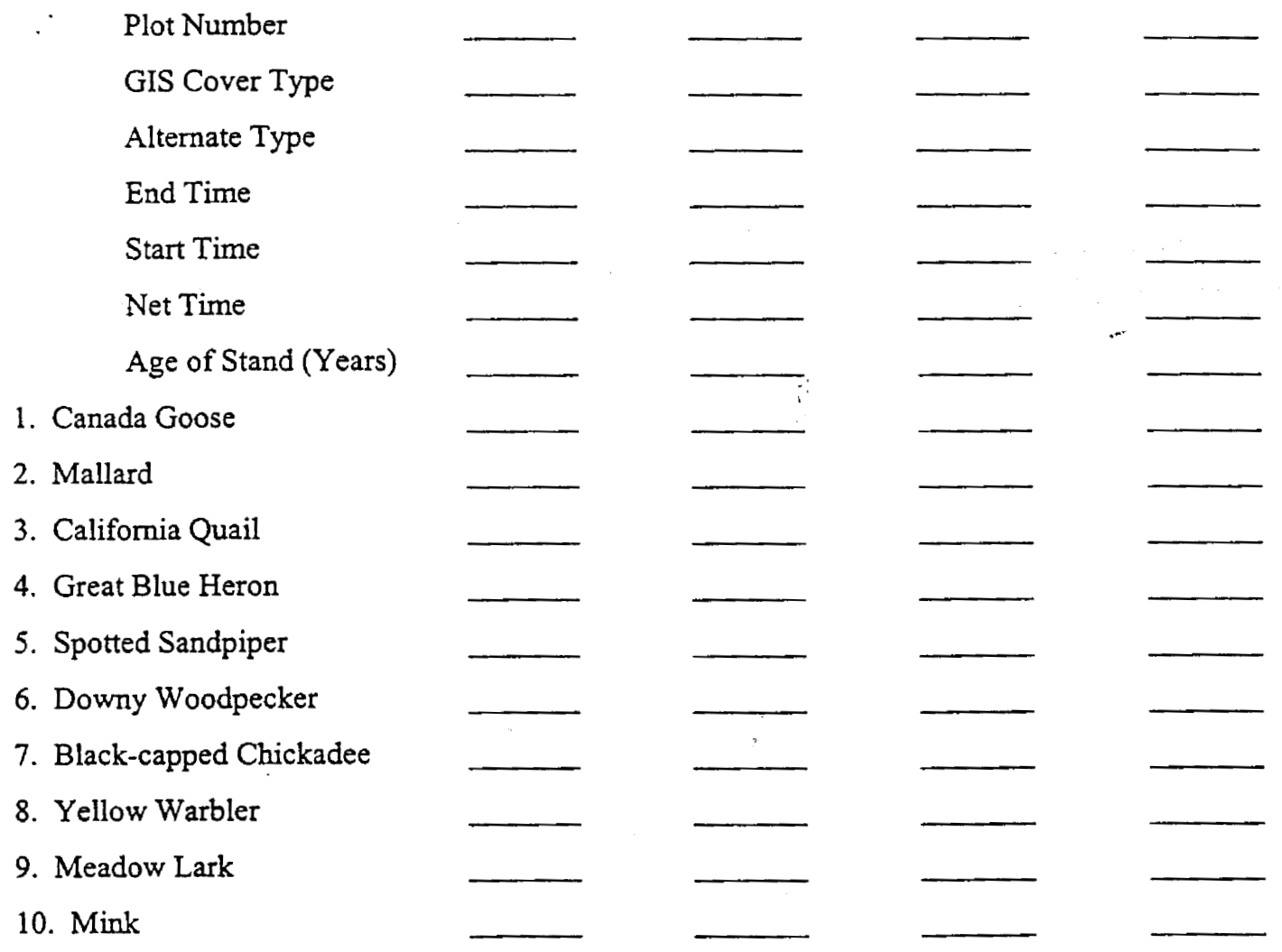




\section{APPENDIX C}

Yakama Nation Method 


\begin{tabular}{|c|c|c|c|c|c|c|c|c|c|c|c|c|c|}
\hline $\mathrm{X}$ & & & & & & & & & & $2.100 \perp \partial d$ & Hanuos & 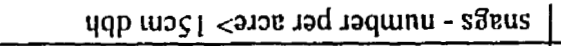 & $\angle \varepsilon$ \\
\hline \multirow[t]{37}{*}{$\mathrm{x}$} & & & & & & & & & & op/u! bs & & ajov dad raje [eseq - soan] & $9 \varepsilon$ \\
\hline & $\mathrm{x}$ & & & & & & & & & & & 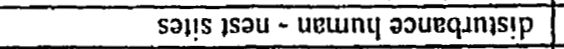 & $\widehat{\overparen{S E}}$ \\
\hline & $x$ & & & & & & & & & & & K!!!!qe|!ese - sa!!s isau & $\nabla \varepsilon$ \\
\hline & $x$ & & & & & & & & & & & sease 8u!gejoj - uruny oourqun!s!p & 'EE \\
\hline & $X$ & & & & & & & & & & & 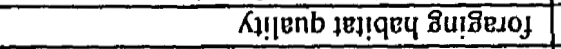 & $\tau \varepsilon$ \\
\hline & $\mathrm{X}$ & & & & & & & & & 1ә2 & $u$ & 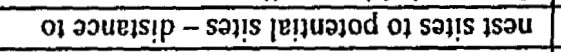 & $\cdot I \varepsilon$ \\
\hline & $\mathrm{X}$ & & & & & & & & & 1021 & $\boldsymbol{w}$ & seว.1 gu!seıoj of วoukis!p - sa!!s łsau & .OE \\
\hline & & $\mathrm{X}$ & & & & & & & & เәА०0 \% & & 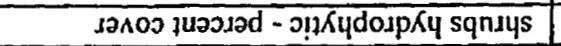 & .62 \\
\hline & & $X$ & & & & & & & & มอก0ว \% & & 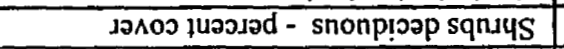 & 82 \\
\hline & & & $\mathrm{X}$ & & & & & & & & 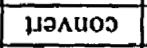 & 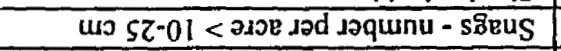 & $\angle 2$ \\
\hline & & & $x$ & & & & & & & 1001 & $\mathbf{u}$ & 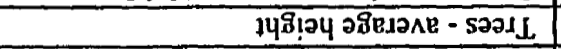 & 92 \\
\hline & & & & $\mathrm{X}$ & & & & & & 10गj & $\mathrm{u}$ & 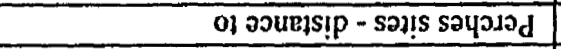 & s2 \\
\hline & & & & $\mathrm{X}$ & & & & & & soyou! & wo & 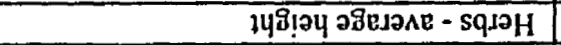 & $\pi \tau$ \\
\hline & & & & $\mathrm{X}$ & & & & & & $.12 \wedge 00 \%$ & & 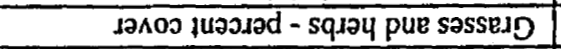 & $\varepsilon z$ \\
\hline & & & & $\mathrm{X}$ & & & & & & $10 \wedge 00 \%$ & & 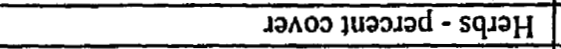 & $2 z$ \\
\hline & & & & & $\mathrm{X}$ & & & & & $10 \wedge 00 \%$ & & 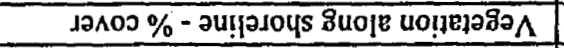 & .12 \\
\hline & & & & & $\mathrm{X}$ & & & & & $12 \wedge 03 \%$ & & 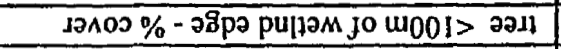 & .02 \\
\hline & & & & & $\mathrm{X}$ & & & & & JOAO0 \% & & 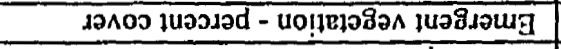 & 61 \\
\hline & & & & $\mathrm{X}$ & $x$ & & & & & $19 \Lambda 00 \%$ & & 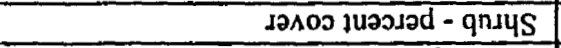 & 81 \\
\hline & & & $X$ & & $\mathrm{X}$ & & & & & دว^00 \% & & 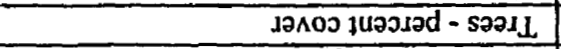 & $\angle I$ \\
\hline & & & & & $X$ & & & & & $\%$ & & 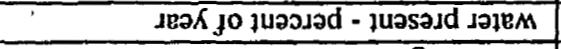 & 91 \\
\hline & & & & & & $\mathrm{X}$ & & & & $12 \Lambda 00 \%$ & & 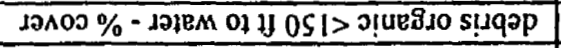 & 'SI \\
\hline & & & & & & $\mathrm{X}$ & & & & 1021 & யง & 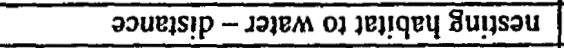 & $\because 1$ \\
\hline & & & & & & $x$ & & & & $12 \wedge 00 \%$ & & 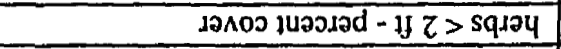 & $\varepsilon I$ \\
\hline & & & & & & & $\mathrm{X}$ & & & & & 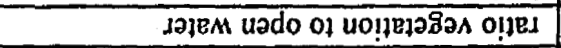 & ?2I \\
\hline & & & & & & & $\mathrm{X}$ & & & $12 \wedge 00 \%$ & & 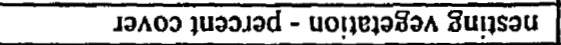 & 111 \\
\hline & & & & & & & $\mathrm{X}$ & & & səu’u! & us & $148124-12 \wedge 00$ [enp!sว1 & $\mathrm{OI}$ \\
\hline & & & & & & & $x$ & & & 102] & $u$ & 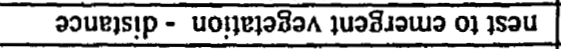 & .6 \\
\hline & & & & & & & $\mathrm{X}$ & & & & & $\dot{\alpha i}$ pJe||eu & \\
\hline & & & & & & & $\mathrm{X}$ & $\mathrm{X}$ & & 122,1 & $u$ & วoueıs!p - ueuny әourqunis!p & 8 \\
\hline & & & & & & & & $x$ & & 1әวิ & $\underline{u}$ & 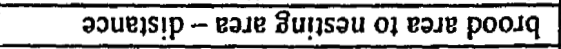 & $\ddot{L}$ \\
\hline & & & & & & & & $\mathrm{X}$ & & & & 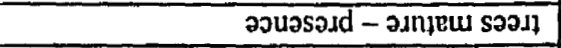 & 9 \\
\hline & & & & & & & & & $X$ & 1201 & $\mathrm{u}$ & 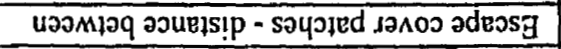 & s \\
\hline & & & & & & & & & $\mathrm{X}$ & 100 & $u$ & 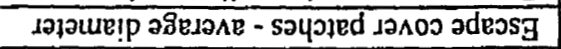 & $\ddot{b}$ \\
\hline & & & & & & & & & $\mathrm{X}$ & 1003 & $\mathrm{u}$ & 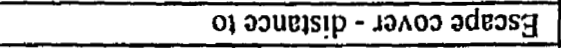 & $\varepsilon$ \\
\hline & & $\mathrm{X}$ & & & & & & & $\mathrm{X}$ & sayou! & wo & 148124 ว8อ & $z$ \\
\hline & & & & & & & & & $\mathrm{X}$ & $10 \wedge 02 \%$ & & $10 \wedge 03$ Kdoues juaวsod - squa 8 ssejo & -1 \\
\hline $\begin{array}{l}\text { Ydpoos } \\
\text { Kuмop }\end{array}$ & $\begin{array}{l}\text { 10.194 } \\
\text { nाq } 208\end{array}$ & $\begin{array}{c}\text { Аग्वसहM } \\
\text { моाןर }\end{array}$ & $\begin{array}{l}\text { әэри } \\
\text { भग!ฺ }\end{array}$ & $\begin{array}{c}\text { Y.xt } \\
\text { มореכu }\end{array}$ & yụı & $\begin{array}{l}\text { dodi!d } \\
\text { purs }\end{array}$ & pdeıleur & $\begin{array}{c}\text { os009 } \\
\text { tpeues }\end{array}$ & $\begin{array}{l}\text { I!\&nb } \\
J ![60\end{array}$ & $\begin{array}{c}\mathrm{d} \\
\text { HองuoJ }\end{array}$ & $\begin{array}{c}\text { I!un } \\
\text { ppow }\end{array}$ & มวןวนหนย & \\
\hline
\end{tabular}

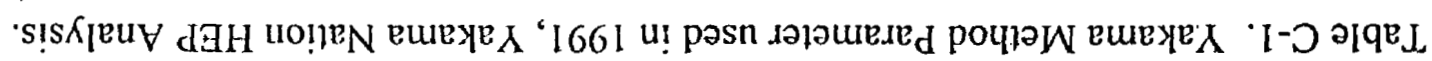


Table C-2. Yakama Method Parameters Sampling Order, Yakama Nation HEP Analysis.

\begin{tabular}{|c|c|c|}
\hline & Model Parameter & Proposed Unit \\
\hline & TREES - riparian forest only & \\
\hline 1 & Snags/ac. 4 to $8 " \mathrm{dbh}$ & \#/ac. \\
\hline 2 & Snags/ac. $>6$ " dbh & $\# . \mathrm{ac}$ \\
\hline 3 & Basal area/ac. & sq. in./ac. \\
\hline 4 & Tree canopy cover & percent \\
\hline 5 & Average tree height & feet \\
\hline \multirow[t]{2}{*}{6} & Mature trees present & yes/no \\
\hline & $\begin{array}{l}\text { SHRUBS - riparian forest, riparian shrub, shrub- } \\
\text { steppe }\end{array}$ & \\
\hline 7 & Shrub canopy cover & percent \\
\hline 8 & Deciduous shrub cover & percent \\
\hline 9. & Hydrophytic shrub cover & percent \\
\hline \multirow[t]{2}{*}{10} & Average shrub height & feet (decimal) \\
\hline & $\begin{array}{l}\text { HERBS and GRASS - riparian forest, riparian shrub, } \\
\text { riparian herb, shrub-steppe, agriculture }\end{array}$ & \\
\hline 11 & Herb cover & percent \\
\hline 12 & Grass cover & percent \\
\hline 13 & Ratio of grass to herb & ratio ... \\
\hline 14 & Cover herbs $<2$ feet height & percent \\
\hline \multirow[t]{2}{*}{15} & Average herb height & feet \\
\hline & EMERGENT PLANTS - PEM & \\
\hline \multirow[t]{2}{*}{16} & Cover of emergent plants & percent \\
\hline & SAND, GRAVEL, COBBEL, MUD - (B) & \\
\hline \multirow[t]{3}{*}{17} & Cover by organic debris and driftwood & percent \\
\hline & SPECIES SPECIFIC PARAMETERS & \\
\hline & $\begin{array}{l}\text { California Quail - riparian shrub, riparian herb, } \\
\text { shrub-steppe }\end{array}$ & \\
\hline 18 & distance to escape cover - (dense vegetation $>8$ " high) ??? & feet \\
\hline 19 & average diameter of patches escape cover & feet \\
\hline \multirow[t]{2}{*}{20} & distance between escape cover patches & feet \\
\hline & $\begin{array}{l}\text { Canada Goose - riparian herb, shrub-steppe, } \\
\text { agriculture, sand/gravel }\end{array}$ & \\
\hline 21 & quality of nesting habitat & score \\
\hline 22 & distance from nesting to brood areas & feet \\
\hline 23 & distance from nest to human disturbance & feet \\
\hline
\end{tabular}


Table C-2. Continued.

Mallard - riparian herb, emergent, shrub-steppe, agriculture, riverine, lacustrine

$24 ? ?$

$25 ? ?$

26 distance from nest site to emergent plants

27 height of residual cover

28 cover of nesting habitat

29 level of human disturbance

30 ratio of emergent vegetation to open water Spotted Sandpiper - sand/gravel

31 distance of nest site to water Mink - riparian forest, riparian shrub, emergent, riverine, sand/gravel

32 open water during the year

percent

33 . trees and shrubs cover <330" wetland edge. percent

34. tree and shrub cover along shoreline

percent

Western Meadowlark - shrub-steppe

35 distance to perch sites

feet

Great Blue Heron - riparian forest, shrub-steppe, riverine, lacustrine, sand/gravel

36 distance foraging area to potential nest sites miles

37 foraging habitat quality score

38 level of human disturbance near potential feeding areas score

39 availability of potential nest sites score

40 level human disturbance potential nest sites score

41 distance between active and potential nest sites miles 
Table C-3. YN Field Data Sheet - Estimate YN-HSI Values. Shrub-Steppe, Grassland, Herb, Agriculture

Plot:

Serial:

Date: Start Time: End Time: Elapse:

Observers:

Area: Satus (S), North. Satus (NS), Lower Satus (LS), Wanity Slough (WS), Toppenish Pump (TP), Wapato (W), South Lateral A (SLA), Mosebar (M)

Location in Area:

Conditions/Treatment:

Exotic Shrub Cover: $0 \%, 1-5 \%, 6-25 \%, 26-50 \%, 51-75 \%,>75 \%$

Exotic Grass/Herb Cover:

$0 \%, 1-5 \%, 6-25 \%, 26-50 \%, 51-75 \%,>75 \%$

Cover Type: Forest Shrub Herb Herb/Forest Herb/Shrub Shrub/Steppe Ag-fallow Ag-pasture Lacustrine Riverine OpenWater(POW) AquaticBed(PAB) Water Lily (PAB4) SGCM(SandGravelCobble) Cattail-Bulrush(emergent PEM) Palustrine Shrub(PSS) Other Photo Roll: Exposures:

Hÿdrology of Stream or Wetland: absent or highly degraded, function restored, natural or near natural Stand Age: (explain basis of estimate)

Sample Area: 1 acre circular plot $=118$ foot radius

$1 / 4$ acre circular plot $=59$ foot radius

\begin{tabular}{|l|c|c|c|c|c|c|c|}
\hline SAND GRAVEL MUD & & & & & & & \\
\hline percent of area & none & solitary & $1-5 \%$ & $6-25 \%$ & $26-50 \%$ & $51-75 \%$ & $76-100 \%$ \\
\hline percent cover organic debris & none & solitary & $1-5 \%$ & $6-25 \%$ & $26-50 \%$ & $51-75 \%$ & $76-100 \%$ \\
\hline SHRUBS & & & & & & & \\
\hline percent cover & & & & & & & \\
\hline percent cover deciduous & none & Solitary & $1-5 \%$ & $6-25 \%$ & $26-50 \%$ & $51-75 \%$ & $76-100 \%$ \\
\hline percent cover hydrophytic & none & Solitary & $1-5 \%$ & $6-25 \%$ & $26-50 \%$ & $51-75 \%$ & $76-100 \%$ \\
\hline Average height & 0 & $<1 \mathrm{ft}$ & $1-5 \%$ & $6-25 \%$ & $26-50 \%$ & $51-75 \%$ & $76-100 \%$ \\
\hline & & & & $3-6 \mathrm{ft}$ & $6-10 \mathrm{ft}$ & $>10 \mathrm{ft}$ & \\
\hline HERBS \& GRASS & & & & & & & \\
\hline percent herb \& grass & none & solitary & $1-5 \%$ & $6-25 \%$ & $26-50 \%$ & $51-75 \%$ & $76-100 \%$ \\
\hline grass as percent of g+h & None & Solitary & $1-5 \%$ & $6-25 \%$ & $26-50 \%$ & $51-75 \%$ & $76-100 \%$ \\
\hline percent cover <2 ft & None & Solitary & $1-5 \%$ & $6-25 \%$ & $26-50 \%$ & $51-75 \%$ & $76-100 \%$ \\
\hline Average height & 0 & $<1 \mathrm{ft}$ & $1-3 \mathrm{ft}$ & $3-6 \mathrm{ft}$ & $6-10 \mathrm{ft}$ & $>10 \mathrm{ft}$ & \\
\hline & & & & & & & \\
\hline TREES & 1 & 2 & 3 & 4 & 5 & 6 & 7 \\
\hline mature trees present & No & Yes & & & & & \\
\hline & & & & & & & \\
\hline HUMAN DISTURBANCE & none & $<500$ & $<1 / 4$ mile & $1 / 41 / 2 \mathrm{mile}$ & $>1 / 2 \mathrm{mile}$ & $>1 \mathrm{mile}$ & $>5 \mathrm{miles}$ \\
\hline
\end{tabular}


Table C-3. Continued.

Plot:

Shrub, Shrub-Steppe, Grassland, Herb, Agriculture

Serial:

\begin{tabular}{|c|c|c|c|c|c|c|c|}
\hline $\begin{array}{l}\text { California Quail } \\
\text { S, Ag, SS/G, G, H }\end{array}$ & & & & & & & \\
\hline $\begin{array}{l}\text { Distance to escape cover } \\
\text { escape cover }=>8 \mathrm{ft} \text { high }\end{array}$ & 0 & $<100 \mathrm{ft}$ & $101-180 \mathrm{ft}$ & $\begin{array}{l}181- \\
300 \mathrm{ft}\end{array}$ & $\begin{array}{l}301- \\
500 \mathrm{ft}\end{array}$ & $\begin{array}{l}501- \\
874 \mathrm{ft}\end{array}$ & $>875 \mathrm{ft}$ \\
\hline av diameter escape patches & None & $0-10 \mathrm{ft}$ & $11.20 \mathrm{ft}$ & $>20 \mathrm{ft}$ & & & \\
\hline Distance to escape patches & None & $<30 \mathrm{ft}$ & $31-90 \mathrm{ft}$ & $91-200 \mathrm{ft}$ & $\begin{array}{l}201- \\
300 \mathrm{ft}\end{array}$ & $>300 \mathrm{ft}$ & \\
\hline $\begin{array}{l}\text { Canada Goose } \\
\text { nests in riparian trees } \\
\text { Agp, Agf, SSG, H }\end{array}$ & & & & & & & \\
\hline Quality of foraging habitat & & & & & & & \\
\hline $\begin{array}{l}\text { Mallard } \\
\text { Nest: H, G, S, SSG, Ag-pf }\end{array}$ & & & & & & & \\
\hline Distance to emergent plants & 0 & $<1 / 4$ mile & $\begin{array}{l}1 / 4 \text { to } 3 / 4 \\
\text { mile }\end{array}$ & $\begin{array}{l}>3 / 4 \\
\text { mile }\end{array}$ & & & \\
\hline $\begin{array}{l}\text { height of nesting cover } \\
\text { providing both horizontal } \\
\text { and vertical screen of nest }\end{array}$ & None & $1-15$ in & $16-24$ in & $25-48$ in & $>48$ in & & \\
\hline Percent cover nest habitat & None & solitary & $1.5 \%$ & $6-25 \%$ & $26-50 \%$ & $51-75 \%$ & $76-100 \%$ \\
\hline $\begin{array}{l}\text { ratio of lily pads, cattail, or } \\
\text { bulrush to open water }\end{array}$ & None & $<40: 60$ & $\begin{array}{l}40: 60- \\
60: 40 \\
\end{array}$ & $>60-40$ & & & \\
\hline $\begin{array}{l}\text { Western Meadowlark } \\
\text { SS/G, Agp, Agf }\end{array}$ & & & & & & & \\
\hline Distance to perch sites & $\begin{array}{c}\text { No } \\
\text { perches }\end{array}$ & Few & scattered & abundant & & & \\
\hline $\begin{array}{l}\text { Great Blue Heron } \\
\text { Foraging: } S, G, R, L, P O W, \\
\text { PEM, PSS, } A g-p f\end{array}$ & 1 & 2 & 3 & 4 & 5 & 6 & 7 \\
\hline Distance foraging to nest & 0 & $<500^{\prime}$ & $<1 / 4$ mile & $1 / 41 / 2$ mile & $>1 / 2$ mile & $>1$ mile & $>5$ miles \\
\hline Quality of foraging habitat & none & Dry ag-pf & Wet ag-pf & Shlw mrk & shlw clr & Dp mrk & $\mathrm{Dp}$ clr \\
\hline potential nest sites & no & yes & & & & & \\
\hline $\begin{array}{l}\text { Mink } \\
\text { F, S, R, PEM, PSS }\end{array}$ & & & & & & & \\
\hline percent of year with water & 0 & $1-25 \%$ & $26-50 \%$ & $51-75 \%$ & $100 \%$ & & \\
\hline $\begin{array}{l}\text { percent tree/shrub cover } \\
\text { within } 330 \text { feet water edge }\end{array}$ & none & Solitary & $1-5 \%$ & $6-25 \%$ & $26-50 \%$ & $51-75 \%$ & $76-100 \%$ \\
\hline $\begin{array}{l}\text { percent tree/shrub cover } \\
\text { along shoreline }\end{array}$ & none & Solitary & $1-5 \%$ & $6-25 \%$ & $26-50 \%$ & $51-75 \%$ & $76-100 \%$ \\
\hline
\end{tabular}


Table C-4. YN Datasheet - Estimate YN-HSI Values.

Plot:

\section{Trees and Shrubs}

Serial:

Date: Start Time: End Time: Elapse:

Observers:

Area: Satus (S), North. Satus (NS), Lower Satus (LS), Wanity Slough (WS), Toppenish Pump (TP), Wapato (W), South Lateral A (SLA), Mosebar (M)

Location in Area:

Conditions/Treatment:

Exotic Shrub Cover: $0 \%, 1-5 \%, 6-25 \%, 26-50 \%, 51-75 \%,>75 \%$

Exotic Grass/Herb Cover: $0 \%, 1-5 \%, 6-25 \%, 26-50 \%, 51-75 \%,>75 \%$

GIS Cover Type: Forest Shrub Herb Herb/Forest Herb/Shrub Shrub/Steppe Ag-fallow Ag-pasture SGCM(SandGravelCobble) Lacustrine Riverine OpenWater(POW) AquaticBed(PAB) Water Lily (PAB4) PUB(mud) Cattail-Bulrush(emergent PEM) Palustrine Shrub(PSS) Other Aerial Photo:

\section{GIS/GPS Coordinates:}

Photo Roll: Exposures: Reoccupied or New Site

Hydrology of Stream or Wetland: absent or highly degraded, function restored, natural or near natural Land Use: hunt \& non-consumptive rec, hunt \& YN gathering, close - YN gathering, closed Stand Age: (explain basis of estimate)

Sample Area: 1 acre circular plot $=118$ foot radius $1 / 4$ acre circular plot $=59$ foot radius

\begin{tabular}{|l|c|c|c|c|c|c|c|}
\hline \multicolumn{1}{|c|}{ TREES } & 1 & 2 & 3 & 4 & 5 & 6 & 7 \\
\hline mature trees present & no & yes & i & & & & \\
\hline percent canopy & none & solitary & $1-5 \%$ & $6-25 \%$ & $26-50 \%$ & $51-75 \%$ & $76-100 \%$ \\
\hline average height & 0 & $<10 \mathrm{ft}$ & $10-25 \mathrm{ft}$ & $26-50 \mathrm{ft}$ & $51-100 \mathrm{ft}$ & $>100 \mathrm{ft}$ & \\
\hline average dbh & 0 & $<4$ in & $4-8 \mathrm{in}$ & $8-12 \mathrm{in}$ & $12-24 \mathrm{in}$ & $24-36$ & $>36 \mathrm{in}$ \\
\hline maximum dbh & 0 & $<4$ in & $4-8 \mathrm{in}$ & $8-12 \mathrm{in}$ & $12-24 \mathrm{in}$ & $24-36$ & $>36 \mathrm{in}$ \\
\hline snags 4-6 in per ac & 0 & $1-5$ & $6-10$ & $11-20$ & $>20$ & & \\
\hline snags >6 in per ac & 0 & $1-5$ & $6-10$ & $11-20$ & $>20$ & & \\
\hline & & & & & & & \\
\hline SHRUBS & & & & & & & \\
\hline percent cover & none & solitary & $1-5 \%$ & $6-25 \%$ & $26-50 \%$ & $51-75 \%$ & $76-100 \%$ \\
\hline percent cover deciduous & none & solitary & $1-5 \%$ & $6-25 \%$ & $26-50 \%$ & $51-75 \%$ & $76-100 \%$ \\
\hline percent cover hydrophytic & none & solitary & $1-5 \%$ & $6-25 \%$ & $26-50 \%$ & $51-75 \%$ & $76-100 \%$ \\
\hline average height & 0 & $<1 \mathrm{ft}$ & $1-3 \mathrm{ft}$ & $3-6 \mathrm{ft}$ & $6-10 \mathrm{ft}$ & $>10 \mathrm{ft}$ & \\
\hline & & & & & & & \\
\hline \multicolumn{1}{|c|}{ HERBS \& GRASS } & & & & & & & \\
\hline percent cover & none & solitary & $1-5 \%$ & $6-25 \%$ & $26-50 \%$ & $51-75 \%$ & $76-100 \%$ \\
\hline percent herb \& grass & none & solitary & $1-5 \%$ & $6-25 \%$ & $26-50 \%$ & $51-75 \%$ & $76-100 \%$ \\
\hline grass as percent of g+h & None & 0 & $1-5 \%$ & $6-25 \%$ & $26-50 \%$ & $51-75 \%$ & $76-100 \%$ \\
\hline percent cover <2 ft & None & solitary & $1-5 \%$ & $6-25 \%$ & $26-50 \%$ & $51-75 \%$ & $76-100 \%$ \\
\hline average height & 0 & $<1 \mathrm{ft}$ & $1-3 \mathrm{ft}$ & $3-6 \mathrm{ft}$ & $6-10 \mathrm{ft}$ & $>10 \mathrm{ft}$ & \\
\hline
\end{tabular}


Table C-4. Continued.

C-7

Trees and Shrubs

Plot:

Serial:

\begin{tabular}{|c|c|c|c|c|c|c|c|}
\hline $\begin{array}{l}\text { Mink } \\
\text { F, S, R, PEM, PSS }\end{array}$ & & & & & & & \\
\hline percent of year with water & 0 & $1-25 \%$ & $26-50 \%$ & $51-75 \%$ & $100 \%$ & & \\
\hline $\begin{array}{l}\text { percent tree/shrub cover } \\
\text { within } 330 \text { feet water edge }\end{array}$ & none & solitary & $1-5 \%$ & $6-25 \%$ & $26-50 \%$ & $51-75 \%$ & $\begin{array}{c}76-100 \\
\%\end{array}$ \\
\hline $\begin{array}{l}\text { percent tree/shrub cover } \\
\text { along shoreline }\end{array}$ & none & solitary & $1-5 \%$ & $6-25 \%$ & $26-50 \%$ & $51-75 \%$ & $\begin{array}{c}76-100 \\
\%\end{array}$ \\
\hline $\begin{array}{l}\text { Great Blue Heron } \\
\text { F, S, G, R, L, POW, B, } \\
\text { PAB, PEM, PSS, Agf, Agp }\end{array}$ & & & & & & & \\
\hline $\begin{array}{l}\text { distance from foraging areas } \\
\text { to nest }\end{array}$ & $x \mathrm{xx}$ & & & & & & \\
\hline quality of foraging habitat & none & $\begin{array}{c}\text { wet } \\
\text { pasture }\end{array}$ & $\begin{array}{l}\text { shallow } \\
\text { clear } \mathrm{H}_{2} \mathrm{O}\end{array}$ & & & & \\
\hline $\begin{array}{l}\text { level of human disturbance } \\
\text { potential foraging areas }\end{array}$ & & $\begin{array}{l}\text { frequent } \\
<330 \mathrm{ft}\end{array}$ & $\begin{array}{c}\text { not } \\
\text { frequent }\end{array}$ & & & & \\
\hline $\begin{array}{l}\text { availability of nest sites trees } \\
>18 . \mathrm{ft} \text { high, open canopy, } \\
<750 \mathrm{ft} \text { to water }\end{array}$ & none & present & & & & & \\
\hline $\begin{array}{l}\text { level of human disturbance } \\
\text { potential nest sites }\end{array}$ & $\begin{array}{l}\text { land } \\
\text { water }\end{array}$ & $\begin{array}{l}<1 / 4 \text { mile } \\
<500 \mathrm{ft}\end{array}$ & $\begin{array}{l}>1 / 4 \text { mile } \\
>500 \mathrm{ft}\end{array}$ & & & & \\
\hline $\begin{array}{l}\text { distance between active and } \\
\text { potential nest sites }\end{array}$ & $\mathrm{xxx}$ & $\mathrm{xxx}$ & & & & & \\
\hline & & & & & & & \\
\hline
\end{tabular}


Table C-5. YN Datasheet - Estimate YN-HSI Values.

C-8

Lacustrine - Riverine - POW - PAB - PAB4 - PEM

Plot:

Sand/Gravel/Mud

page 1

Date: Start Time:

End Time:

Elapse:

Observers:

Area: Satus (S), North. Satus (NS), Lower Satus (LS), Wanity Slough (WS), Toppenish Pump (TP),

Wapato (W), South Lateral A (SLA), Mosebar (M)

Location in Area:

Conditions/Treatment:

Exotic Cover: $0 \%, 1-5 \%, 6-25 \%, 26-50 \%, 51-75 \%,>75 \%$

GIS Cover Type: Forest Shrub Herb Herb/Forest Herb/Shrub Shrub/Steppe Ag-fallow

Ag-pasture Lacustrine Riverine OpenWater(POW) AquaticBed(PAB) Water Lily (PAB4)

B(SandGravelCobble) PUB(mud) Cattail-Bulrush(emergent PEM) Palustrine Shrub(PSS) Other

Aerial Photo:

GIS/GPS Coordinates:

Photo Roll:

Exposures:

Reoccupied or New Site

Hydrology of Stream or Wetland: absent or highly degraded, function restored, natural or near natural

Land Use: hunt \& non-consumptive rec, hunt \& YN gathering, close - YN gathering, closed

Stand Age: (explain basis of estimate)

Sample Area: 1 acre circular plot $=118$ foot radius

$1 / 4$ acre circular plot $=59$ foot radius

\begin{tabular}{|c|c|c|c|c|c|c|c|}
\hline \multicolumn{8}{|l|}{ AQUATIC-RIVERINE } \\
\hline percent cover floating & none & solitary & $1-5 \%$ & $6-25 \%$ & $26-50 \%$ & $51-75 \%$ & $76-100 \%$ \\
\hline percent cover sedge/rush & none & solitary & $1.5 \%$ & $6-25 \%$ & $26-50 \%$ & $51.75 \%$ & $76-100 \%$ \\
\hline & 1 & 2 & 3 & 4 & 5 & 6 & 7 \\
\hline Sand Gravel Mud & & & & & & & \\
\hline percent of area & none & solitary & $1-5 \%$ & $6-25 \%$ & $26-50 \%$ & $51-75 \%$ & $\begin{array}{c}76-100 \\
\%\end{array}$ \\
\hline percent cov organic debris & none & solitary & $1-5 \%$ & $6-25 \%$ & $26-50 \%$ & $51-75 \%$ & $\begin{array}{c}76-100 \\
\%\end{array}$ \\
\hline $\begin{array}{l}\text { Mallard } \\
\text { Nesting: H, G, SG, S, SS } \\
\text { Brood: L, POW, PAB, PEN }\end{array}$ & & & - & & & & \\
\hline $\begin{array}{l}\text { distance from nest to } \\
\text { emergent plants }\end{array}$ & $\begin{array}{l}>3 / 4 \\
\text { mile }\end{array}$ & $\begin{array}{l}1 / 4 \text { to } 3 / 4 \\
\text { mile }\end{array}$ & $<1 / 4$ mile & & & & \\
\hline height of residual cover & $\begin{array}{l}1 / 15 \\
\text { in }\end{array}$ & $16-24$ in & $25-48$ in & $>48$ in & & & \\
\hline percent cover nest habitat & none & solitary & $1-5 \%$ & $6-25 \%$ & $26-50 \%$ & $51-75 \%$ & $\begin{array}{c}76-100 \\
\%\end{array}$ \\
\hline level of human disturbance & none & low & Moderate & high & & & \\
\hline ratio PEM to open water & $\begin{array}{c}<40: 6 \\
0\end{array}$ & $\begin{array}{l}40: 60- \\
60: 40\end{array}$ & $>60-40$ & & & & \\
\hline
\end{tabular}


Table C-5. Continued.

Plot:

Lacustrine - Riverine - POW - PAB - PAB4 - PEM

Serial:

Sand/Gravel/Mud

\begin{tabular}{|c|c|c|c|c|c|c|c|}
\hline \multicolumn{8}{|l|}{$\begin{array}{l}\text { Spotted Sandpiper } \\
\text { sand, gravel, cobble, mud }\end{array}$} \\
\hline distance nest sites to water & & & & & & & \\
\hline \multicolumn{8}{|l|}{$\begin{array}{l}\text { Mink } \\
\text { F, S, R, PEM, PSS }\end{array}$} \\
\hline percent of year with water & 0 & $1.25 \%$ & $26-50 \%$ & $51-75 \%$ & $100 \%$ & & \\
\hline $\begin{array}{l}\text { percent tree/shrub cover } \\
\text { within } 330 \text { feet water edge }\end{array}$ & none & solitary & $1-5 \%$ & $6-25 \%$ & $26-50 \%$ & $51-75 \%$ & $\begin{array}{c}76-100 \\
\% \\
\end{array}$ \\
\hline $\begin{array}{l}\text { percent tree/shrub cover } \\
\text { along shoreline }\end{array}$ & none & solitary & $1-5 \%$ & $6-25 \%$ & $26-50 \%$ & $51-75 \%$ & $\begin{array}{c}76-100 \\
\%\end{array}$ \\
\hline $\begin{array}{l}\text { Great Blue Heron } \\
\text { nesting: } F \\
\text { foraging: } S, G, R, L, P O W, \\
B, P A B, P E M, P S S, A g\end{array}$ & 1 & 2 & 3 & 4 & 5 & 6 & 7 \\
\hline $\begin{array}{l}\text { distance from foraging areas } \\
\text { to nest }\end{array}$ & $\mathrm{xxx}$ & & & & & & \\
\hline quality of foraging habitat & none & $\begin{array}{c}\text { wet } \\
\text { pasture }\end{array}$ & $\begin{array}{l}\text { shallow } \\
\text { clear } \mathrm{H}_{2} \mathrm{O}\end{array}$ & & & & \\
\hline $\begin{array}{l}\text { level of human disturbance } \\
\text { potential foraging areas }\end{array}$ & & $\begin{array}{l}\text { frequent } \\
<330 \mathrm{ft} \\
\end{array}$ & $\begin{array}{c}\text { not } \\
\text { frequent }\end{array}$ & & & & \\
\hline $\begin{array}{l}\text { availability of nest sites trees } \\
>18 \mathrm{ft} \text { high, open canopy, } \\
<750 \mathrm{ft} \text { to water }\end{array}$ & none & present & & & & & \\
\hline $\begin{array}{l}\text { level of human disturbance } \\
\text { potential nest sites }\end{array}$ & $\begin{array}{l}\text { land } \\
\text { water }\end{array}$ & $\begin{array}{l}<1 / 4 \text { mile } \\
<500 \mathrm{ft}\end{array}$ & $\begin{array}{l}>1 / 4 \text { mile } \\
>500 \mathrm{ft}\end{array}$ & & $\cdots$ & & \\
\hline $\begin{array}{l}\text { Distance between active and } \\
\text { potential nest sites }\end{array}$ & $\mathrm{xxx}$ & $\mathrm{xxx}$ & & & & & \\
\hline & & & & & & & \\
\hline
\end{tabular}


Table C-6. Yakama Method Field Estimates, Ordered by Plot Serial Number.

Values are the column checked on field datasheet.

saved as $\mathrm{Tb}-64-68 . \mathrm{xls}$, sheet: $\mathrm{Tb}-66$

\begin{tabular}{|c|c|c|c|c|c|c|c|c|c|c|}
\hline Serial Number & 1 & 2 & 3 & 4 & 5 & 6 & 7 & 8 & 9 & 10 \\
\hline Plot Number & SLA-1 & SLA-2 & SLA-3 & SLA-4 & SLA-S & SLA-6 & SLA-7 & SLA-8 & SLA-9 & SLA-10 \\
\hline Date & $8 / 9 / 99$ & $8 / 9 / 99$ & $8 / 9 / 99$ & $8 / 9 / 99$ & $8 / 9 / 99$ & $8 / 9 / 99$ & $8 / 9 / 99$ & 8/9/999 & $8 / 9 / 99$ & $8 / 9199$ \\
\hline Observers & all & all & all & all & RSF et al & RSF et al & RSF et al & RSF et al & TH,A,H & $\mathrm{TH}, \mathrm{A}, \mathrm{H}$ \\
\hline Wildlife Area & S Lat A & $S$ Lat $A$ & $S$ Lat $A$ & $S$ Lat $\mathrm{A}$ & $S$ Lat $A$ & S Lat A & S Lat A & S Lat A & S Lat A & S Lat A \\
\hline 1991 Cover Type & SSG & PEM & SSG & $\mathrm{Ag}$ & $\mathbf{A g}$ & L & $\mathrm{Ag}$ & PEM & PEM & $\mathrm{rF}$ \\
\hline 1999 Cover Type & SSG & PEM & SSG & $\mathbf{A g}$ & $\mathrm{Ag}$ & $\mathbf{L}$ & $\mathrm{Ag}$ & PEM & PEM & $\mathbf{r} \mathbf{F}$ \\
\hline RAI Cover Type & G & PEM & $\mathbf{G}$ & Ag-pf & Ag-pf & POW & Ag-pf & PEM & PEM & $\mathbf{F}$ \\
\hline$\%$ Exotic Shrubs & 1 & 1 & 1 & 1 & 1 & 1 & 1 & 1 & 1 & 7 \\
\hline$\%$ Exotic grass, herb, or rush & 1 & 3 & 3 & 7 & 7 & 1 & 6 & 4 & 3 & 1 \\
\hline $\begin{array}{r}\text { Elapse Time } \\
\end{array}$ & 10 & 4 & 5 & 6 & $x$ & 4 & 4 & 10 & 4 & 8. \\
\hline Stand Age & 3 & 2 & 1.5 & $x$ & 2 & 2 & 2 & 2 & 2 & 4 \\
\hline trees, present/absent & 1 & 1 & 1 & 1 & 1 & 1 & 1 & 1 & 1 & 2 \\
\hline trees, percent cover & 1 & 1 & 1 & 1 & 1 & 1 & 1 & 1 & 1 & 4 \\
\hline trees, average dbh & 1 & 1 & 1 & 1 & 1 & 1 & 1 & 1 & 1 & 4 \\
\hline trees, max dbh & 1 & 1 & 1 & 1 & 1 & 1 & 1 & 1 & 1 & 7 \\
\hline trees, height & 1 & 1 & 1 & 1 & 1 & $\mathrm{I}$ & 1 & 1 & 1 & 4 \\
\hline basal area & 1 & 1 & 1 & 1 & 1 & 1 & 1 & 1 & 1 & 4 \\
\hline snags, 4-6"dbh/ac & 1 & 1 & 1 & 1 & 1 & 1 & 1 & 1 & 1 & 4 \\
\hline snags, 7-9"dbh/ac & 1 & 1 & 1 & 1 & 1 & 1 & 1 & 1 & $!$ & 4 \\
\hline snags, $>10^{\prime \prime} \mathrm{dbh} / \mathrm{ac}$ & 1 & 1 & 1 & 1 & 1 & 1 & 1 & 1 & 1 & 3 \\
\hline shrubs, percent cover & 1 & 1 & 1 & 1 & 1 & 1 & 1 & 1 & 1 & 4 \\
\hline shrubs, \% deciduous cover & 1 & 1 & 1 & 1 & 1 & 1 & 1 & 1 & 1 & 7 \\
\hline shrubs, \% hydrophytic cover & 1 & 1 & 1 & 1 & $\mathrm{l}$ & 1 & 1 & 1 & 1 & 7 \\
\hline shrubs, average height & 1 & 1 & 1 & 1 & 1 & 1 & 1 & 1 & 1 & 5 \\
\hline grass-herb, percent cover & 6 & 1 & 1 & 6 & 6 & 1 & 7 & 1 & $\mathrm{I}$ & 7 \\
\hline grass, percent cover & 6 & 1 & 5 & 6 & 6 & 1 & 7 & 1 & 1 & 7 \\
\hline grass as $\%$ of herb \& grass & 6 & 1 & 6 & 3 & 3 & 1 & 7 & 1 & 1 & 7 \\
\hline 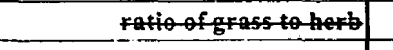 & 6 & 1 & 1 & 1 & 1 & 1 & 1 & 1 & 1 & 1 \\
\hline herbs, average height & 2 & 1 & 2 & 2 & 2 & 1 & 2 & 1 & 1 & 4 \\
\hline herbs, percent cover $<2 \mathrm{ft}$ & 5 & 1 & 6 & 3 & 7 & 1 & 6 & 1 & 1 & 2 \\
\hline SGCM, \% organic cover & 1 & 1 & 1 & 1 & 1 & 1 & 1 & 1 & 1 & 1 \\
\hline PEM, percent cover & 1 & 4 & 1 & 1 & 1 & 1 & 1 & 4 & 5 & 1 \\
\hline PAB4, percent cover & 1 & 1 & 1 & 1 & 1 & 1 & 1 & 1 & 1 & 1 \\
\hline human disturbance, distance & 3 & 2 & 3 & 3 & 3 & 5 & 4 & 3 & 6 & 5 \\
\hline quail, distance escape cover & 1 & 1 & 1 & 1 & 1 & 1 & 1 & 1 & 1 & 1 \\
\hline quail, diameter escape patches & 3 & 1 & 3 & 3 & 4 & 1 & 4 & 1 & 1 & 1 \\
\hline quail, distance between patches & 1 & 1 & 1 & 1 & 2 & 1 & 2 & 1 & 1 & 1 \\
\hline goose, nesting habitat & 1 & 1 & 1 & 1 & 1 & 1 & 1 & 1 & 1 & 1 \\
\hline goose, distance nest/forage & 3 & 1 & 3 & 1 & 2 & 1 & 4 & 1 & 1 & 1 \\
\hline mallard, dist. nestrearing & 1 & 1 & 1 & 1 & 2 & 2 & 2 & 1 & 2 & $i$ \\
\hline mallard, height residual cover & 1 & 1 & 1 & 2 & 1 & 1 & 2 & 1 & 1 & 1 \\
\hline mallard, percent nest cover & 6 & 1 & 3 & 3 & 1 & 1 & 3 & 1 & 1 & 1 \\
\hline mallard, ratio PEM to POW & 1 & 1 & 1 & 1 & 1 & 1 & 1 & 2 & 1 & 1 \\
\hline mallard, water regime & 1 & 3 & 1 & 1 & 1 & 2 & 1 & 3 & 3 & 6 \\
\hline sandpiper, $\%$ organic cover & 1 & 1 & 1 & 1 & 1 & 1 & 1 & 1 & 1 & 1 \\
\hline sandpiper, distance nest/water & 1 & 1 & 1 & 1 & 1 & 1 & 1 & 1 & 1 & 1 \\
\hline mink, $\%$ year with water & 1 & 4 & 1 & 1 & 1 & 5 & 1 & 5 & 5 & 5 \\
\hline mink, \% tree/shrub cover 330ft & 1 & 1 & 1 & 1 & 1 & 1 & 1 & 3 & 4 & 5 \\
\hline mink, \% tree/shrub cover edge & 1 & 1 & 1 & 1 & 1 & 1 & 1 & 3 & 4 & 5 \\
\hline meadowlark, dist. perches & 1 & 1 & 2 & 2 & 2 & 1 & 2 & 1 & 1 & 1 \\
\hline heron, dist. nest/foraging & 4 & 4 & 4 & 6 & 4 & 5 & 5 & 4 & 6 & 3 \\
\hline heron, forage quality & 1 & 4 & 1 & 1 & 2 & 4 & 2 & 4 & 3 & 5 \\
\hline heron, potential nest sites & 1 & 1 & 1 & 1 & 1 & 1 & 1 & 1 & 1 & 2 \\
\hline heron, distance potential nests & 6 & 6 & 4 & 6 & 1 & 7 & 7 & 7 & 6 & 2 \\
\hline
\end{tabular}


- Table C-6. Yakama Method Field Estimates, Ordered by Plot Serial Number.

\begin{tabular}{|c|c|c|c|c|c|c|c|c|c|c|}
\hline & & & & & & & $!$ & 1 & 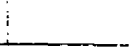 & \\
\hline & & & & & & & 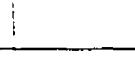 & 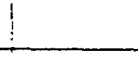 & & \\
\hline & & & & & & & & 1 & & \\
\hline Serial Number & 11 & 12 & 13 & 14 & 15 & 16 & 17 & 18 & 19 & 20 \\
\hline Plot Number & SLA-11 & SLA-12 & SLA-13 & SLA-14 & TP-1 & TP-2 & TP-3 & $\mathrm{TP}-4$ & IP-5 & TP-6 \\
\hline Date & $8 / 9 / 99$ & $8 / 9 / 99$ & $8 / 9 / 99$ & $8 / 9 / 99$ & $8 / 10 / 99$ & $8 / 10 / 99$ & $8 / 10 / 99$ & $8 / 10 / 99$ & $8 / 10 / 99$ & $8 / 10 / 99$ \\
\hline Observers & $\mathrm{TH}, \mathrm{A}, \mathrm{H}$ & $\mathrm{TH}, \mathrm{A}, \mathrm{H}$ & $\mathrm{TH}, \mathrm{A}, \mathrm{H}$ & all & all & all & all & all & all & all \\
\hline Wildlife Area & S Lat A & S Lat A & S Lat A & S Lat A & Top Pmp & Top Pmp & Top Pmp & Top Pmp & Top Pmp & Top Pmp \\
\hline 1991 Cover Type & $\underline{\mathbf{R}}$ & PEM & PEM & SSG & $\mathrm{Ag}$ & $\mathbf{R}$ & $\mathbf{A g}$ & PEM & rs & $\mathrm{Ag}$ \\
\hline 1999 Cover Type & $\underline{\mathbf{R}}$ & PEM & PEM & SSG & $\mathrm{Ag}$ & $\mathbf{R}$ & $\mathbf{A g}$ & PEM & rs & $\mathrm{Ag}$ \\
\hline RAI Cover Type & $\underline{\mathbf{R}}$ & PEM & PEM & $\mathbf{G}$ & Ag-pf & $\mathbf{R}$ & Ag-pf & PEM & $\underline{s}$ & Ag-pf \\
\hline$\%$ Exotic Shrubs & 6 & 1 & 1 & 1 & 1 & 1 & 1 & 1 & 4 & -1 \\
\hline$\%$ Exotic grass, berb, or rush & 1 & 3 & 2 & 1 & 7 & 1 & 7 & 3 & 1 & 7 \\
\hline Elapse Time & 4 & 23 & $x$ & 5 & 5 & 4 & $x$ & 4 & $x$ & $x$ \\
\hline Stand Age & 4 & 3 & 3 & 3 & $\mathrm{x}$ & $x$ & 3 & 3 & 5 & 3 \\
\hline trees, present/absent & 1 & 1 & 1 & 1 & 1 & 1 & 1 & 1 & 1 & 1 \\
\hline trees, percent cover & 1 & 1 & 1 & 1 & 1 & 1 & 1 & 1 & 1 & 1 \\
\hline trees, average dbh & 1 & 1 & 1 & 1 & 1 & 1 & 1 & 1 & 1 & 1 \\
\hline trees, $\max \mathrm{dbh}$ & 1 & 1 & 1 & 1 & 1 & 1 & 1 & 1 & 1 & 1 \\
\hline trees, height & 1 & 1 & 1 & 1 & 1 & 1 & 1 & 1 & 1 & 1 \\
\hline basal area & 1 & 1 & 1 & 1 & 1 & 1 & 1 & 1 & 1 & 1 \\
\hline snags, 4-6"dbh/ac & 1 & 1 & 1 & 1 & 1 & 1 & 1 & 1 & 1 & 1 \\
\hline snags, 7-9"dbh/ac & 1 & 1 & 1 & 1 & 1 & 1 & 1 & 1 & 1 & 1 \\
\hline snags, $>10^{\prime \prime} \mathrm{dbh} / \mathrm{ac}$ & 1 & 1 & 1 & 1 & 1 & 1 & 1 & 1 & 1 & 1 \\
\hline shrubs, percent cover & 1 & 1 & 1 & 1 & 1 & 1 & 1 & 1 & 6 & 4 \\
\hline shrubs, $\%$ deciduous cover & 1 & 1 & 1 & 1 & 1 & 1 & 1 & 1 & 6 & 4 \\
\hline shrubs, $\%$ hydrophytic cover & 1 & 1 & 1 & 1 & 1 & 1 & 1 & 1 & 6 & $i$ \\
\hline shrubs, average height & 1 & 1 & 1 & 1 & 1 & 1 & 1 & 1 & 6 & 6 \\
\hline grass-herb, percent cover & 1 & 1 & 1 & 7 & 7 & 1 & 7 & 1 & 4 & 7 \\
\hline grass, percent cover & 1 & 1 & 1 & 5 & 5 & 1 & 5 & 1 & 4 & 7 \\
\hline grass as $\%$ of herb \& grass & 1 & 1 & 1 & 5 & 5 & 1 & 5 & 1 & 7 & 7 \\
\hline Fatoof gross to heth & 1 & 1 & 1 & 1 & 1 & 1 & 1 & 1 & 1 & 1 \\
\hline herbs, average beight & 1 & 1 & 1 & 2 & 3 & 1 & 3 & 1 & 4 & 3 \\
\hline herbs, percent cover $<2$ it & 1 & 1 & 1 & 7 & 6 & 1 & 4 & 1 & 1 & 4 \\
\hline SGCM, \% organic cover & 1 & 1 & 1 & 1 & 1 & 1 & 1 & 1 & i & 1 \\
\hline PEM, percent cover & 3 & 6 & 5 & 1 & 1 & 1 & 1 & 5 & 1 & 1 \\
\hline PAB4, percent cover & 2 & 3 & 1 & 1 & 1 & 1 & 1 & 1 & 1 & 1 \\
\hline human disturbance, distance & 5 & 5 & 5 & 5 & 3 & 6 & 5 & 5 & 5 & 5 \\
\hline quail, distance escape cover & 1 & 1 & 1 & 3 & 3 & 1 & 1 & 1 & 2 & 2. \\
\hline quail, diameter escape patches & 1 & 1 & 1 & 4 & 2 & 1 & 1 & 1 & 4 & 4 \\
\hline quail, distance between patches & 1 & 1 & 1 & 4 & 4 & 1 & 1 & 1 & 3 & 4 \\
\hline goose, nesting habitat & 1 & 1 & 1 & 1 & 1 & 1 & 1 & 1 & 1 & 1 \\
\hline goose, distance nest/forage & 1 & 1 & 1 & 3 & 2 & 1 & 2 & 2 & 6 & 2 \\
\hline mallard, dist. nest/rearing & 2 & 2 & 2 & 2 & 3 & 2 & 2 & 2 & 2 & 3 \\
\hline mallard, height residual cover & 1 & 1 & 1 & 2 & 3 & 1 & 4 & 1 & 5 & 3 \\
\hline mallard, percent nest cover & $i$ & 1 & 1 & 3 & 4 & 1 & 7 & 1 & 5 & 7 \\
\hline mallard, ratio PEM to POW & $!$ & 3 & 2 & 1 & 1 & 3 & 1 & 3 & 1 & 1 \\
\hline mallard, water regime & 2 & 3 & 3 & 1 & 1 & 2 & 1 & 3 & 6 & 1 \\
\hline sandpiper, $\%$ organic cover & 1 & 1 & 1 & 1 & 1 & 1 & 1 & 1 & 1 & 1 \\
\hline sandpiper, distance nest/water & 1 & 1 & 1 & 1 & 1 & 1 & 1 & 1 & 1 & 1 \\
\hline mink, $\%$ year with water & 5 & 5 & 5 & 1 & 1 & 5 & 1 & 5 & 5 & 1 \\
\hline mink, $\%$ tree/shrub cover $330 \mathrm{ft}$ & 4 & 5 & 3 & 1 & 1 & 4 & 1 & 4 & 7 & 1 \\
\hline mink, $\%$ tree/shrub cover edge & 4 & 5 & 3 & 1 & 1 & 4 & 1 & 4 & 7 & 1 \\
\hline meadowlark, dist. perches & 1 & 1 & 1 & 3 & 3 & 1 & 2 & 1 & 1 & 3 \\
\hline heron, dist. nest/foraging & 6 & 7 & 5 & 5 & 5 & 6 & 5 & 6 & 1 & 6 \\
\hline heron, forage quality & 4 & 5 & 4 & 2 & 2 & 4 & 2 & 5 & 1 & 2 \\
\hline heron, potential nest sites & 1 & 1 & 1 & 1 & 1 & 1 & 1 & 1 & 1 & 1 \\
\hline heron, distance potential nests & 6 & 7 & 3 & 5 & 5 & 6 & 5 & 6 & 5 & 6 \\
\hline
\end{tabular}


- Table C-6. Yakama Method Field Estimates, Ordered by Plot Serial Number.

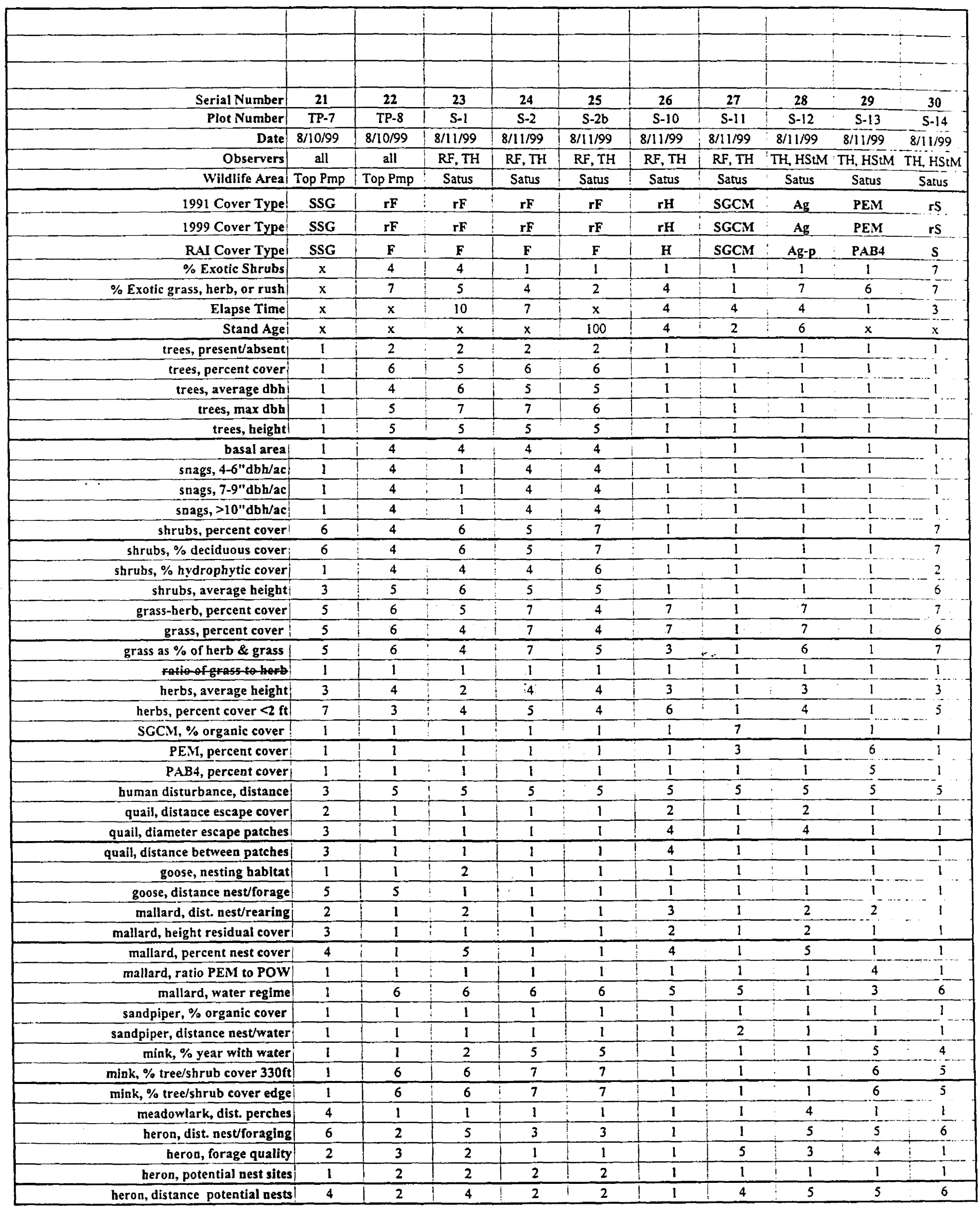


- Table C-6. Yakama Method Field Estimates, Ordered by Plot Serial Number.

\begin{tabular}{|c|c|c|c|c|c|c|c|c|c|c|}
\hline & & & & & 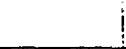 & 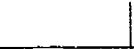 & 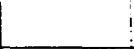 & 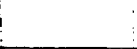 & & 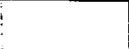 \\
\hline & & & & & & & & & 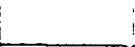 & $\vdots$ \\
\hline & & & & & & & & & $\vdots$ & \\
\hline Serial Number & 31 & 32 & 33 & 34 & 35 & 36 & 37 & 38 & 39 & 40 \\
\hline Plot Number & NS-1 & NS-2 & NS-11 & NS-12 & NS-13 & NS-14 & SS-1 & SS-2 & SS-3 & $S S-4$ \\
\hline Date & $8 / 12 / 99$ & $8 / 12 / 99$ & $8 / 12 / 99$ & $8 / 12 / 99$ & $8 / 12 / 99$ & $8 / 12 / 99$ & $8 / 12 / 99$ & $8 / 12 / 99$ & $8 / 12 / 99$ & $8 / 12 / 99$ \\
\hline Observers & $\mathrm{RS}, \mathrm{JSF}$ & $R F, J S F$ & RF, JSF & TH, Feen & $\mathrm{TH}$, Feen & TH, Feen & TH RF JS & THRF JS & THRF JS & TH RF JS \\
\hline Wildlife Area & N-Satus & N-Satus & N-Satus & N-Satus & N-Satus & N-Satus & S-Satus & S-Satus & S.Satus & S-Satus \\
\hline 1991 Cover Type & SSG & $\mathrm{Ag}$ & SSG & $\mathbf{r F}$ & SGCM & $\mathbf{R}$ & PEM & SSG & SSG & PEM \\
\hline 1999 Cover Type & SSG & $\mathrm{Ag}$ & SSG & $r F$ & SGCM & $\mathbf{R}$ & PEM & SSG & SSG & PEM \\
\hline RAI Cover Type & SSG & Ag-pf & SSG & $\mathbf{F}$ & SGCM & $\mathbf{R}$ & PEM & SSG & $\mathbf{G}$ & PAB4 \\
\hline \% Exotic Shrubs & $\mathrm{x}$ & 1 & 1 & 1 & 11 & 3 & 1 & 1 & 1 & 1 \\
\hline$\%$ Exotic grass, herb, or rush & 3 & 6 & 6 & 7 & 3 & 1 & 2 & 1 & 6 & 3 \\
\hline Elapse Time & $x$ & 6 & 7 & 12 & 2 & 1 & 5 & $x$ & 7 & 7 \\
\hline Stand Age & $x$ & $x$ & $x$ & $x$ & 2 & $x$ & 7 & $x$ & $x$ & $\mathrm{x}$ \\
\hline trees, present/absent & 1 & 1 & 1 & 2 & 1 & 1 & 1 & 1 & 1 & 1 \\
\hline trees, percent cover & 1 & 1 & 1 & 5 & 1 & 1 & 1 & 1 & 1 & $!$ \\
\hline trees, average dbhi & 1 & 1 & 1 & 5 & 1 & 1 & 1 & 1 & 1 & 1 \\
\hline trees, $\max \mathrm{dbh}$ & 1 & 1 & 1 & 6 & 1 & 1 & 1 & 1 & 1 & 1 \\
\hline trees, height & 1 & 1 & 1 & 5 & 1 & 1 & 1 & 1 & 1 & 1 \\
\hline basal areal & 1 & 1 & 1 & 4 & 1 & 1 & 1 & $i$ & 1 & 1 \\
\hline snags, 4-6"dbh/ac & 1 & 1 & 1 & 5 & 1 & 1 & 1 & 1 & 1 & 1 . \\
\hline snags, 7.9"dbh/ac & 1 & 1 & 1 & 5 & 1 & 1 & 1 & 1 & 1 & 1. \\
\hline snags, $>10 " \mathrm{dbh} / \mathrm{ac}$ & 1 & 1 & 1 & 5 & 1 & 1 & 1 & 1 & 1 & 1 \\
\hline shrubs, percent cover & 4 & 3 & 5 & 5 & 1 & 1 & 1 & 5 & 2 & 1 \\
\hline shrubs, $\%$ deciduous cover & 1 & 1 & 1 & 5 & 1 & 1 & 1 & 1 & 2 & $\mathrm{l}$ \\
\hline shrubs, \% hydrophytic cover & I & 1 & 1 & 2 & 1 & 1 & 1 & 1 & 1 & 1 \\
\hline shrubs, average height| & 3 & 3 & 3 & 4 & 1 & 1 & 1 & 3 & 4 & 1 \\
\hline grass-herb, percent cover & 4 & 7 & 4 & 6 & 3 & 1 & 1 & 5 & 7 & 1 \\
\hline grass, percent cover & 4 & 7 & 4 & 6 & 1 & 1 & 1 & 5 & 7 & 1 \\
\hline grass as $\%$ of herb \& grass & 5 & 3 & 7 & 7 & 1 & 1 & 1 & 5 & 5 & 1 \\
\hline 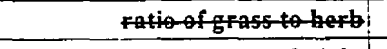 & 1 & 1 & 1 & 1 & 1 & 1 & 1 & 1 & 1 & 1 \\
\hline herbs, average height & 2 & 3 & 2 & 2 & 2 & 1 & 1 & 3 & 3 & 1 \\
\hline herbs, percent cover $<2 \mathrm{ft}$ & 6 & 5 & 7 & 3 & 3 & 1 & 1 & 7 & 6 & 1 \\
\hline SGCM, \% organic cover & 1 & 1 & 1 & 1 & 3 & 1 & $i$ & 1 & 1 & 1 \\
\hline PEM, percent cover : & 1 & 1 & 1 & 1 & 1 & 2 & 5 & 1 & 1 & 3 \\
\hline PAB4, percent cover & 1 & 1 & 1 & 1 & 1 & 2 & 5 & 1 & 1 & 4 \\
\hline human disturbance, distance & 5 & 5 & 5 & 5 & 5 & 5 & 5 & 5 & 4 & 5 \\
\hline quail, distance escape cover & 4 & 5 & 5 & 1 & 1 & 1 & 1 & 2 & 5 & 1. \\
\hline quail, diameter escape patches & 3 & 4 & 4 & 1 & 1 & 1 & 1 & 2 & 2 & 1 \\
\hline quail, distance betrveen patches & 5 & 5 & 6 & 1 & 1 & 1 & 1 & 2 & 6 & 1 \\
\hline goose, nesting babitat & 1 & 1 & 1 & 3 & 1 & 1 & 1 & 1 & 1 & 1 \\
\hline goose, distance nest/forage & 1 & 1 & 3 & 2 & 1 & 2 & 1 & 1 & 2 & 1 \\
\hline mallard, dist. nestrearing & 2 & 2 & 3 & 1 & 1 & 1 & 2 & 2 & 2 & 2 \\
\hline mallard, height residual cover & 4 & 3 & 3 & 1 & 1 & 1 & 1 & 3 & 2 & 1 \\
\hline mallard, percent nest cover & 3 & 4 & 4 & 1 & 1 & 1 & 1 & 4 & 3 & $!$ \\
\hline mallard, ratio PEM to POW & 1 & 1 & 1 & 1 & 1 & 1 & 3 & 1 & 1 & 2 \\
\hline mallard, water regime & 1 & 1 & 1 & 6 & 5 & 2 & 3 & 1 & 1 & 3 \\
\hline sandpiper, \% organic cover & 1 & 1 & 1 & 1 & 1 & 1 & 1 & 1 & 1 & 1 \\
\hline sandpiper, distance nest/water & 1 & 1 & 1 & 1 & 2 & 1 & 1 & 1 & 1 & 1 \\
\hline mink, $\%$ year with water & 1 & 1 & 1 & 3 & 1 & 5 & 5 & 1 & 1 & 5 \\
\hline mink, $\%$ tree/shrub cover $330 \mathrm{ft}$ & 1 & 1 & 1 & 6 & 1 & 5 & 4 & 1 & 1 & 6 \\
\hline mink, $\%$ tree/shrub cover edge & 1 & 1 & 1 & 6 & 1 & 5 & 4 & 1 & 1 & 6 \\
\hline meadowlark, dist. perches & 4 & 3 & 3 & 1 & 1 & 1 & 1 & 3 & 2 & 1 \\
\hline heron, dist. nest/foraging & 1 & 5 & 3 & 1 & 3 & 5 & 6 & 6 & 7 & 1 \\
\hline heron, forage quality & 1 & 2 & 2 & 1 & 1 & 5 & 5 & 2 & 2 & 5 \\
\hline beron, potential nest sites & 1 & 1 & 1 & 2 & 1 & 1 & 1 & 1 & 1 & 1 \\
\hline beron, distance potential nests & 1 & 5 & 3 & 4 & 3 & 6 & 6 & 5 & 7. & 6 \\
\hline
\end{tabular}


-Table C-6. Yakama Method Field Estimates, Ordered by Plot Serial Number.

\begin{tabular}{|c|c|c|c|c|c|c|c|c|c|c|}
\hline & & & & & & & & $\vdots$ & & \\
\hline & & & & & & & & 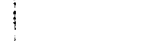 & & \\
\hline & & & & & & & & i & & \\
\hline Serial Number & 41 & 42 & 43 & 44 & 45 & 46 & 47 & 48 & 49 & 50 \\
\hline Plot Number & SS-5 & SS-5 & SS-6 & WS-1 & WS-2 & WS-3 & WS- 4 & WS-5 & WS-6 & $\mathrm{M}-1$ \\
\hline \begin{tabular}{r|r|} 
Date & 8 \\
\end{tabular} & $8 / 12 / 99$ & $8 / 12 / 99$ & $8 / 12 / 99$ & $8 / 13 / 99$ & $8 / 13 / 99$ & $8 / 13 / 99$ & $8 / 13 / 99$ & $8 / 13 / 99$ & $8 / 13 / 99$ & $8 / 13 / 99$ \\
\hline Observers I & TH RS JS & TH RF JS & TH RF JS & TH RF LJ & TH RF LJ & TH RF L J & TH RF LJ & $1 \mathrm{THRF} \mathrm{LJ}$ & TH RF L & TH RF LJ \\
\hline Wildlife Area & S-Satus & S-Satus & S-Satus & Wanity & Wanity & Wanity & Wanity & Wanity & Wanity & Mosebar \\
\hline 1991 Cover Type & $\mathrm{Ag}$ & SGCM & $\mathbf{R}$ & $\mathbf{A g}$ & $\mathrm{Ag}$ & $\mathrm{Ag}$ & $\mathbf{R}$ & $\mathrm{rS}$ & $\mathrm{Ag}$ & SGCM \\
\hline 1999 Cover Type & $\mathrm{Ag}$ & SGCM & $\mathbf{R}$ & $\mathrm{Ag}$ & $\mathrm{Ag}$ & $\mathrm{Ag}$ & $\mathbf{R}$ & rs & $\mathrm{Ag}$ & SGCM \\
\hline RAI Cover Type & Ag-p & SGCM & $\mathbf{R}$ & Ag-p & Ag-p & Ag-pf & $\mathbf{R}$ & $\mathbf{s}$ & Ag-p & SGCM \\
\hline$\%$ Exotic Shrubs & 1 & 1 & 1 & 2 & 2 & 1 & 1 & 2 & 1 & $x$ \\
\hline$\%$ Exotic grass, herb, or rush & 7 & 1 & 4 & 2 & 2 & 2 & 2 & 2 & 2 & $\mathrm{x}$ \\
\hline Elapse Time & $x$ & $x$ & 2 & 5 & 3 & 4 & 2 & 5 & 4 & 9 \\
\hline Stand Age! & $x$ & 2 & 2 & $x$ & $x$ & $x$ & 2 & $x$ & $x$ & 2 \\
\hline trees, present/absent & 1 & 1 & 1 & 1 & 1 & 1 & 1 & 1 & 1 & $!$ \\
\hline trees, percent cover & 1 & 1 & 1 & 1 & 1 & 1 & 1 & I & 1 & 1. \\
\hline trees, average dbh & 1 & 1 & 1 & 1 & 1 & 1 & 1 & 1 & 1 & 1 \\
\hline trees, max dbh & 1 & 1 & 1 & 1 & 1 & 1 & 1 & 1 & 1 & 1 \\
\hline trees, height & 1 & 1 & 1 & 1 & 1 & 1 & 1 & 1 & 1 & 1 \\
\hline basal area & 1 & 1 & 1 & 1 & 1 & 1 & 1 & 1 & 1 & 1 \\
\hline snags, 4-6"dbh/ac & 1 & 1 & 1 & 1 & 1 & 1 & 1 & 1 & 1 & 1 \\
\hline snags, 7.9 "dbh/ac & 1 & 1 & 1 & 1 & 1 & 1 & 1 & 1 & 1 & 1 \\
\hline snags, $>10 " \mathrm{dbh} / \mathrm{ac}$ & 1 & 1 & 1 & 1 & 1 & 1 & 1 & 1 & 1 & 1 \\
\hline shrubs, percent cover! & 1 & 1 & 1 & 4 & 4 & 4 & 1 & 6 & 1 & + \\
\hline shrubs, $\%$ deciduous cover & 1 & 1 & 1 & 1 & 1 & 4 & 1 & 6 & 1 & 1 \\
\hline shrubs, $\%$ hydrophytic cover & 1 & 1 & 1 & 1 & 1 & $\mathrm{I}$ & 1 & 5 & 1 & 1 \\
\hline shrubs, average height & 1 & 1 & 1 & 3 & 3 & 3 & 1 & 5 & 1 & 3 \\
\hline grass-herb, percent cover & 7 & 1 & 1 & 6 & 6 & 7 & 1 & 4 & 7 & 5 \\
\hline grass, percent cover & 7 & 1 & 1 & 6 & 6 & 7 & 1 & 4 & 7 & 5 \\
\hline grass as $\%$ of berb \& grass & 5 & 1 & 1 & 6 & 6 & 4 & 1 & 5 & 6 & 4 \\
\hline C L of grass to herb & 1 & 1 & 1 & 1 & 1 & 1 & 1 & 1 & 1 & 1 \\
\hline herbs, average height & 2 & 1 & 1 & 2 & 2 & 3 & 1 & 2 & 2 & 2 \\
\hline herbs, percent cover $<2 \mathrm{ft}$ & 7 & 1 & 1 & 7 & 7 & 7 & 1 & 6 & 7 & 7 \\
\hline SGCMI, \% organic cover & 1 & 1 & 3 & 1 & 1 & 1 & 1 & 1 & 1 & 1 \\
\hline PEM, percent cover: & 1 & 1 & 1 & 1 & 1 & 1 & 3 & 1 & 1 & 1 \\
\hline PAB4, percent cover & 1 & 1 & 1 & 1 & 1 & 1 & 1 & 1 & 1 & 1 \\
\hline human disturbance, distance & 5 & 6 & 5 & 5 & 5 & 5 & 5 & 5 & 5 & 5 \\
\hline quail, distance escape cover & 3 & 1 & 1 & 5 & 5 & 2 & 1 & 2 & 2 & 3 \\
\hline quail, diameter escape patches & 3 & 1 & 1 & 3 & 3 & 3 & 1 & 4 & 3 & 3 \\
\hline quail, distance between patches & 4 & 1 & 1 & 6 & 6 & 4 & 1 & 3 & 3 & 4 \\
\hline goose, nesting habitat & 1 & 1 & 1 & 1 & 1 & 1 & 1 & 1 & 1 & 1 \\
\hline goose, distance nest/forage & 2 & 1 & 1 & 1 & 1 & 1 & 1 & 1 & 1 & 1 \\
\hline mallard, dist. nest/rearing & 2 & 1 & 1 & 2 & 2 & 2 & 1 & 2 & 1 & $i$ \\
\hline mallard, height residual cover! & 5 & 1 & 1 & 2 & 2 & 3 & 1 & 3 & 1 & 2 \\
\hline mallard, percent nest cover & 3 & 1 & 1 & 2 & 2 & 4 & 1 & 4 & 1 & 4 \\
\hline mallard, ratio PEM to POW! & 1 & 1 & 1 & 1 & 1 & 1 & 1 & 1 & 1 & 1 \\
\hline mallard, water regime & 1 & 5 & 2 & 1 & 1 & $\mathrm{I}$ & 2 & 6 & 1 & 5 \\
\hline sandpiper, $\%$ organic cover & $i$ & 1 & 1 & 1 & 1 & 1 & 1 & 1 & 1 & 1 \\
\hline sandpiper, distance nest/water & 1 & 2 & 1 & 1 & 1 & 1 & 1 & 1 & 1 & 1 \\
\hline mink, $\%$ year with water & 1 & 1 & 5 & 1 & 1 & 1 & 5 & 5 & 1 & 1 \\
\hline mink, \% tree/shrub cover 330ft & 1 & 1 & 6 & 1 & 1 & 1 & 5 & 4 & 1 & 1 \\
\hline mink, \% tree/shrub cover edge & 1 & 1 & 6 & 1 & 1 & 1 & 5 & 5 & 1 & 1 \\
\hline meadowlark, dist. perches & 3 & 1 & 1 & 2 & 2 & 3 & 1 & 2 & 2 & 2 \\
\hline heron, dist. nest/foraging & 6 & 1 & 6 & 7 & 7 & 6 & 5 & 5 & 5 & 4 \\
\hline heron, forage quality & 2 & 1 & 5 & 2 & 2 & 2 & 4 & 2 & 2 & 2 \\
\hline heron, potential nest sites: & 1 & 1 & 1 & 1 & 1 & 1 & 1 & 1 & 1 & 1 \\
\hline heron, distance potential nests & 6 & 1 & 6 & 7 & 7 & 6 & 5 & 5 & 5 & 4 \\
\hline
\end{tabular}


'Table C-6. Yakama Method Field Estimates, Ordered by Plot Serial Number.

\begin{tabular}{|c|c|c|c|c|c|c|c|c|c|c|}
\hline & & & & & & & $i$ & ! & & \\
\hline & & & & & & & 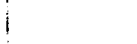 & i & & \\
\hline & & & & & & & & & & \\
\hline Serial Number & 51 & 52 & 53 & 54 & 55 & 56 & 57 & 58 & 59 & 60 \\
\hline Plot Number & $M-2$ & M-3 & $M-4$ & M.5 & $M-6$ & M-8 & M-9 & M-10 & $M \cdot 1 \mathrm{I}$ & S3-1 \\
\hline \begin{tabular}{r|r} 
Date & 8 \\
\end{tabular} & $8 / 13 / 99$ & $8 / 13 / 99$ & $8 / 13 / 99$ & $8 / 17 / 99$ & $8 / 17 / 99$ & $8 / 17 / 99$ & $8 / 17 / 99$ & $8 / 17 / 99$ & $8 / 17 / 99$ & $8 / 17 / 99$ \\
\hline $\begin{array}{ll}\text { Observers } T \\
\end{array}$ & TH RF LJ & TH RF U & THRF LJ & $\mathrm{TH}, \mathrm{RF}$ & TH, RF & $\mathrm{TH}, \mathrm{RF}$ & $\mathrm{TH}, \mathrm{RF}$ & $\mathrm{TH}, \mathrm{RF}$ & $\mathrm{TH}, \mathrm{RF}$ & $\mathrm{TH}, \mathrm{RF}$ \\
\hline \begin{tabular}{|l|l} 
Wildlife Area \\
\end{tabular} & Mosebar & Mosebar & Mosebar & Mosebar & Mosebar & Mosebar & Mosebar & Mosebar & Mosebar & Satus \\
\hline 1991 Cover Type & PEM & Ag & $\mathbf{L}$ & $\mathrm{Ag}$ & $\mathrm{Ag}$ & $\mathbf{r H}$ & rS & $\mathrm{Ag}$ & $\mathbf{L}$ & $\mathrm{rS}$ \\
\hline 1999 Cover Type & PEM & Ag & $\underline{L}$ & $\mathrm{Ag}$ & $\mathrm{Ag}$ & $\mathrm{rH}$ & rS & $\mathrm{Ag}$ & $\mathbf{L}$ & rS \\
\hline RAI Cover Type & PEM & Ag-pf & $\mathrm{L}$ & Ag-pf & $A g-C$ & $\mathbf{H}$ & $\underline{s}$ & Ag-pf & POW & $s$ \\
\hline$\%$ Exotic Shrubs & 1 & 1 & 1 & 2 & 1 & 1 & 2 & 1 & 1 & 1 \\
\hline$\%$ Exotic grass, herb, or rush & 2 & 7 & 2 & 7 & 7 & 1 & 4 & 7 & 2 & 3 \\
\hline Elapse Time & 3 & 4 & 4 & $\mathrm{x}$ & 2 & 5 & 5 & 3 & 3 & 4 \\
\hline Stand Age & $x$ & $x$ & $x$ & $i$ & 1 & 2 & 1 & 1 & $x$ & $x$ \\
\hline trees, present/absent & 1 & 1 & 2 & 1 & 1 & 1 & 1 & 1 & 1 & 1 \\
\hline trees, percent cover & 1 & 1 & 2 & 1 & 1 & 1 & 1 & 1 & 1 & $i$ \\
\hline trees, average dbh & 1 & 1 & 1 & 1 & 1 & 1 & 1 & 1 & 1 & 1 \\
\hline trees, max dbh & 1 & 1 & 1 & 1 & 1 & 1 & 1 & 1 & 1 & 1 \\
\hline trees, height & 1 & 1 & 1 & 1 & 1 & 1 & 1 & 1 & 1 & 1 \\
\hline basal area & 1 & 1 & 1 & 1 & $i$ & 1 & 1 & 1 & 1 & 1 \\
\hline snags, $4-6^{\prime \prime} \mathrm{dbh} / \mathrm{ac}$ & 1 & 1 & 1 & 1 & 1 & 1 & 1 & 1 & 1 & 1 \\
\hline snags, 7-9"dbb/ac: & $t$ & 1 & 1 & 1 & 1 & 1 & 1 & 1 & 1 & 1 \\
\hline snags, $>10^{\prime \prime} \mathrm{dbh} / \mathrm{ac}$ & 1 & 1 & 1 & & 1 & 1 & 1 & 1 & 1 & 1 \\
\hline shrubs, percent cover & 1 & 4 & 1 & 4 & 1 & 1 & 7 & 1 & 1 & 6 \\
\hline shrubs, $\%$ deciduous cover & 1 & 4 & 1 & 4 & 1 & 1 & 7 & 1 & 1 & 6 \\
\hline shrubs, \% hydrophytic cover & 1 & 3 & 1 & 1 & 1 & I & 4 & 1 & 1 & 5 \\
\hline shrubs, average height & 1 & 5 & 1 & 4 & 1 & 1 & 4 & 1 & 1 & 5 \\
\hline grass-herb, percent cover & 1 & 6 & 1 & 7 & 7 & 5 & 4 & 7 & i & 4 \\
\hline grass, percent cover & 1 & 6 & 1 & 7 & 7 & 5 & 4 & 7 & 1 & 4 \\
\hline grass as $\%$ of herb \& grass & 1 & 3 & 1 & 4 & 7 & 3 & $\therefore .4$ & 4 & 1 & 3 \\
\hline Fatio of grass to herb & 1 & 1 & 1 & 1 & 1 & 1 & 1 & 1 & 1 & 1 \\
\hline herbs, average height & 1 & 3 & 1 & 3 & 5 & 2 & 4 & 3 & 1 & 4 \\
\hline herbs, percent cover $<2 \mathrm{ft}$ & 1 & 4 & 1 & 7 & 1 & 7 & 3 & 7 & 3 & 5 \\
\hline SGCM, \% organic cover & 1 & 1 & 1 & 1 & 1 & 1 & 1 & 1 & 1 & 1 \\
\hline PEM, percent cover: & 6 & 1 & 4 & 1 & 1 & 1 & 1 & 1 & 4 & $i$ \\
\hline PAB4, percent cover & 1 & 1 & 2 & 1 & 1 & 1 & 1 & 1 & 1 & 1. \\
\hline human disturbance, distance & 4 & 4 & 5 & 5 & 5 & 5 & 5 & 5 & 5 & 5 \\
\hline quail, distance escape cover & 1 & 2 & 1 & 4 & 6 & 3 & 2 & 5 & 1 & 2 \\
\hline quail, diameter escape patches & 1 & 3 & 1 & 2 & 1 & 3 & 3 & 3 & 1 & 4 \\
\hline quail, distance between patchesi & 1 & 4 & 1 & 3 & 1 & 2 & 2 & 4 & 1 & 2 \\
\hline goose, nesting habitat & 1 & 1 & 1 & 1 & 1 & 1 & 1 & 1 & 1 & 1 \\
\hline goose, distance nest/forage & 1 & 2 & 1 & 2 & 1 & 2 & 2 & 2 & 1 & 1 \\
\hline mallard, dist. nest/rearing & 2 & 2 & 2 & 2 & 2 & 2 & 2 & 2 & 2 & 1 \\
\hline mallard, height residual cover & 1 & 3 & 1 & 2 & 4 & 2 & 4 & 4 & 2 & $i$ \\
\hline mallard, percent nest cover & 1 & 7 & 1 & 4 & 1 & 1 & 7 & 5 & 1 & 1 \\
\hline mallard, ratio PEM to POW & 3 & 1 & 2 & 1 & 1 & 1 & 1 & 1 & 2 & 1 \\
\hline mallard, water regime & 3 & 1 & 2 & 1 & 1 & 5 & 6 & 1 & 2 & 6 \\
\hline sandpiper, $\%$ organic cover & $i$ & 1 & 1 & 1 & 1 & 1 & 1 & 1 & 1 & 1 \\
\hline sandpiper, distance nest/water & 1 & 1 & 1 & 1 & 1 & 1 & 1 & 1 & 1 & 1 \\
\hline mink, $\%$ year with water & 5 & 1 & 1 & 1 & 1 & 1 & 5 & 1 & 1 & 1 \\
\hline mink, $\%$ tree/shrub cover $330 \mathrm{ft}$ & 4 & 1 & 1 & 1 & 1 & 1 & 6 & 1 & 1 & 1 \\
\hline mink, \% tree/shrub cover edge & 4 & 1 & 1 & 1 & 1 & 1 & 5 & 1 & 1 & 1 \\
\hline meadowlark, dist. perches & 1 & 2 & 1 & 3 & 3 & 1 & 1 & 2 & 1 & 1 \\
\hline heron, dist. nest/foraging & 5 & 3 & 3 & 4 & 1 & 4 & 4 & 4 & 1 & 1 \\
\hline heron, forage quality & 4 & 2 & 6 & 2 & 1 & 3 & 1 & 2 & 1 & 1 \\
\hline heron, potential nest sites & 1 & 1 & 1 & 1 & 1 & 1 & 1 & 1 & 1 & 1 \\
\hline heron, distance potential nests & 5 & 3 & 3 & 4 & 5 & 4 & 4 & 4 & 1 & 4 \\
\hline
\end{tabular}


. Table C-6. Yakama Method Field Estimates, Ordered by Plot Serial Number.

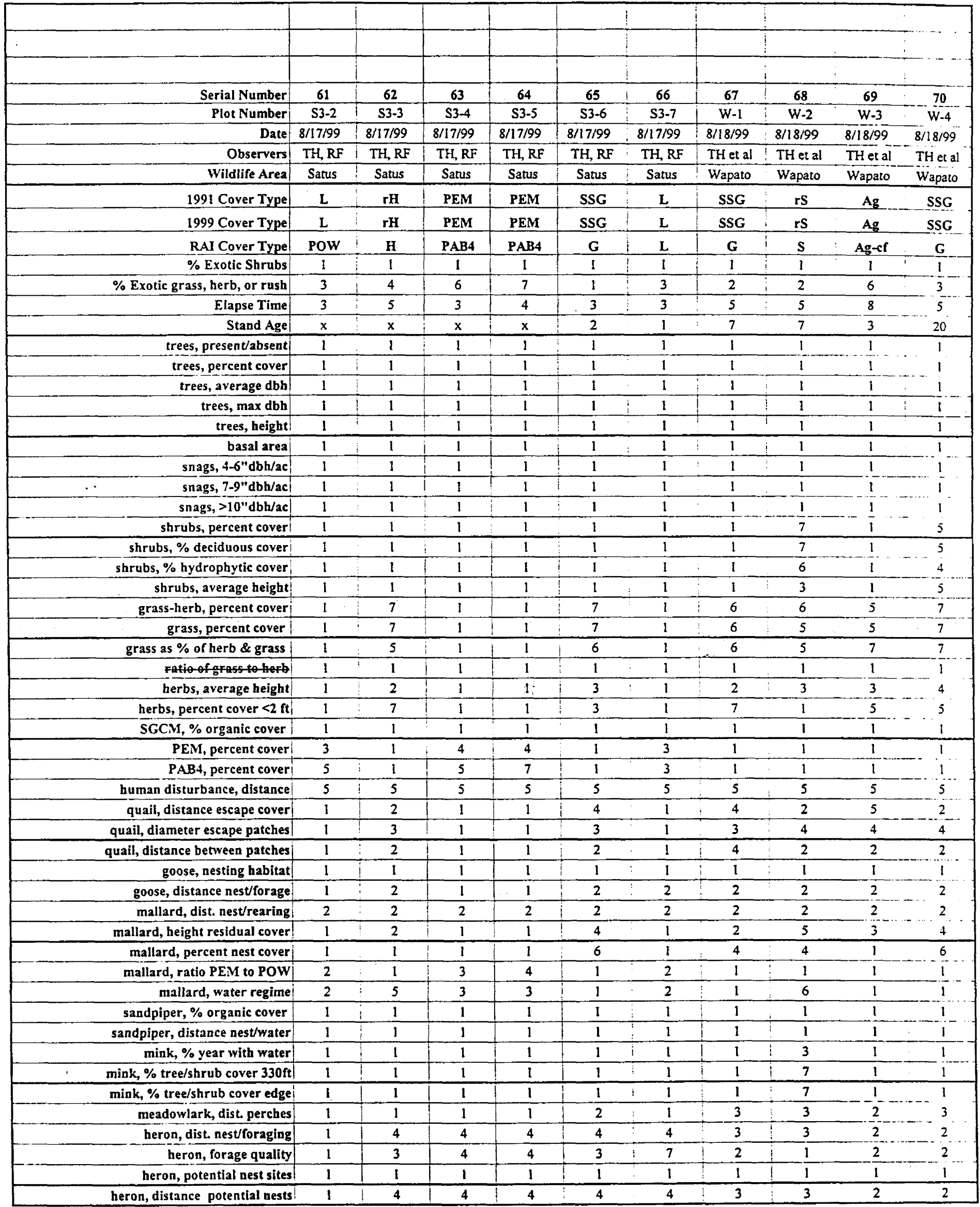


-Table C-6. Yakama Method Field Estimates, Ordered by Plot Serial Number.

\begin{tabular}{|c|c|c|c|c|c|c|c|c|c|c|}
\hline & & & & & & & 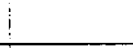 & & & \\
\hline & & & & & & & & & & \\
\hline Serial Number & 71 & 72 & 73 & 74 & 75 & 76 & 77 & 78 & 79 & 80 \\
\hline Plot Number & W-5 & W-6 & W-7 & W-8 & W-9 & LS-1 & LS-2 & LS-3 & LS-4 & LS-5 \\
\hline Date & $8 / 18 / 99$ & $8 / 18 / 99$ & $8 / 18 / 99$ & $8 / 18 / 99$ & $8 / 18 / 99$ & $8 / 19 / 99$ & $8 / 19 / 99$ & $8 / 19 / 99$ & $8 / 19 / 99$ & $8 / 19 / 99$ \\
\hline Observers & TH et al & TH et al & TH et al & Thet al & TH et al & TH et al & TH et al & THet al & THet al & THet a! \\
\hline Wildlife Area & Wapato & Wapato & Wapato & Wapato & Wapato & L-Satus & L-Satus & L-Sanus & L-Satus & L-Satus \\
\hline 1991 Cover Type & $\mathbf{r F}$ & PEM & $\mathbf{r F}$ & SSG & $\mathbf{R}$ & $\mathbf{R}$ & rs & SGCM & SSG & $\mathrm{Ag}$ \\
\hline 1999 Cover Type & $r F$ & PEM & $\mathrm{rF}$ & SSG & $\mathbf{R}$ & $\mathbf{R}$ & rs & SGCM & SSG & $\mathrm{Ag}$ \\
\hline RAI Cover Type & $\mathbf{F}$ & PEM & $\mathbf{F}$ & SSG & $\mathbf{R}$ & $\mathbf{R}$ & $S$ & SGCM & $\mathbf{G}$ & $\mathrm{Ag}-\mathrm{cf}$ \\
\hline \% Exotic Shrubs & 1 & 1 & 1 & 1 & 1 & 1 & 1 & 1 & 1 & 1 \\
\hline$\%$ Exotic grass, herb, or rush & 1 & 4 & 1 & 4 & 1 & 1 & 2 & 1 & 2 & 7 \\
\hline Elapse Time & 6 & 4 & 8 & 5 & 3 & 5 & 5 & 4 & 6 & 4 \\
\hline Stand Age & 20 & 5 & 50 & $x$ & $\mathrm{x}$ & $\mathrm{x}$ & $\mathrm{x}$ & $x$ & $x$ & 10 \\
\hline trees, present/absent & 2 & 1 & 2 & 1 & 1 & 1 & 1 & 1 & 1 & 1 \\
\hline trees, percent cover! & 7 & 1 & 6 & 1 & 1 & 1 & 1 & 1 & 1 & 1 \\
\hline trees, average dbh & 3 & 1 & 5 & 1 & 1 & 1 & 1 & 1 & 1 & 1 \\
\hline trees, $\max \mathrm{dbh}$ & 4 & 1 & 6 & 1 & 1 & 1 & 1 & 1 & 1 & 1. \\
\hline trees, height & 4 & 1 & 6 & 1 & 1 & 1 & 1 & 1 & 1 & 1 \\
\hline basal area & 4 & 1 & 4 & 1 & 1 & 1 & 1 & 1 & 1 & 1 \\
\hline snags, 4-6"dbh/ac & 4 & 1 & 3 & 1 & 1 & 1 & 1 & 1 & 1 & 1 \\
\hline snags, 7-9"dbh/aci & 4 & 1 & 2 & 1 & 1 & 1 & 1 & 1 & 1 & 1 \\
\hline snags, $>10^{\prime \prime} \mathrm{dbh} / \mathrm{ac}$ & 4 & 1 & 2 & 1 & 1 & 1 & 1 & 1 & 1 & 1 \\
\hline shrubs, percent cover & 4 & 1 & 7 & 4 & 1 & 1 & 5 & 1 & 4 & $i$ \\
\hline shrubs, $\%$ deciduous cover & 4 & 1 & 7 & 3 & 1 & 1 & 5 & 1 & 4 & 1 \\
\hline shrubs, $\%$ hydrophytic cover & 1 & 1 & 3 & 1 & 1 & 1 & 4 & 1 & 1 & 1 \\
\hline shrubs, average height! & 5 & 1 & 4 & 3 & 1 & 1 & 5 & 1 & 4 & 1 \\
\hline grass-herb, percent cover & 3 & 1 & 5 & 6 & 1 & 1 & 4 & 1 & 7 & 6 \\
\hline grass, percent cover & 3 & 1 & 4 & 6 & 1 & 1 & 5 & 1 & 7 & 6 \\
\hline grass as $\%$ of herb \& grass & 3 & 1 & 5 & 6 & 1 & 1 & - & 1 & 5 & 4 \\
\hline atio fofass to herb & 1 & 1 & 1 & 1 & 1 & 1 & 1 & 1 & 1 & 1 \\
\hline herbs, average height & 2 & 1 & 3 & 2 & 1 & 1 & 3 & 1 & 3 & 2 \\
\hline herbs, percent cover $<2 \mathrm{ft}$ & 6 & 1 & 3 & 7 & 1 & 1 & 4 & 1 & 4 & 7 \\
\hline SGCM, $\%$ organic cover & 12 & 1 & 1 & 1 & 1 & 1 & 1 & 3 & 1 & $i$ \\
\hline PEM, percent cover: & 1 & 5 & 1 & 1 & 3 & 1 & 1 & 1 & 1 & 1 \\
\hline PAB4, percent cover & 1 & 7 & 1 & 1 & 1 & 1 & 1 & 1 & 1 & 1 \\
\hline human disturbance, distance & 5 & 4 & 5 & 5 & 5 & 5 & 5 & 5 & 5 & 4 \\
\hline quail, distance escape cover & 1 & 1 & 1 & 2 & 1 & 1 & $i$ & 1 & 2 & 5 \\
\hline quail, diameter escape patches & 1 & 1 & 1 & 2 & 1 & 1 & 1 & 1 & 3 & 2 \\
\hline quail, distance between patches & 1 & 1 & 1 & 3 & 1 & 1 & 1 & 1 & 3 & 6 \\
\hline goose, nesting habitat & 1 & 1 & 2 & 1 & 1 & 1 & 1 & 1 & 1 & 1 \\
\hline goose, distance nest/forage & 1 & 2 & 2 & 2 & 2 & 4 & 4 & 4 & 4 & 4 \\
\hline mallard, dist. nest/rearing & 1 & 2 & 1 & 2 & 2 & 1 & 1 & 1 & 4 & 3 \\
\hline mallard, height residual cover & 1 & 1 & 1 & 3 & 1 & 1 & 1 & 1 & 3 & 3 \\
\hline mallard, percent nest cover & 1 & 1 & 1 & 3 & 1 & 1 & 1 & 1 & 6 & 1 \\
\hline mallard, ratio PEM to POW! & 1 & 3 & 1 & 1 & 2 & 1 & 1 & 1 & 1 & 1 \\
\hline mallard, water regime & 6 & 3 & 6 & 1 & 2 & 2 & 6 & 5 & 1 & 1 \\
\hline sandpiper, $\%$ organic cover & 1 & 1 & 1 & 1 & 1 & 1 & 1 & 1 & 1 & 1 \\
\hline sandpiper, distance nest/water & 1 & 1 & 1 & 1 & 1 & 1 & 1 & 2 & 1 & 1 \\
\hline mink, \% year with water & 3 & 3 & 3 & 1 & 5 & 5 & 5 & 1 & 1 & 1 \\
\hline mink, \% tree/shrub cover $330 \mathrm{ft}$ & 7 & 4 & 7 & 1 & 6 & 4 & 4 & 1 & $\mathrm{I}$ & 1 \\
\hline mink, $\%$ tree/shrub cover edge & 7 & 4 & 7 & 1 & 6 & 5 & 5 & 1 & 1 & 1 \\
\hline meadowlark, dist. perches & 1 & 1 & 1 & 3 & 1 & 1 & 1 & 1 & 3 & 3 \\
\hline heron, dist. nest/foraging & 3 & 2 & 2 & 2 & 2 & 7 & 7 & 7 & 6 & 6 \\
\hline heron, forage quality & 1 & 5 & 1 & 2 & 5 & 5 & 5 & 5 & 2 & 2 \\
\hline heron, potential nest sites & 1 & 1 & 2 & 1 & 1 & 1 & 1 & 1 & 1 & 1 \\
\hline heron, distance potential nests ${ }^{i}$ & 3 & 2 & 1 & 2 & 2 & 5 & 7 & 7 & 6 & 6 \\
\hline
\end{tabular}


Table C-6. Yakama Method Field Estimates, Ordered by Plot Serial Number.

\begin{tabular}{|c|c|c|c|c|c|c|c|c|}
\hline & & & & & & & 1 & 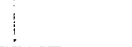 \\
\hline & & & & & & & & \\
\hline Serial Number & 81 & 82 & 83 & 84 & 85 & 86 & 87 & 88 \\
\hline Plot Number & LS-6 & LS-7 & LS-8 & LS-9 & LS-10 & LS-11 & LS-12 & LS.13 \\
\hline Date 8 & $8 / 19 / 99$ & $8 / 19 / 99$ & $8 / 19 / 99$ & $8 / 19 / 99$ & $8 / 19 / 99$ & $8 / 19 / 99$ & $8 / 19 / 99$ & $8 / 19 / 99$ \\
\hline Observers & TH et al & TH et al & TH et al & TH et al & TH et al & TH et al & TH et al & TH et al \\
\hline Wildlife Area & L-Satus & L-Satus & L-Satus & L-Satus & L-Satus & L-Satus & L-Satus & L-Sanus \\
\hline 1991 Cover Type & $\mathbf{A g}$ & $\mathrm{Ag}$ & $r F$ & $\mathrm{rS}$ & PEM & SSG & $\mathbf{R}$ & $\mathbf{r H}$ \\
\hline 1999 Cover Type & $\mathrm{Ag}$ & $\mathbf{A g}$ & $r F$ & rS & PEM & SSG & $\mathbf{R}$ & $\mathbf{r H}$ \\
\hline RAI Cover Type & Ag-pf & Ag-pf & $\mathbf{F}$ & $\mathbf{s}$ & PEM & SSG & $\mathbf{R}$ & $\mathbf{H}$ \\
\hline$\%$ Exotic Shrubs & 1 & 1 & 21 & 7 & 1 & 1 & 1 & 1 \\
\hline \% Exotic grass, herb, or rush & $x$ & $x$ & 3 & 1 & 1 & 1 & 2 & 4 \\
\hline Elapse Time & 4 & 5 & 6 & 6 & 4 & 5 & 4 & 2 \\
\hline Stand Age & $x$ & 10 & 50 & $x$ & $x$ & 10 & $\mathrm{x}$ & $x$ \\
\hline trees, present/absent & 1 & 1 & 2 & 1 & 1 & 1 & 1 & 1 \\
\hline trees, percent cover & 1 & 1 & 7 & 1 & 1 & 1 & 1 & $\mathrm{I}$ \\
\hline trees, average dbh & 1 & 1 & 4 & 1 & 1 & 1 & 1 & 1 \\
\hline trees, max dbh & 1 & 1 & 5 & 1 & 1 & 1 & 1 & 1 \\
\hline trees, height & 1 & 1 & 5 & 1 & 1 & 1 & 1 & 1 \\
\hline basal area & 1 & 1 & 4 & 1 & 1 & 1 & 1 & 1 \\
\hline snags, 4-6"dbh/ac & 1 & 1 & 5 & 1 & 1 & 1 & 1 & 1 \\
\hline snags, 7-9"dbh/ac & 1 & 1 & 5 & 1 & 1 & 1 & 1 & 1 \\
\hline snags, $>10 " \mathrm{dbh} / \mathrm{ac}$ & 1 & 1 & 5 & 1 & 1 & 1 & 1 & 1 \\
\hline shrubs, percent cover & 2 & 3 & 3 & 7 & 1 & 5 & 1 & 1 \\
\hline shrubs, $\%$ deciduous cover & 2 & 1 & 3 & 7 & 1 & 1 & 1 & 1 \\
\hline shrubs, $\%$ hydrophytic cover & 1 & 1 & 3 & 1 & 1 & 1 & 1 & 1 \\
\hline shrubs, average height & 4 & 3 & 4 & 6 & 1 & 4 & 1 & 1 \\
\hline grass-herb, percent cover & 7 & 7 & 5 & 4 & 1 & 4 & 1 & 6 \\
\hline grass, percent cover & 6 & 7 & 5 & 4 & 1 & 4 & 1 & 3 \\
\hline grass as $\%$ of herb \& grass & 4 & 6 & 5 & 3 & 1 & 7 & 1 & 4 \\
\hline 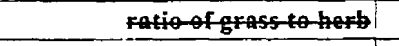 & 1 & 1 & 1 & 1 & 1 & 1 & 1 & 1 \\
\hline herbs, average height & 3 & 3 & 3 & 3 & 1 & 2 & 1 & 3 \\
\hline herbs, percent cover $<2 \mathrm{ft}$ & 5 & 5 & 5 & 3 & 1 & 6 & 1 & 4 \\
\hline SGCM, \% organic cover & 1 & 1 & 1 & 1 & 1 & 1 & 1 & 1 \\
\hline PEM, percent cover & 1 & 1 & 1 & 1 & 7 & 1 & 3 & 1 \\
\hline PAB4, percent cover & 1 & 1 & 1 & 1 & 1 & 1 & 1 & $t$ \\
\hline human disturbance, distance & 4 & 5 & 3 & 5 & 5 & 4 & 3 & 3 \\
\hline quail, distance escape cover & 4 & 6 & 1 & 2 & 1 & 6 & 1 & 2 \\
\hline quail, diameter escape patches & 2 & 4 & 1 & 4 & 1 & 2 & 1 & 2 \\
\hline quall, distance between patches & 5 & 6 & 1 & 2 & 1 & 2 & 1 & 2 \\
\hline goose, nesting babitat & 1 & 1 & 1 & 1 & 1 & 1 & 1 & 1 \\
\hline goose, distance nest/forage & 4 & 4 & 4 & 4 & 4 & 4 & 1 & 3 \\
\hline mallard, dist. nest/rearing & 3 & 2 & 1 & 2 & 2 & 3 & 1 & 3 \\
\hline mallard, height residual cover: & 4 & 4 & 1 & 3 & 1 & 3 & 1 & 4 \\
\hline mallard, percent nest cover & 5 & 5 & 1 & 3 & 1 & 4 & 1 & 6 \\
\hline mallard, ratio PEM to POW & 1 & 1 & 1 & 1 & 4 & 1 & 1 & 1 \\
\hline mallard, water regime & 1 & 1 & 6 & 6 & 3 & 1 & 2 & 5 \\
\hline sandpiper, \% organic cover & 1 & 1 & 1 & 1 & 1 & 1 & 1 & 1 \\
\hline sandpiper, distance nest/water & 1 & 1 & 1 & 1 & 1 & 1 & 1 & 1 \\
\hline mink, $\%$ year with water & 1 & 1 & 5 & 5 & 5 & 1 & 5 & 1 \\
\hline mink, \% tree/shrub cover $330 \mathrm{ft}$ & 1 & 1 & 6 & 6 & 6 & 1 & 4 & 1 \\
\hline mink, \% tree/shrub cover edge & 1 & 1 & 6 & 5 & 5 & 1 & 5 & 1 \\
\hline meadowlark, dist. perches & 3 & 3 & 1 & 1 & 1 & 3 & 1 & 3 \\
\hline heron, dist. nest/foraging & 6 & 7 & 6 & 5 & 5 & 7 & 7 & 7 \\
\hline heron, forage quality & 2 & 2 & 1 & 2 & 4 & 2 & 5 & 1 \\
\hline heron, potential nest sites & 1 & 1 & 1 & 1 & 1 & 1 & 1 & 1 \\
\hline heron, distance potential nests & 6 & 7 & 6 & 5 & 5 & 7 & 7 & 7 \\
\hline
\end{tabular}


Table C-7. Yakama Method Step Values-Attributes, Yakama Nation HEP Analysis.

\begin{tabular}{|c|c|c|c|c|c|c|c|c|}
\hline Line & parameter & 1 & 2 & 3 & 4 & 5 & 6 & 7 \\
\hline 4 & trees, present/absent & absence & presence & & & & & \\
\hline 5 & trees, percent cover & 0 & solitary & $1-5 \%$ & $6-25 \%$ & $26-50 \%$ & $51-75 \%$ & $>76 \%$ \\
\hline 6 & trees, height & 0 & $<10^{\prime}$ & $11-25^{\prime \prime}$ & $26-50^{\prime}$ & $51-100^{\prime}$ & $>100^{\prime}$ & \\
\hline 7 & trees, average dbh & 0 & $<4^{\prime \prime}$ & $4-8 "$ & $8-12^{\prime \prime}$ & $13-24 "$ & $24-36^{\prime \prime}$ & $>36^{\prime \prime}$ \\
\hline 8 & trees, max dbh & 0 & $<4 "$ & $4-8^{\prime \prime}$ & $8-12^{\prime \prime}$ & $13-24 "$ & $24-36 "$ & $>36^{\prime \prime}$ \\
\hline 9 & basal area, sq. meters/hectare & 0 & 5 & 10 & 15 & 20 & 30 & 40 \\
\hline 10 & snags, 4-6"dbh/ac & 0 & 1 & 2 & 3 & 4 & 5 & $>5$ \\
\hline 11 & snags, 7-9"dbh/ac & 0 & 1 & 2 & 3 & 4 & 5 & $>5$ \\
\hline 12 & snags, $>10^{\prime \prime} \mathrm{dbh} / \mathrm{ac}$ & 0 & 1 & 2 & 3 & 4 & 5 & $>5$ \\
\hline 13 & shrubs, percent cover & 0 & solitary & $1-5 \%$ & $6-25 \%$ & $26-50 \%$ & $51-75 \%$ & $>75$ \\
\hline 14 & shrubs, $\%$ deciduous cover & 0 & solitary & $1-5 \%$ & $6-25 \%$ & $26-50 \%$ & $51-75 \%$ & $>75$ \\
\hline 15 & shrubs, $\%$ hydrophytic cover & 0 & solitary & $1-5 \%$ & $6-25 \%$ & $26-50 \%$ & $51-75 \%$ & $>75$ \\
\hline 16 & shrubs, average height & 0 & $<I^{\prime}$ & $1-3^{\prime}$ & $3-6^{\prime}$ & $6-10^{\prime}$ & $>10^{\prime}$ & \\
\hline 17 & herb, percent cover & 0 & solitary & $1-5 \%$ & $6-25 \%$ & $26-50 \%$ & $51-75 \%$ & $>75$ \\
\hline 18 & grass, percent cover & 0 & solitary & $1-5 \%$ & $6-25 \%$ & $26-50 \%$ & $51-75 \%$ & $>75$ \\
\hline 19 & herb \& grass, percent cover & 0 & solitary & $1-5 \%$ & $6-25 \%$ & $26-50 \%$ & $51-75 \%$ & $>75$ \\
\hline 20 & ratio of grass to herb & & & & & & & \\
\hline 21 & herbs, average height & 0 & $<1^{\prime}$ & $1-3^{\prime}$ & $3-6^{\prime}$ & $6-10^{\prime}$ & $>10^{\prime}$ & \\
\hline 22 & herbs, percent cover $<2 \mathrm{ft}$ & 0 & solitary & $1-5 \%$ & $6-25 \%$ & $26-50 \%$ & $51-75 \%$ & $>75$ \\
\hline 23 & SGCM, \% organic cover & 0 & solitary & $1-5 \%$ & $6-25 \%$ & $26-50 \%$ & $51-75 \%$ & $>75$ \\
\hline 24 & PEM, percent cover & 0 & solitary & $1-5 \%$ & $6-25 \%$ & $26-50 \%$ & $51-75 \%$ & $>75$ \\
\hline 25 & PAB4, percent cover & 0 & solitary & $1.5 \%$ & $6-25 \%$ & $26-50 \%$ & $51-75 \%$ & $>75$ \\
\hline 26 & human disturbance, distance & 0 & $<500^{\prime}$ & $<1 / 4$ mile & $1 / 4-1 / 2$ & $>1 / 2$ & $>1$ mile & $>5$ miles \\
\hline 27 & quail, distance to escape cover & 0 & $<100^{\prime}$ & $101-200^{\prime}$ & $201-300^{\prime}$ & $301-500^{\prime}$ & $500-875^{\prime}$ & $>875^{\prime}$ \\
\hline 28 & quail, diameter escape patches & 0 & $<10^{\prime}$ & $11-20^{\prime}$ & $>21^{\prime}$ & & & \\
\hline 29 & quail, distance between patches & 0 & $<30^{\prime}$ & $31-90^{\prime}$ & $91-200^{\prime}$ & $201-300^{\prime}$ & $>300^{\prime}$ & \\
\hline 30 & goose, nesting habitat & 0 & few trees & abundant & & & & \\
\hline 31 & goose, distance nest/forage & 0 & $<1$ mile & $1-2 m$ & $>2$ miles & & & \\
\hline 32 & mallard, distance nest/rearing & 0 & $<1 / 4$ mile & $1 / 4-3 / 4$ & $>3 / 4$ mile & & & \\
\hline 33 & mallard, height residual cover & 0 & $<15^{\prime \prime}$ & $16-24^{\prime \prime}$ & $25-48^{\prime \prime}$ & $>48 "$ & & \\
\hline 34 & mallard, percent nest cover & 0 & solitary & $1-5 \%$ & $6-25 \%$ & $26-50 \%$ & $51-75 \%$ & $>75$ \\
\hline 35 & mallard, ratio PEM to POW & 0 & $<40: 60$ & $40: 60$ & $>40: 60$ & & & \\
\hline 36 & sandpiper, $\%$ organic cover & 0 & solitary & $1-5 \%$ & $6-25 \%$ & $26-50 \%$ & $51-75 \%$ & $>75$ \\
\hline 37 & sandpiper, distance nest/water & 0 & $0-10^{\prime}$ & $11-20^{\prime}$ & $21-50^{\prime}$ & $51-100^{\prime}$ & $101-200^{\prime}$ & $>200^{\prime}$ \\
\hline 38 & mink, \% year with water & 0 & $1-25 \%$ & $26-50 \%$ & $51-75 \%$ & $>75 \%$ & & \\
\hline 39 & mink, $\%$ tree/shrub cover $330 \mathrm{ft}$ & 0 & solitary & $1-5 \%$ & $6-25 \%$ & $26-50 \%$ & $51-75 \%$ & $>75$ \\
\hline 40 & mink, \% tree/shrub cover edge & 0 & solitary & $1-5 \%$ & $6-25 \%$ & $26-50 \%$ & $51-75 \%$ & $>75$ \\
\hline 41 & meadowlark, distance perches & 0 & few & scattered & abundant & & & \\
\hline 42 & heron, distance nest/foraging & 0 & $<500^{\prime}$ & $<1 / 4$ mile & $1 / 4-1 / 2$ & $>1 / 2$ & $>1$ mile & $>2$ miles \\
\hline 43 & heron, forage quality & 0 & $d r y a g-p$ & wet ag-p & hlw mrk & shlw clr & dp mrky & $\mathrm{dp}$ clr \\
\hline 44 & heron, potential nest sites & No & Yes & & & & & \\
\hline 45 & heron, distance potential nests & 0 & $<500^{\prime}$ & $<1 / 4$ mile & $1 / 4-1 / 2$ & $>1 / 2$ & $>1$ mile & $>2$ miles \\
\hline 46 & downy woodpecker & none & & & & & & \\
\hline 47 & black-capped chickadee & none & & & & & & \\
\hline 48 & yellow warbler & none & & & & & & \\
\hline
\end{tabular}

Datasheet Column

few trees 
Table C-7. Yakama Method Step Values-Attributes, Yakama Nation HEP Analysis.

Line

parameter

ariable California Quail

V1 grass and herbs, percent cover

V2 shrubs, average height

V3 escape cover, distance to

V4 escape cover, patch diam/widt

V5 escape cover, distance between

\section{Canada goose}

V1 mature trees present

V3 nest to brood rearing, distance

V4 human disturbance, distance

\section{Mallard}

V3 herb/shrubs cover within $300 \mathrm{ft}$

V4 herb/shrub cover $300-600 \mathrm{ft}$

V5 nesting cover, height

. V6 disturbance, human and dog

V7 ratio PEM/PAB4 to POW

V-8 distance nesting/brood rearing

V-9 water regime

\section{Spotted Sandpiper}

V1 herbs $<2$ ', percent cover

V2 nest to water, distance

V3 organic debris, percent cover

\section{Mink}

V1 surface water, precent of year

V2 tree canopy, percent cover

V3 shrub canopy, percent cover

V4 PEM/PAB4, percent cover

V5 trees, percent cover within 330'

V6 tree/shrub, \% canopy shoreline

\section{Western Meadowlark}

V1 herbs \& grass, percent cover

V2 grasses. percent cover

V3 herb/grass, average height

V4 perch sites, distance

V5 shrubs, percent cover

\section{Black-capped Chickadee}

V1 trees, percent cover

V2 trees, average height

V3 snags, \#/ac 4-10" dbh

\section{Datasheet Column}

12
3

4

5

6

7

$\begin{array}{ccccccc}0 & \text { solitary } & 1-5 \% & 6-25 \% & 26-50 \% & 51-75 \% & >75 \\ 0 & <1^{\prime} & 1-3^{\prime} & 3-6^{\prime} & 6-10^{\prime} & >10^{\prime} & 0 \\ 0 & <100^{\prime} & 101-200^{\prime} & 201-300^{\prime} & 301-500^{\prime} & 500-875^{\prime} & >875^{\prime} \\ 0 & <10^{\prime} & 11-20^{\prime} & >21^{\prime} & 0 & 0 & 0 \\ 0 & <30^{\prime} & 31-90^{\prime} & 91-200^{\prime} & 201-300^{\prime} & >300^{\prime} & 0\end{array}$

$\begin{array}{ccccccc}\text { absence } & \text { presence } & 0 & 0 & 0 & 0 & 0 \\ 0 & <1 \text { mile } & 1-2 \mathrm{~m} & >2 \text { miles } & 0 & 0 & 0 \\ 0 & <500^{\prime} & <1 / 4 \text { mile } & 1 / 4-1 / 2 & >1 / 2 & >1 \text { mile } & >5 \text { miles }\end{array}$

$\begin{array}{rrrccccc}0 & \text { solitary } & 1-5 \% & 6-25 \% & 26-50 \% & 51-75 \% & >75 \\ 0 & <15^{\prime \prime} & 16-24 " & 25-48^{\prime \prime} & >48^{\prime \prime} & 0 & 0 \\ 0 & <500^{\prime} & <1 / 4 \text { mile } & 1 / 4-1 / 2 & >1 / 2 & >1 \text { mile } & >5 \text { miles } \\ 0 & <40: 60 & 40: 60 & >40: 60 & 0 & 0 & 0 \\ 0 & <1 / 4 \text { mile } & 1 / 4-3 / 4 & >3 / 4 \text { mile } & 0 & 0 & 0 \\ 0 & \text { ermanen } & \text { intermit. } & \text { emi-per } & \text { seasonal } & \text { emporar } & \text { intermit } \\ & & \text { exposed } & & & & \text { flooded }\end{array}$

$\begin{array}{ccccccc}0 & \text { solitary } & 1-5 \% & 6-25 \% & 26-50 \% & 51-75 \% & >75 \\ <4 " & 4-8^{\prime \prime} & 8-12 " & 13-24 " & 24-36 " & >36 " & \text { \#REF! } \\ 0 & \text { solitary :; } & 1-5 \% & 6-25 \% & 26-50 \% & 51-75 \% & >75\end{array}$

$\begin{array}{lcccccc}0 & 1-25 \% & 26-50 \% & 51-75 \% & >75 \% & 0 & 0 \\ 0 & \text { solitary } & 1-5 \% & 6-25 \% & 26-50 \% & 51-75 \% & >76 \% \\ 0 & \text { solitary } & 1-5 \% & 6-25 \% & 26-50 \% & 51-75 \% & >75 \\ 0 & \text { solitary } & 1-5 \% & 6-25 \% & 26-50 \% & 51-75 \% & >75 \\ 0 & \text { solitary } & 1-5 \% & 6-25 \% & 26-50 \% & 51-75 \% & >75 \\ 0 & \text { solitary } & 1-5 \% & 6-25 \% & 26-50 \% & 51-75 \% & >75\end{array}$

$\begin{array}{lcccccc}0 & \text { solitary } & 1-5 \% & 6-25 \% & 26-50 \% & 51-75 \% & >75 \\ 0 & \text { solitary } & 1-5 \% & 6-25 \% & 26-50 \% & 51-75 \% & >75 \\ 0 & <1^{\prime} & 1-3^{\prime} & 3-6^{\prime} & 6-10^{\prime} & >10^{\prime} & 0 \\ 0 & \text { few } & \text { scattered } & \text { abundant } & 0 & 0 & 0 \\ 0 & \text { solitary } & 1-5 \% & 6-25 \% & 26-50 \% & 51-75 \% & >75\end{array}$


Table C-7. Yakama Method Step Values-Attributes, Yakama Nation HEP Analysis.

Line

parameter

\section{Yellow Warbler}

V1 decid. shrubs, percent cover

V2 decid. shrubs, average height

V3 hydrophytic shrubs, \% cover

\section{Great Blue Heron}

V1 nesting to feeding, distance

V2 forage quality

V3 human disturbance

V4 nest sites, availability

V5 disturbance at potential nest sit

V6 actual/potential nests, distance

Table 64, continued

Line

parameter

\section{Downy Woodpecker}

- V1 basal area, sq. meters/hectare

V2 snags, 7-9"dbh/ac

\section{Datasheet Column}

12

2

3

4

5

6

7

$\begin{array}{ccccccc}0 & \text { solitary } & 1-5 \% & 6-25 \% & 26-50 \% & 51-75 \% & >75 \\ 0 & <1^{\prime} & 1-3^{\prime} & 3-6^{\prime} & 6-10^{\prime} & >10^{\prime} & 0 \\ 0 & \text { solitary } & 1-5 \% & 6-25 \% & 26-50 \% & 51-75 \% & >75\end{array}$

$\begin{array}{ccccccc}0 & <500^{\prime} & <1 / 4 \text { mile } & 1 / 4-1 / 2 & >1 / 2 & >1 \text { mile } & >2 \text { miles } \\ 0 & \text { dry ag-p } & \text { wet ag-p } & \text { hlw mrk } & \text { shlw clr } & \text { dp mrky } & \text { dp clr } \\ 0 & <500^{\prime} & <1 / 4 \text { mile } & 1 / 4-1 / 2 & >1 / 2 & >1 \text { mile } & >5 \text { miles } \\ \text { No } & \text { Yes } & 0 & 0 & 0 & 0 & 0 \\ 0 & <500^{\prime} & <1 / 4 \text { mile } & 1 / 4-1 / 2 & >1 / 2 & >1 \text { mile } & >5 \text { miles }\end{array}$

Datasheet Column

1

2

3

5

6

7

$\begin{array}{ccccccc}0 & 5 & 10 & 15 & 20 & 30 & 40 \\ 0 & 1 & 2 & 3 & 4 & 5 & >5\end{array}$


Table C-8. Yakama Method Step Values for Species Models, Yakama Nation HEP Analysis.

Datasheet column score (Table C-7) converted to HSI variable values

\begin{tabular}{|c|c|c|}
\hline \multirow[t]{2}{*}{ Variable } & \multirow[t]{2}{*}{ line } & \multirow[b]{2}{*}{ California Quail } \\
\hline & & \\
\hline $\mathrm{VI}$ & 29 & grass and herbs, percent cover \\
\hline V2 & 28 & shrubs, average height \\
\hline V3 & 39 & escape cover, distance to \\
\hline V4 & 40 & escape cover, patch diam/width \\
\hline \multirow[t]{2}{*}{ v5 } & 41 & escape cover, distance between \\
\hline & & Canada goose \\
\hline $\mathrm{V} 1$ & 16 & mature trees present \\
\hline V3 & 42 & nest to brood rearing, distance \\
\hline \multirow[t]{2}{*}{ V4 } & 43 & human disturbance, distance \\
\hline & & Mallard \\
\hline V3 & 46 & herb/shrubs cover with in $300 \mathrm{ft}$ \\
\hline V4 & 46 & herb/shrub cover $300-600 \mathrm{ft}$ \\
\hline$\cdot$ V5 & 45 & nesting cover, height \\
\hline V6 & 38 & disturbance, human and dog \\
\hline v7 & 47 & ratio PEM/PAB4 to POW \\
\hline$V-8$ & 44 & distance nesting/brood rearing \\
\hline & & water reoime \\
\hline
\end{tabular}

\section{datasheet column}

$\begin{array}{ccccccc}1 & 2 & 3 & 4 & 5 & 6 & 7 \\ 0 & 0 & 0.1 & 0.3 & 0.6 & 1 & 0.9 \\ 0 & 0.1 & 0.4 & 0.5 & 0.8 & 1 & \\ 1 & 1 & 0.8 & 0.5 & 0.3 & 0.1 & 0 \\ 0 & 0.3 & 1 & 0.5 & 0 & 0 & 0 \\ 0.3 & 0.4 & 0.75 & 1 & 0.6 & 0.1 & 0\end{array}$

\section{Spotted Sandpiper}

V1 34 herbs $<2$, percent cover

V2 50 nest to water, distance

V3 49 organic debris, percent cover

$\begin{array}{cccccccc}0 & & 0.1 & 0.2 & 0.7 & 1 & 0.5 & 0 \\ 1 & & 1 & 1 & 0.8 & 0.4 & 0 & 0 \\ 1 & \vdots & 1 & 1 & 1 & 1 & 0.7 & 0.3\end{array}$

$\begin{array}{lll} & & \text { Mink } \\ \text { V1 } & 51 & \text { surface water, precent of year } \\ \text { V2 } & 17 & \text { tree canopy, percent cover } \\ \text { V3 } & 25 & \text { shrub canopy, percent cover } \\ \text { V4 } & 36 & \text { PEM/PAB4, percent cover } \\ \text { V5 } & 52 & \text { trees, percent cover within 330' } \\ \text { V6 } & 53 & \text { tree/shrub, \% canopy shoreline }\end{array}$

$\begin{array}{ccccccc}0 & 0 & 0.5 & 0.75 & 1 & & \\ 0.1 & 0.1 & 0.15 & 0.2 & 0.5 & 0.75 & 1 \\ 0.1 & 0.1 & 0.15 & 0.2 & 0.5 & 0.75 & 1 \\ 0 & 0.1 & 0.15 & 0.25 & 0.75 & 1 & 0.9 \\ 0.1 & 0.1 & 0.2 & 0.3 & 0.4 & 0.5 & 1 \\ 0 & 0 & 0.1 & 0.2 & 0.3 & 0.5 & 0.9\end{array}$

Western Meadowlark

V1 29 herbs \& grass, percent cover

V2 $30 \quad$ grasses. percent cover

V3 33 herb/grass, average height

V4 54 perch sites, distance

V5 25 shrubs, percent cover

\section{Black-capped Chickadee}

$\begin{array}{lll}\text { V1 } & 17 & \text { trees, percent cover } \\ \text { V2 } & 20 & \text { trees, average height } \\ \text { V3 } & 23 & \text { snags, \#/ac 4-10" dbh }\end{array}$

$\begin{array}{ccccccc}0 & 0 & 0.05 & 0.1 & 0.3 & 0.7 & 1 \\ 0 & 0 & 0.1 & 0.2 & 0.3 & 0.7 & 1 \\ 0 & 1 & 0.3 & 0 & 0 & 0 & 0 \\ 0 & 0.25 & 0.75 & 1 & 0 & 0 & 0 \\ 1 & 1 & 1 & 0.7 & 0.2 & 0 & 0\end{array}$

$\begin{array}{lcccccc}0 & 0.1 & 0.2 & 0.3 & 0.7 & 1 & 0.7 \\ 0 & 0.1 & 0.2 & 0.7 & 1 & 1 & \\ 0 & 0.5 & 1 & 1 & 1 & 1 & 1\end{array}$


- Table C-8. Yakama Method Step Values for Species Models, Yakama Nation HEP Analysis.

$\begin{array}{ccl}\text { Variable } & \text { line } & \text { Yellow Warbler } \\ \text { V1 } & 26 & \begin{array}{l}\text { decid. shrubs, percent cover } \\ \text { V2 }\end{array} \\ \text { V3 } & 28 & \begin{array}{l}\text { decid. shrubs, average height } \\ \text { hydrophytic shrubs, \% cover }\end{array} \\ & & \\ \text { V1 } & 55 & \text { Great Blue Heron } \\ \text { V2 } & 56 & \text { forage quality } \\ \text { V3 } & 38 & \text { human disturbance } \\ \text { V4 } & 57 & \text { nest sites, availability } \\ \text { V5 } & 58 & \text { disturbance at potential nest sites } \\ \text { V6 } & 58 & \text { actual/potential nests, distance } \\ & & \\ \text { V1 } & 21 & \text { Downy Woodpecker } \\ \text { V2 } & 23 & \text { number of sngs }>6 " \text { per acre }\end{array}$

\begin{tabular}{|c|c|c|c|c|c|c|}
\hline \multicolumn{7}{|c|}{ latasheet column } \\
\hline 1 & 2 & 3 & 4 & 5 & 6 & 7 \\
\hline 0 & 0 & 0.1 & 0.2 & 0.5 & 1 & 0.7 \\
\hline 0 & 0 & 0.25 & 0.5 & 1 & 1 & \\
\hline 0.1 & 0.15 & 0.2 & 0.25 & 0.4 & 0.6 & 0.9 \\
\hline 1 & 1 & 1 & 1 & 1 & 1 & 1 \\
\hline 0.1 & 0.3 & 0.5 & 0.7 & 1 & 0.2 & 0.4 \\
\hline 0 & 0.1 & 0.3 & 0.5 & 1 & 1 & 1 \\
\hline 0 & 1 & 1 & 1 & 1 & 0.7 & 0.5 \\
\hline 0 & 0.5 & 1 & 1 & 1 & 1 & 1 \\
\hline 1 & 1 & 1 & 1 & 1 & 1 & 1 \\
\hline 0 & 0.25 & 0.5 & 1 & 1 & 0.5 & 0 \\
\hline 0 & 0.2 & 0.4 & 0.8 & 1 & 1 & 1 \\
\hline
\end{tabular}


Table C-9. Replace Column Number from Field Sheet With HSI Score.

\begin{tabular}{|c|c|c|c|c|c|c|c|c|c|}
\hline Serial Number & 55 & 69 & 80 & 28 & 41 & 44 & 45 & 49 & 4 \\
\hline RAI Cover Type & $\mathrm{Ag}-\mathrm{c}$ & Ag-cf & Ag-cf & Ag*p & Ag-p & Ag-p & Ag-p & Ag-p & Ag-pf \\
\hline California Quail & & & & & & & & & 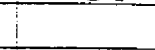 \\
\hline V1 & 0.90 & 0.60 & 1.00 & 0.90 & 0.90 & 1.00 & 1.00 & 0.90 & 1.00 \\
\hline V2 & 0.00 & 0.00 & 0.00 & 0.00 & 0.00 & 0.40 & 0.40 & 0.00 & 0.00 \\
\hline V3 & 0.10 & 0.30 & 0.30 & 1.00 & 0.80 & 0.30 & 0.30 & 1.00 & 1.00 \\
\hline V4 & 0.00 & 0.50 & 0.30 & 0.50 & 1.00 & 1.00 & 1.00 & 1.00 & 1.00 \\
\hline V5 & 0.30 & 0.40 & 0.10 & 0.30 & 1.00 & 0.10 & 0.10 & 0.75 & 0.30 \\
\hline HSI & & 0.99 & 1.00 & 1.00 & 1.00 & 1.00 & 1.00 & 1.00 & 1.00 \\
\hline Plots $>1.0$ & & & $\mathrm{XX}$ & $\mathrm{XX}$ & $\mathrm{XX}$ & $\mathrm{XX}$ & $\mathrm{XX}$ & $\mathrm{XX}$ & $\mathrm{XX}$ \\
\hline & & & & & & & & & \\
\hline \multicolumn{10}{|l|}{ Canada Goose } \\
\hline $\mathrm{V} 1$ & 0.00 & 0.00 & 0.00 & 0.00 & 0.00 & 0.00 & 0.00 & 0.00 & 0.00 \\
\hline V3 & 1.00 & 1.00 & 1.00 & 1.00 & 1.00 & 1.00 & 1.00 & 1.00 & 1.00 \\
\hline V4 & 0.00 & 0.10 & 1.00 & 0.00 & 0.10 & 0.00 & 0.00 & 0.00 & 0.00 \\
\hline \multirow[t]{2}{*}{ HSI } & & & & 0.71 & 0.74 & 0.71 & 0.71 & 0.71 & $0.7 \overline{1}$ \\
\hline & & & & 1 & & & & & \\
\hline \multicolumn{10}{|l|}{ Mallard } \\
\hline$\sqrt{ } 3$ & 0.00 & 0.00 & 0.00 & 0.50 & 0.10 & 0.05 & 0.05 & 0.00 & 0.10 \\
\hline$\sqrt{4}$ & 0.00 & 0.00 & 0.00 & 0.50 & 0.10 & 0.05 & 0.05 & 0.00 & 0.10 \\
\hline V5 & 1.00 & 0.30 & 1.00 & 0.40 & 1.00 & 0.30 & 0.30 & 0.30 & 0.30 \\
\hline V6 & 1.00 & 1.00 & 1.00 & 1.00 & 1.00 & 1.00 & 1.00 & 1.00 & 0.50 \\
\hline V7 & 0.00 & 0.00 & 0.00 & 0.00 & 0.00 & 0.00 & 0.00 & 0.00 & 0.00 \\
\hline V-8 & 1.00 & 1.00 & 0.50 & 1.00 & 1.00 & 1.00 & 1.00 & 1.00 & 1.00 \\
\hline V-9 & 0.00 & 0.00 & 0.00 & 0.00 & 0.00 & 0.00 & 0.00 & 0.00 & 0.00 \\
\hline \multirow[t]{2}{*}{ HSI } & 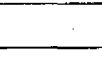 & & 0.00 & 0.45 & 0.32 & 0.12 & 0.12 & 0.00 & 0.09 \\
\hline & & & & & 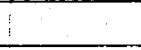 & & & & \\
\hline Spotted Sandpiper & & & & & 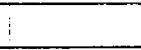 & & $\cdots$ & & \\
\hline V1 & 0.00 & 1.00 & 0.00 & 0.70 & 0.00 & 0.00 & 0.00 & 0.00 & 0.20 \\
\hline V2 & 1.00 & 1.00 & 1.00 & 1.00 & 1.00 & 1.00 & 1.00 & 1.00 & 1.00 \\
\hline V3 & 1.00 & 1.00 & 1.00 & 1.00 & 1.00 & 1.00 & 1.00 & 1.00 & 1.00 \\
\hline \multirow[t]{2}{*}{ HSI } & & & & & $!$ & & & & \\
\hline & & & & & $i$ & & & & \\
\hline Mink & & & & 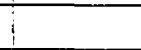 & 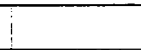 & & & & \\
\hline$v_{1}$ & 0.00 & 0.00 & 0.00 & 0.00 & 0.00 & 0.00 & 0.00 & 0.00 & 0.00 \\
\hline V2 & 0.10 & 0.10 & 0.10 & 0.10 & 0.10 & 0.10 & 0.10 & 0.10 & 0.10 \\
\hline V3 & 0.10 & 0.10 & 0.10 & 0.10 & 0.10 & 0.20 & 0.20 & 0.10 & 0.10 \\
\hline $\mathrm{V4}$ & 0.00 & 0.00 & 0.00 & 0.00 & 0.00 & 0.00 & 0.00 & 0.00 & 0.00 \\
\hline V5 & 0.10 & 0.10 & 0.10 & 0.10 & 0.10 & 0.10 & 0.10 & 0.10 & 0.10 \\
\hline V6 & 0.00 & 0.00 & 0.00 & 0.00 & 0.00 & 0.00 & 0.00 & 0.00 & 0.00 \\
\hline$\overline{\mathrm{HSI}}$ & & & & & & & & & \\
\hline
\end{tabular}


Table C-9. Replace Column Number from Field Sheet With HSI Score.

\begin{tabular}{|c|c|c|c|c|c|c|c|c|c|}
\hline Serial Number & 55 & 69 & 80 & 28 & 41 & 44 & 45 & 49 & 4 \\
\hline RAI Cover Type & Ag-c & Ag-cf & Ag-cf & Ag-p & Ag-p & Ag-p & Ag-p & Ag-p & Ag-pf \\
\hline \multicolumn{10}{|l|}{ Meadowlark } \\
\hline V1 & 1.00 & 0.30 & 0.70 & 1.00 & 1.00 & 0.70 & 0.70 & 1.00 & 0.70 \\
\hline V2 & 1.00 & 0.30 & 0.70 & 1.00 & 1.00 & 0.70 & 0.70 & 1.00 & 0.70 \\
\hline V3 & 0.00 & 0.30 & 1.00 & 0.30 & 1.00 & 1.00 & 1.00 & 1.00 & 1.00 \\
\hline$\overline{\mathrm{V} 4}$ & 0.75 & 0.25 & 0.75 & 1.00 & 0.75 & 0.25 & 0.25 & 0.25 & 0.25 \\
\hline V5 & 1.00 & 1.00 & 1.00 & 1.00 & 1.00 & 0.70 & 0.70 & 1.00 & 1.00 \\
\hline \multirow[t]{2}{*}{ HSI } & & & & 0.55 & 0.87 & 0.25 & 0.25 & 0.50 & 0.35 \\
\hline & & & & & & & & & \\
\hline \multicolumn{10}{|l|}{ Chickadee } \\
\hline V1 & & & & & & & & & \\
\hline \multicolumn{10}{|l|}{$\mathrm{V} 2$} \\
\hline \multicolumn{10}{|l|}{ V3 } \\
\hline \multicolumn{10}{|l|}{ HSI } \\
\hline & & & & & & & & & \\
\hline \multicolumn{10}{|l|}{ Yellow Warbler } \\
\hline $\mathrm{V} 1$ & & & & & & & & & \\
\hline v2 & & & & & & & & & \\
\hline $\mathrm{v} 3$ & & & & & & & & & \\
\hline \multirow[t]{2}{*}{ HSI } & & & & & & & & & \\
\hline & & & & & & & & & \\
\hline Great Blue Heron & & & & & & & & & \\
\hline V1 & 1.00 & 1.00 & 1.00 & 1.00 & 1.00 & 1.00 & 1.00 & 1.00 & 1.00 \\
\hline V2 & 0.10 & 0.30 & 0.30 & 0.50 & 0.30 & 0.30 & 0.30 & 0.30 & 0.10 \\
\hline$\sqrt{3}$ & 1.00 & 1.00 & 0.50 & 1.00 & 1.00 & 1.00 & 1.00 & 1.00 & 0.30 \\
\hline $\mathrm{V} 4$ & 0.00 & 0.00 & 0.00 & 0.00 & 0.00 & 0.00 & 0.00 & 0.00 & 0.00 \\
\hline V5 & 1.00 & 0.50 & 1.00 & 1.00 & 1.00 & 1.00 & 1.00 & 1.00 & 1.00 \\
\hline V6 & 1.00 & 1.00 & 1.00 & 1.00 & 1.00 & 1.00 & 1.00 & 1.00 & 1.00 \\
\hline \multirow[t]{2}{*}{ HSI } & & & & 0.50 & 0.30 & 0.30 & 0.30 & 0.30 & 0.03 \\
\hline & & & & & & & ( & & ( \\
\hline \multicolumn{2}{|l|}{ Downy Woodpecker } & & & & & & & & \\
\hline \multicolumn{10}{|l|}{ V1 } \\
\hline \multicolumn{10}{|l|}{ V2 } \\
\hline \multirow[t]{3}{*}{$\mathrm{HSI}$} & & & & & & & 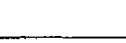 & & \\
\hline & & & & & & & 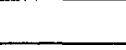 & & \\
\hline & & & & & & & & & \\
\hline
\end{tabular}


Table C-9. Replace Column Number from Field Sheet With HSI Score.

\begin{tabular}{|c|c|c|c|c|c|c|c|c|c|}
\hline Serial Number & 5 & 7 & 15 & 17 & 20 & 32 & 46 & 52 & 54 \\
\hline RAI Cover Type & Ag-pf & Ag-pf & Ag-pf & Ag-pf & Ag-pf & Ag-pf & Ag-pf & Ag-pf & Ag-p \\
\hline \multicolumn{10}{|l|}{ California Quail } \\
\hline V1 & 1.00 & 0.90 & 0.90 & 0.90 & 0.90 & 0.90 & 0.90 & 1.00 & 0.90 \\
\hline V2 & 0.00 & 0.00 & 0.00 & 0.00 & 1.00 & 0.40 & 0.40 & 0.80 & 0.50 \\
\hline $\mathrm{V} 3$ & 1.00 & 1.00 & 0.80 & 1.00 & 1.00 & 0.30 & 1.00 & 1.00 & 0.50 \\
\hline $\mathrm{V4}$ & 0.50 & 0.50 & 0.30 & 0.00 & 0.50 & 0.50 & 1.00 & 1.00 & 0.30 \\
\hline V5 & 0.40 & 0.40 & 1.00 & 0.30 & 1.00 & 0.60 & 1.00 & 1.00 & $0.7 \overline{5}$ \\
\hline HSI & 1.00 & 1.00 & 1.00 & 0.90 & 1.00 & 1.00 & 1.00 & 1.00 & 1.00 \\
\hline \multirow[t]{2}{*}{ Plots $>1.0$} & $\mathrm{XX}$ & $\mathrm{XX}$ & $\mathrm{XX}$ & & $\mathrm{XX}$ & $\mathrm{XX}$ & $\mathrm{XX}$ & $\mathrm{XX}$ & $\mathrm{XX}$ \\
\hline & & & & & & & $i$ & & \\
\hline \multicolumn{10}{|l|}{ Canada Goose } \\
\hline V1 & 0.00 & 0.00 & 0.00 & 0.00 & 0.00 & 0.00 & 0.00 & 0.00 & 0.00 \\
\hline V3 & 1.00 & 1.00 & 1.00 & 1.00 & 1.00 & 1.00 & 1.00 & 1.00 & 1.00 \\
\hline V 4 & 0.10 & 1.00 & 0.10 & 0.10 & 0.10 & 0.00 & 0.00 & 0.10 & 0.10 \\
\hline \multirow[t]{2}{*}{ HSI } & 0.74 & 1.00 & 0.74 & 0.74 & 0.74 & 0.71 & 0.71 & 0.74 & 0.74 \\
\hline & & & & & & & 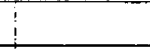 & & \\
\hline \multicolumn{10}{|l|}{ Mallard } \\
\hline V3 & 0.00 & 0.10 & 0.20 & 1.00 & 1.00 & 0.20 & 0.20 & 1.00 & 0.20 \\
\hline$\sqrt{4}$ & 0.00 & 0.10 & 0.20 & 1.00 & 1.00 & 0.20 & 0.20 & 1.00 & 0.20 \\
\hline V5 & 0.30 & 0.30 & 1.00 & 0.30 & 1.00 & 1.00 & 1.00 & 0.30 & 1.00 \\
\hline V6 & 0.50 & 1.00 & 0.50 & 1.00 & 1.00 & 1.00 & 1.00 & 1.00 & 1.00 \\
\hline V7 & 0.00 & 0.00 & 0.00 & 0.00 & 0.00 & 0.00 & 0.00 & 0.00 & 0.00 \\
\hline V-8 & 1.00 & 1.00 & 0.50 & 1.00 & 0.50 & 1.00 & 1.00 & 1.00 & 1.00 \\
\hline V-9 & 0.00 & 0.00 & 0.00 & 0.00 & 0.00 & 0.00 & 0.00 & 0.00 & 0.00 \\
\hline \multirow[t]{2}{*}{ HSI } & 0.00 & 0.17 & 0.22 & 0.55 & 1.00 & 0.45 & 0.45 & 0.55 & 0.45 \\
\hline & & & & & & & & & \\
\hline \multicolumn{2}{|l|}{ Spotted Sandpiper } & & & & & & $\cdots$ & & \\
\hline V1 & 0.00 & 0.50 & 0.50 & 0.70 & 0.70 & 1.00 & 0.00 & 0.70 & 0.00 \\
\hline $\mathbf{V 2}$ & 1.00 & 1.00 & 1.00 & 1.00 & 1.00 & 1.00 & 1.00 & 1.00 & 1.00 \\
\hline V3 & 1.00 & 1.00 & 1.00 & 1.00 & 1.00 & 1.00 & 1.00 & 1.00 & 1.00 \\
\hline \multicolumn{10}{|l|}{ HSI } \\
\hline & & & & & & & & & \\
\hline \multicolumn{10}{|l|}{ Mink } \\
\hline $\mathrm{V} 1$ & 0.00 & 0.00 & 0.00 & 0.00 & 0.00 & 0.00 & 0.00 & 0.00 & 0.00 \\
\hline V2 & 0.10 & 0.10 & 0.10 & 0.10 & 0.10 & 0.10 & 0.10 & 0.10 & 0.10 \\
\hline $\mathrm{V} 3$ & 0.10 & 0.10 & 0.10 & 0.10 & 0.20 & 0.15 & 0.20 & 0.20 & 0.20 \\
\hline V4 & 0.00 & 0.00 & 0.00 & 0.00 & 0.00 & 0.00 & 0.00 & 0.00 & 0.00 \\
\hline V5 & 0.10 & 0.10 & 0.10 & 0.10 & 0.10 & 0.10 & 0.10 & 0.10 & 0.10 \\
\hline V6 & 0.00 & 0.00 & 0.00 & 0.00 & 0.00 & 0.00 & 0.00 & 0.00 & 0.00 \\
\hline HSI & & & & & & & & & \\
\hline
\end{tabular}


Table C-9. Replace Column Number from Field Sheet With HSI Score.

\begin{tabular}{|c|c|c|c|c|c|c|c|c|c|}
\hline Serial Number & 5 & 7 & 15 & 17 & 20 & 32 & 46 & 52 & 54 \\
\hline RAI Cover Type & Ag-pf & Ag-pf & Ag-pf & Ag-pf & Ag-pf & Ag-pf & Ag-pf & Ag-pf & Ag-pf \\
\hline \multicolumn{10}{|l|}{ Meadowlark } \\
\hline $\mathrm{V1}$ & 0.70 & 1.00 & 1.00 & 1.00 & 1.00 & 1.00 & 1.00 & $0 . \overline{70}$ & 1.00 \\
\hline V2 & 0.70 & 1.00 & 0.30 & 0.30 & 1.00 & 1.00 & 1.00 & 0.70 & 1.00 \\
\hline V3 & 1.00 & 1.00 & 0.30 & 0.30 & 0.30 & 0.30 & 0.30 & 0.30 & 0.30 \\
\hline V4 & 0.25 & 0.25 & 0.75 & 0.25 & 0.75 & 0.75 & 0.75 & 0.25 & 0.75 \\
\hline V5 & 1.00 & 1.00 & 1.00 & 1.00 & 0.70 & 1.00 & 0.70 & 0.70 & 0.70 \\
\hline HSI & 0.35 & 0.50 & 0.26 & 0.15 & 0.33 & 0.47 & 0.33 & 0.13 & 0.33 \\
\hline & & & & & & & & 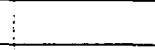 & \\
\hline \multicolumn{10}{|l|}{ Chickadee } \\
\hline \multicolumn{10}{|l|}{$\mathrm{V1}$} \\
\hline \multicolumn{10}{|l|}{ V2 } \\
\hline V3 & & & & & & & & & \\
\hline \multicolumn{10}{|l|}{ HSI } \\
\hline & & & & & & & & & \\
\hline \multicolumn{10}{|l|}{ Yellow Warbler } \\
\hline V1 & & & & & & & & & \\
\hline \multicolumn{10}{|l|}{$\sqrt{2}$} \\
\hline \multicolumn{10}{|l|}{ V3 } \\
\hline \multirow{2}{*}{\multicolumn{10}{|c|}{ HSI }} \\
\hline & & & & & & & & & \\
\hline \multicolumn{10}{|l|}{ Great Blue Heron } \\
\hline V1 & 1.00 & 1.00 & 1.00 & 1.00 & 1.00 & 1.00 & 1.00 & 1.00 & 1.00 \\
\hline V2 & 0.30 & 0.30 & 0.30 & 0.30 & 0.30 & 0.30 & 0.30 & 0.30 & 0.30 \\
\hline$\sqrt{3}$ & 0.30 & 0.50 & 0.30 & 1.00 & 1.00 & 1.00 & 1.00 & 0.50 & 1.00 \\
\hline V4 & 0.00 & 0.00 & 0.00 & 0.00 & 0.00 & 0.00 & 0.00 & 0.00 & 0.00 \\
\hline V5 & 0.00 & 1.00 & 1.00 & 1.00 & 1.00 & 1.00 & 1.00 & 1.00 & 1.00 \\
\hline V6 & 1.00 & 1.00 & 1.00 & 1.00 & 1.00 & 1.00 & 1.00 & 1.00 & 1.00 \\
\hline \multirow[t]{2}{*}{ HSI } & 0.09 & 0.15 & 0.09 & 0.30 & 0.30 & 0.30 & 0.30 & 0.15 & 0.30 \\
\hline & & & & & & & & & \\
\hline \multicolumn{10}{|l|}{ Downy Woodpecke } \\
\hline \multicolumn{10}{|l|}{ V1 } \\
\hline \multicolumn{10}{|l|}{ V2 } \\
\hline \multicolumn{10}{|l|}{ HSI } \\
\hline 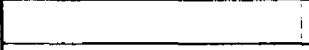 & & & & & & & & $\vdots$ & \\
\hline 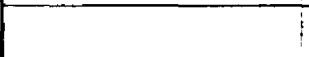 & & & & & & & & 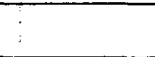 & \\
\hline
\end{tabular}


Table C-9. Replace Column Number from Field Sheet With HSI Score.

\begin{tabular}{|c|c|c|c|c|c|c|c|c|c|}
\hline Serial Number & 58 & 81 & 82 & 10 & 22 & 23 & 24 & 25 & 34 \\
\hline RAI Cover Type & Ag-pf & Ag-pf & Ag-pf & $\mathbf{F}$ & $\mathbf{F}$ & $\mathbf{F}$ & $\mathbf{F}$ & $\mathbf{F}$ & $F$ \\
\hline \multicolumn{10}{|l|}{ California Quail } \\
\hline V1 & 0.90 & 0.90 & 0.90 & 0.90 & 1.00 & 0.60 & 0.90 & 0.30 & 1.00 \\
\hline V2 & 0.00 & 0.50 & 0.40 & 0.80 & 0.80 & 1.00 & 0.80 & 0.80 & 0.50 \\
\hline V3 & 0.30 & 0.50 & 0.10 & 1.00 & 1.00 & 1.00 & 1.00 & 1.00 & 1.00 \\
\hline V4 & 1.00 & 0.30 & 0.50 & 0.00 & 0.00 & 0.00 & 0.00 & 0.00 & 0.00 \\
\hline V5 & 1.00 & 0.60 & 0.10 & 0.30 & 0.30 & 0.30 & 0.30 & 0.30 & $0 . \overline{30}$ \\
\hline HSI & 1.00 & 1.00 & 1.00 & & & & & & \\
\hline \multirow[t]{2}{*}{ Plots $>1.0$} & $\mathrm{XX}$ & $\mathrm{XX}$ & $\mathrm{XX}$ & & & & & & \\
\hline & & & & & & & & 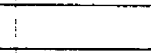 & \\
\hline \multicolumn{10}{|l|}{ Canada Goose } \\
\hline V1 & 0.00 & 0.00 & 0.00 & 1.00 & 1.00 & 1.00 & 1.00 & 1.00 & 1.00 \\
\hline V3 & 1.00 & 1.00 & 1.00 & 1.00 & 1.00 & 1.00 & 1.00 & 1.00 & 0.50 \\
\hline V4 & 0.10 & 1.00 & 1.00 & 0.00 & 1.00 & 0.00 & 0.00 & 0.00 & 0.10 \\
\hline \multirow[t]{2}{*}{ HSI } & 0.74 & 1.00 & 1.00 & 0.71 & 1.00 & 0.71 & 0.71 & 0.71 & 0.55 \\
\hline & & & & & & & & $:$ & \\
\hline Mallard & & & & & & & & 1 & \\
\hline V3 & 0.50 & 0.50 & 0.50 & 0.00 & 0.00 & 0.50 & 0.00 & 0.00 & 0.00 \\
\hline $\mathrm{V} 4$ & 0.50 & 0.50 & 0.50 & 0.00 & 0.00 & 0.50 & 0.00 & 0.00 & 0.00 \\
\hline V5 & 0.30 & 1.00 & 1.00 & 0.00 & 0.00 & 0.00 & 0.00 & 0.00 & 0.00 \\
\hline $\mathrm{V} 6$ & 1.00 & 1.00 & 1.00 & 1.00 & 1.00 & 1.00 & 1.00 & 1.00 & 1.00 \\
\hline V7 & 0.00 & 0.00 & 0.00 & 0.00 & 0.00 & 0.00 & 0.00 & 0.00 & 0.00 \\
\hline V-8 & 1.00 & 0.50 & 1.00 & 1.00 & 1.00 & 1.00 & 1.00 & 1.00 & 1.00 \\
\hline V-9 & 0.00 & 0.00 & 0.00 & 0.00 & 0.00 & 0.00 & 0.00 & 0.00 & 0.00 \\
\hline \multirow[t]{2}{*}{ HSI } & 0.39 & 0.71 & 0.71 & & & & & & \\
\hline & & & & & & & & $:$ & \\
\hline \multicolumn{2}{|l|}{ Spotted Sandpiper } & & & & & & $\cdots$ & & \\
\hline V1 & 0.00 & 1.00 & 1.00 & 0.10 & 0.20 & 0.70 & 1.00 & 0.70 & 0.20 \\
\hline $\mathbf{V 2}$ & 1.00 & 1.00 & 1.00 & 1.00 & 1.00 & 1.00 & 1.00 & 1.00 & 1.00 \\
\hline V3 & 1.00 & 1.00 & 1.00 & 1.00 & 1.00 & 1.00 & 1.00 & 1.00 & 1.00 \\
\hline \multirow[t]{2}{*}{$\mathrm{HSI}$} & & & & 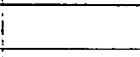 & & & & & \\
\hline & & & & $i$ & & & & & \\
\hline Mink & & & & i & & & & & \\
\hline V1 & 0.00 & 0.00 & 0.00 & 1.00 & 0.00 & 0.00 & 1.00 & 1.00 & 0.50 \\
\hline V2 & 0.10 & 0.10 & 0.10 & 0.20 & 0.75 & 0.50 & 0.75 & 0.75 & 0.50 \\
\hline v3 & 0.10 & 0.10 & 0.15 & 0.20 & 0.20 & 0.75 & 0.50 & 1.00 & 0.50 \\
\hline V4 & 0.00 & 0.00 & 0.00 & 0.00 & 0.00 & 0.00 & 0.00 & 0.00 & 0.00 \\
\hline V5 & 0.10 & 0.10 & 0.10 & 0.40 & 0.50 & 0.50 & 1.00 & 1.00 & 0.50 \\
\hline V6 & 0.00 & 0.00 & 0.00 & 0.30 & 0.70 & 0.70 & 1.00 & 1.00 & 0.70 \\
\hline \multicolumn{2}{|l|}{ HSI } & & & 0.40 & 0.73 & 0.88 & 1.00 & 1.00 & 0.75 \\
\hline
\end{tabular}


. Table C-9. Replace Column Number from Field Sheet With HSI Score.

\begin{tabular}{|c|c|c|c|c|c|c|c|c|c|}
\hline Serial Number & 58 & 81 & 82 & 10 & 22 & 23 & 24 & 25 & 34 \\
\hline RAI Cover Type & Ag-pf & Ag-pf & Ag-pf & $\mathbf{F}$ & $\mathbf{F}$ & $\mathbf{F}$ & $\mathbf{F}$ & $\mathbf{F}$ & $\mathbf{F}$ \\
\hline & & L. & & & & 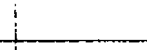 & & & \\
\hline \multicolumn{10}{|l|}{ Meadowlark } \\
\hline V1 & 1.00 & 1.00 & 1.00 & 1.00 & 0.70 & 0.30 & 1.00 & 0.10 & 0.70 \\
\hline V2 & 1.00 & 0.70 & 1.00 & 1.00 & 0.70 & 0.20 & 1.00 & 0.20 & 0.70 \\
\hline V3 & 0.30 & 0.30 & 0.30 & 0.00 & 0.00 & 1.00 & 0.00 & 0.00 & 1.00 \\
\hline V4 & 0.25 & 0.75 & 0.75 & 0.00 & 0.00 & 0.00 & 0.00 & 0.00 & 0.00 \\
\hline V5 & 1.00 & 1.00 & 1.00 & 0.70 & 0.70 & 0.00 & 0.20 & 0.00 & 0.20 \\
\hline \multirow[t]{2}{*}{ HSI } & 0.27 & 0.40 & 0.47 & & & $\vdots$ & & & \\
\hline & & L & & & & $\vdots$ & & : & \\
\hline Chickadee & & & & & & $\vdots$ & & 1 & \\
\hline V1 & & & & 0.30 & 1.00 & 0.70 & 1.00 & 1.00 & 0.70 \\
\hline V2 & & & & 0.70 & 1.00 & 1.00 & 1.00 & 1.00 & 1.00 \\
\hline V3 & & & & 1.00 & 1.00 & 0.00 & 1.00 & 1.00 & 1.00 \\
\hline \multirow[t]{2}{*}{ HSI } & & & & 0.59 & 1.00 & 0.00 & 1.00 & 1.00 & 0.89 \\
\hline & & & & & & i & & & \\
\hline \multicolumn{10}{|l|}{ Yellow Warbler } \\
\hline \multicolumn{10}{|l|}{ V1 } \\
\hline \multicolumn{10}{|l|}{ V2 } \\
\hline \multicolumn{10}{|l|}{ v3 } \\
\hline \multirow[t]{2}{*}{ HSI } & & & & & & & & & \\
\hline & & & & & & & & & \\
\hline \multicolumn{10}{|l|}{ Great Blue Heron } \\
\hline V1 & 1.00 & 1.00 & 1.00 & 1.00 & 1.00 & 1.00 & 1.00 & 1.00 & 1.00 \\
\hline V2 & 0.30 & 0.30 & 0.30 & 1.00 & 0.50 & 0.30 & 0.10 & 0.10 & 0.10 \\
\hline$\overline{3}$ & 1.00 & 0.50 & 1.00 & 1.00 & 1.00 & 1.00 & 1.00 & 1.00 & 1.00 \\
\hline V4 & 0.00 & 0.00 & 0.00 & 1.00 & 1.00 & 1.00 & 1.00 & 1.00 & 1.00 \\
\hline V5 & 1.00 & 1.00 & 1.00 & 0.50 & 0.00 & 1.00 & $0.00^{\circ}$ & 0.00 & 1.00 \\
\hline V6 & 1.00 & 1.00 & 1.00 & 1.00 & 1.00 & 1.00 & 1.00 & 1.00 & 1.00 \\
\hline HSI & 0.30 & 0.15 & 0.30 & 0.71 & 0.00 & 0.55 & 0.00 & 0.00 & 0.32 \\
\hline Downy Woodpecke & & & & & & $i$ & & & \\
\hline V1 & & & & 1.00 & 1.00 & 1.00 & 1.00 & 1.00 & 1.00 \\
\hline $\mathbf{V} 2$ & & & & 0.80 & 0.80 & 0.00 & 0.80 & 0.80 & 1.00 \\
\hline \multirow[t]{3}{*}{ HSI } & & & & 0.80 & 0.80 & 0.00 & 0.80 & 0.80 & 1.00 \\
\hline & & & & & & & & $\vdots$ & \\
\hline & & & & & & & & & \\
\hline
\end{tabular}


Table C-9. Replace Column Number from Field Sheet With HSI Score.

\begin{tabular}{|c|c|c|c|c|c|c|c|c|c|}
\hline Serial Number & 71 & 73 & 83 & 1 & 3 & 14 & 39 & 65 & 67 \\
\hline RAI Cover Type & $\mathbf{F}$ & $\mathbf{F}$ & $\mathbf{F}$ & $\mathbf{G}$ & $\mathbf{G}$ & $\mathbf{G}$ & $\mathbf{G}$ & $\mathbf{G}$ & G \\
\hline \multicolumn{10}{|l|}{ California Quail } \\
\hline V1 & 0.10 & 0.60 & 0.60 & 1.00 & 0.00 & 0.90 & 0.90 & 0.90 & 1.00 \\
\hline V2 & 0.80 & 0.50 & 0.50 & 0.00 & 0.00 & 0.00 & 0.50 & 0.00 & 0.00 \\
\hline V3 & 1.00 & 1.00 & 1.00 & 1.00 & 1.00 & 0.80 & 0.30 & 0.50 & 0.50 \\
\hline V4 & 0.00 & 0.00 & 0.00 & 1.00 & 1.00 & 0.50 & 0.30 & 1.00 & 1.00 \\
\hline V5 & 0.30 & 0.30 & 0.30 & 0.30 & 0.30 & 1.00 & 0.10 & 0.40 & 1.00 \\
\hline HSI & & & & 1.00 & 0.67 & 1.00 & 1.00 & 1.00 & 1.00 \\
\hline \multirow[t]{2}{*}{ Plots > 1.0 } & & & & $\mathrm{XX}$ & & $\mathrm{XX}$ & $\mathrm{XX}$ & $\mathrm{XX}$ & $\mathrm{x} x$ \\
\hline & & & & & & & & & \\
\hline \multicolumn{10}{|l|}{ Canada Goose } \\
\hline V1 & 1.00 & 1.00 & 1.00 & 0.00 & 0.00 & 0.00 & 0.00 & 0.00 & 0.00 \\
\hline V3 & 1.00 & 1.00 & 1.00 & 1.00 & 1.00 & 1.00 & 1.00 & 1.00 & 1.00 \\
\hline V4 & 0.00 & 0.10 & 1.00 & 0.50 & 0.50 & 0.50 & 0.10 & 0.10 & 0.10 \\
\hline \multirow[t]{2}{*}{ HSI } & 0.71 & 0.74 & 1.00 & 0.87 & 0.87 & 0.87 & 0.74 & 0.74 & 0.74 \\
\hline & & & & & & & & 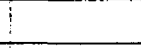 & \\
\hline \multicolumn{10}{|l|}{ Mallard } \\
\hline v3 & 0.00 & 0.00 & 0.00 & 0.80 & 0.10 & 0.10 & 0.10 & 0.80 & 0.20 \\
\hline $\mathrm{V} 4$ & 0.00 & 0.00 & 0.00 & 0.80 & 0.10 & 0.10 & 0.10 & 0.80 & 0.20 \\
\hline V5 & 0.00 & 0.00 & 0.00 & 0.00 & 0.30 & 1.00 & 0.30 & 0.30 & 1.00 \\
\hline v6 & 1.00 & 1.00 & 0.50 & 0.50 & 0.50 & 1.00 & 1.00 & 1.00 & 1.00 \\
\hline V7 & 0.00 & 0.00 & 0.00 & 0.00 & 0.00 & 0.00 & 0.00 & 0.00 & 0.00 \\
\hline$V-8$ & 1.00 & 1.00 & 1.00 & 1.00 & 1.00 & 1.00 & 1.00 & 1.00 & 1.00 \\
\hline V-9 & 0.00 & 0.00 & 0.00 & 0.00 & 0.00 & 0.00 & 0.00 & 0.00 & 0.00 \\
\hline \multirow[t]{2}{*}{ HSI } & & & & 0.00 & 0.09 & 0.32 & 0.17 & 0.49 & 0.45 \\
\hline & & & & & & & & 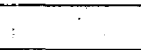 & \\
\hline \multicolumn{2}{|l|}{ Spotted Sandpiper } & & & & & & $\cdots$ & & \\
\hline $\mathrm{V1}$ & 0.50 & 0.20 & 1.00 & 1.00 & 0.50 & 0.00 & 0.50 & 0.20 & 0.00 \\
\hline V2 & 1.00 & 1.00 & 1.00 & 1.00 & 1.00 & 1.00 & 1.00 & 1.00 & 1.00 \\
\hline V3 & 1.00 & 1.00 & 1.00 & 1.00 & 1.00 & 1.00 & 1.00 & 1.00 & 1.00 \\
\hline \multicolumn{10}{|l|}{ HSI } \\
\hline & & & & & & & & & \\
\hline \multicolumn{10}{|l|}{ Mink } \\
\hline V1 & 0.50 & 0.50 & 1.00 & 0.00 & 0.00 & 0.00 & 0.00 & 0.00 & 0.00 \\
\hline V 2 & 1.00 & 0.75 & 1.00 & 0.10 & 0.10 & 0.10 & 0.10 & 0.10 & 0.10 \\
\hline v3 & 0.20 & 1.00 & 0.15 & 0.10 & 0.10 & 0.10 & 0.10 & 0.10 & 0.10 \\
\hline V4 & 0.00 & 0.00 & 0.00 & 0.00 & 0.00 & 0.00 & 0.00 & 0.00 & 0.00 \\
\hline V5 & 1.00 & 1.00 & 0.50 & 0.10 & 0.10 & 0.10 & 0.10 & 0.10 & 0.10 \\
\hline $\mathrm{v} 6$ & 1.00 & 1.00 & 0.70 & 0.00 & 0.00 & 0.00 & 0.00 & 0.00 & 0.00 \\
\hline HSI & 1.00 & 1.10 & 0.83 & & & & & & \\
\hline
\end{tabular}


Table C-9. Replace Column Number from Field Sheet With HSI Score.

\begin{tabular}{|c|c|c|c|c|c|c|c|c|c|}
\hline Serial Number & 71 & 73 & 83 & 1 & 3 & 14 & 39 & 65 & 67 \\
\hline \multirow[t]{2}{*}{ RAI Cover Type } & $\mathbf{F}$ & $\mathbf{F}$ & $\mathbf{F}$ & $\mathbf{G}$ & $\mathbf{G}$ & $\mathbf{G}$ & $\mathbf{G}$ & G & $\mathbf{G}$ \\
\hline & $\mathrm{XX}$ & $\mathrm{XX}$ & & & & & $i$ & $:$ & \\
\hline \multicolumn{10}{|l|}{ Meadowlark } \\
\hline V1 & 0.05 & 0.30 & 0.30 & 0.70 & 0.00 & 1.00 & 1.00 & 1.00 & 0.70 \\
\hline V2 & 0.10 & 0.20 & 0.30 & 0.70 & 0.30 & 0.30 & 1.00 & 1.00 & 0.70 \\
\hline V3 & 1.00 & 0.30 & 0.30 & 1.00 & 1.00 & 1.00 & 0.30 & 0.30 & 1.00 \\
\hline V4 & 0.00 & 0.00 & 0.00 & 0.00 & 0.25 & 0.75 & 0.25 & 0.25 & 0.75 \\
\hline V5 & 0.70 & 0.00 & 1.00 & 1.00 & 1.00 & 1.00 & 1.00 & 1.00 & 1.00 \\
\hline \multirow[t]{2}{*}{ HSI } & & & & 0.00 & 0.00 & 0.47 & 0.27 & 0.27 & 0.61 \\
\hline & & & & & & & 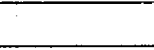 & & \\
\hline Chickadee & & & & & & & $:$ & & \\
\hline V1 & 0.70 & 1.00 & 0.70 & & & & $\vdots$ & & \\
\hline V2 & 0.70 & 1.00 & 1.00 & & & & 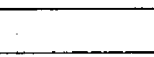 & & \\
\hline V3 & 1.00 & 0.50 & 1.00 & & & & $:$ & & \\
\hline \multirow[t]{2}{*}{ HSI } & 0.79 & 0.79 & 0.89 & & & & $\therefore$ & & \\
\hline & & & & & & 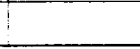 & & & \\
\hline \multicolumn{10}{|l|}{ Yellow Warbler } \\
\hline $\mathrm{V} 1$ & 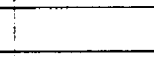 & $!$ & & 0.00 & & 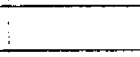 & 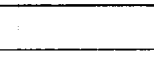 & & \\
\hline $\mathrm{V} 2$ & 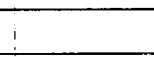 & 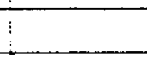 & & 0.00 & & 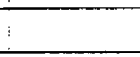 & $\vdots$ & & \\
\hline v3 & & $\because$ & & 0.10 & & & & & \\
\hline \multirow[t]{2}{*}{ HSI } & & & & 0.00 & & & & & \\
\hline & & 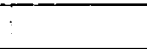 & & & & & & & \\
\hline \multicolumn{2}{|l|}{ Great Blue Heron } & $\vdots$ & & & & 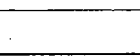 & & & \\
\hline $\mathrm{V} 1$ & 1.00 & 1.00 & 1.00 & 1.00 & 1.00 & 1.00 & 1.00 & 1.00 & 1.00 \\
\hline V2 & 0.10 & 0.10 & 0.10 & 0.10 & 0.10 & 0.30 & 0.30 & 0.50 & 0.30 \\
\hline V3 & 1.00 & 1.00 & 0.30 & 0.30 & 0.30 & 1.00 & 0.50 & 1.00 & 1.00 \\
\hline V4 & 0.00 & 1.00 & 0.00 & 0.00 & 0.00 & 0.00 & 0.00 & 0.00 & 0.00 \\
\hline V5 & 1.00 & 0.00 & 1.00 & 1.00 & 1.00 & 1.00 & 1.00 & 1.00 & 1.00 \\
\hline V6 & 1.00 & 1.00 & 1.00 & 1.00 & 1.00 & 1.00 & 1.00 & 1.00 & 1.00 \\
\hline \multirow[t]{2}{*}{ HSI } & 0.00 & 0.10 & 0.00 & 0.03 & 0.03 & 0.30 & 0.15 & 0.50 & 0.30 \\
\hline & & $!$ & & & & & & & \\
\hline \multicolumn{2}{|l|}{ Downy Woodpecke } & 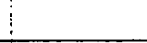 & & & & $\vdots$ & & & \\
\hline V1 & 1.00 & 1.00 & 1.00 & & & 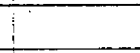 & & & \\
\hline V2 & 0.80 & 0.20 & 1.00 & & & 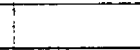 & & & \\
\hline \multirow[t]{3}{*}{ HSI } & 0.80 & 0.20 & 1.00 & & & $i$ & & & \\
\hline & 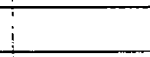 & & & & & 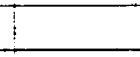 & & & \\
\hline & $i$ & 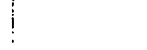 & & & & $\vdots$ & & & \\
\hline
\end{tabular}




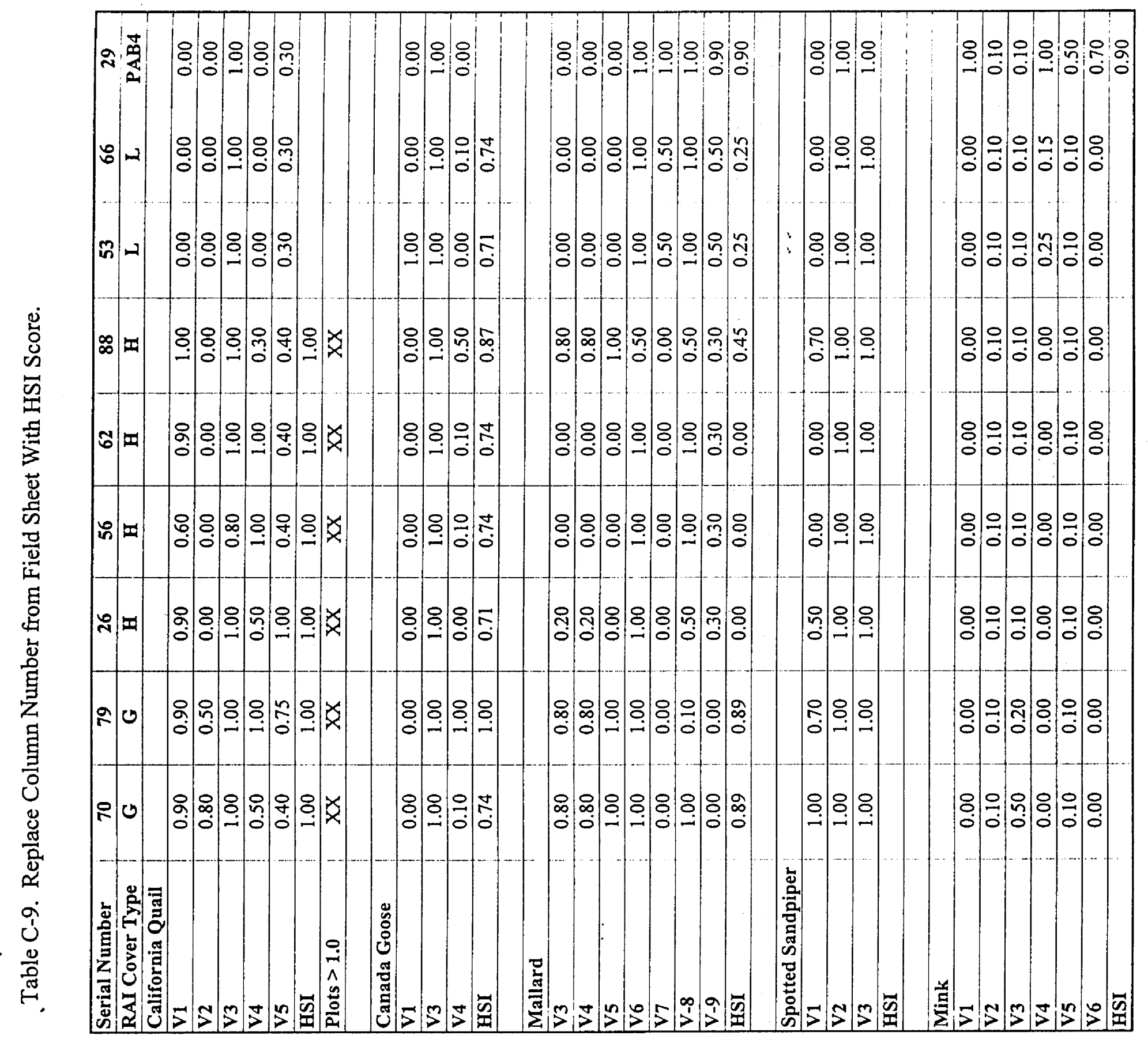


Table C-9. Replace Column Number from Field Sheet With HSI Score.

\begin{tabular}{|c|c|c|c|c|c|c|c|c|c|}
\hline Serial Number & 70 & 79 & 26 & 56 & 62 & 88 & 53 & 66 & 29 \\
\hline RAI Cover Type & $\mathbf{G}$ & $\mathbf{G}$ & $\mathbf{H}$ & $\mathbf{H}$ & $\mathbf{H}$ & $\mathbf{H}$ & $\mathbf{L}$ & $\mathbf{L}$ & PAB4 \\
\hline & & & & & & & & 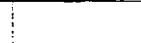 & \\
\hline \multicolumn{10}{|l|}{ Meadowlark } \\
\hline V1 & 1.00 & 1.00 & 1.00 & 0.30 & 1.00 & 0.70 & 0.00 & 0.00 & 0.00 \\
\hline V2 & 1.00 & 1.00 & 1.00 & 0.30 & 1.00 & 0.10 & 0.00 & 0.00 & 0.00 \\
\hline V3 & 0.00 & 0.30 & 0.30 & 1.00 & 1.00 & 0.30 & 0.00 & 0.00 & 0.00 \\
\hline V4 & 0.75 & 0.75 & 0.00 & 0.00 & 0.00 & 0.75 & 0.00 & 0.00 & 0.00 \\
\hline V5 & 0.20 & 0.70 & 1.00 & 1.00 & 1.00 & 1.00 & 1.00 & 1.00 & 1.00 \\
\hline \multirow[t]{2}{*}{ HSI } & 0.00 & 0.33 & & & & & & & \\
\hline & & & & & & & & & \\
\hline \multicolumn{10}{|l|}{ Chickadee } \\
\hline \multicolumn{10}{|l|}{ V1 } \\
\hline \multicolumn{10}{|l|}{ V2 } \\
\hline \multicolumn{10}{|l|}{ V3 } \\
\hline \multirow[t]{2}{*}{ HSI } & & & & & & & & $\vdots$ & \\
\hline & & & & & & & & 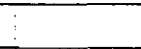 & \\
\hline \multicolumn{10}{|l|}{ Yellow Warbler } \\
\hline vi & & & & & & & & & \\
\hline \multicolumn{10}{|l|}{ v2 } \\
\hline $\bar{v} 3$ & & & & & & & & & \\
\hline \multirow[t]{2}{*}{ HSI } & & & & & & & & & \\
\hline & & & & & & & & & \\
\hline \multicolumn{10}{|l|}{ Great Blue Heron } \\
\hline V1 & 1.00 & 1.00 & 1.00 & 1.00 & 1.00 & 1.00 & 1.00 & 1.00 & 1.00 \\
\hline $\mathrm{V} 2$ & 0.30 & 0.30 & 0.10 & 0.50 & 0.50 & 0.10 & 0.20 & 0.40 & 0.70 \\
\hline $\mathrm{v} 3$ & 1.00 & 1.00 & 1.00 & 1.00 & 1.00 & 0.30 & 1.00 & 1.00 & 1.00 \\
\hline$\sqrt{\mathrm{V}} 4$ & 0.00 & 0.00 & 0.00 & 0.00 & 0.00 & 0.00 & 0.00 & 0.00 & 0.00 \\
\hline V5 & 0.50 & 1.00 & 0.00 & 1.00 & 1.00 & 1.00 & 1.00 & 1.00 & 1.00 \\
\hline V6 & 1.00 & 1.00 & 1.00 & 1.00 & 1.00 & 1.00 & 1.00 & 1.00 & 1.00 \\
\hline \multirow[t]{2}{*}{ HSI } & 0.30 & 0.30 & & & & & 0.20 & 0.40 & \\
\hline & & & & & & & & 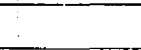 & \\
\hline \multicolumn{2}{|l|}{ Downy Woodpecke } & & & & & & & & \\
\hline v1 & & & & & & & & . & \\
\hline \multicolumn{10}{|l|}{$\sqrt{2}$} \\
\hline \multirow[t]{3}{*}{$\mathrm{HSI}$} & & & & & & & & $\vdots$ & \\
\hline & & & & & & & & & \\
\hline & & & & & & & & : & \\
\hline
\end{tabular}




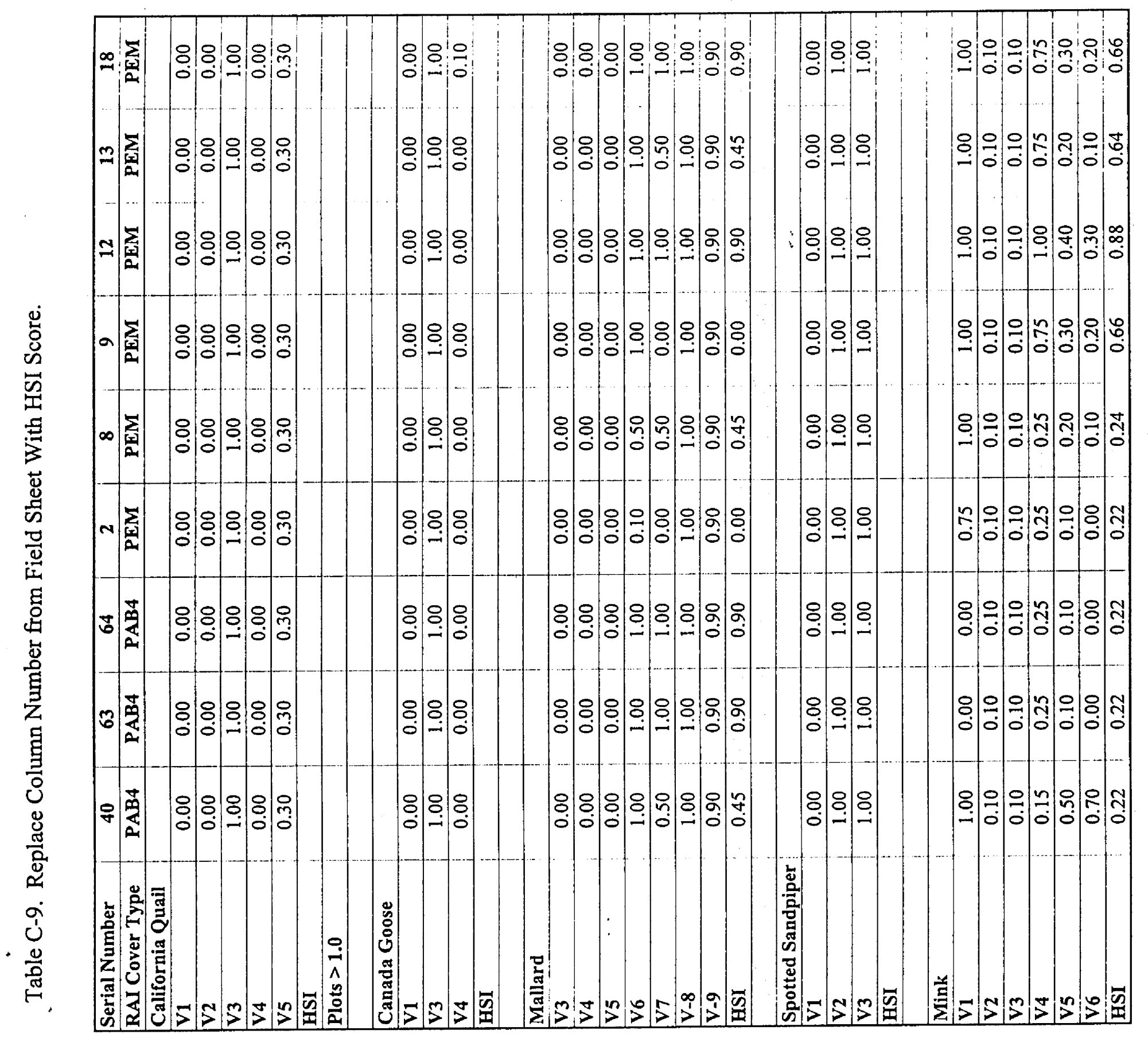


Table C-9. Replace Column Number from Field Sheet With HSI Score.

\begin{tabular}{|c|c|c|c|c|c|c|c|c|c|}
\hline Serial Number & 40 & 63 & 64 & 2 & 8 & 9 & 12 & 13 & 18 \\
\hline RAI Cover Type & PAB4 & PAB 4 & $\mathbf{P A B 4}$ & PEM & PEM & PEM & PEM & PEM & PEM \\
\hline \multicolumn{10}{|l|}{ Meadowlark } \\
\hline V1 & 0.00 & 0.00 & 0.00 & 0.00 & 0.00 & 0.00 & 0.00 & 0.00 & $0 . \overline{00}$ \\
\hline V2 & 0.00 & 0.00 & 0.00 & 0.00 & 0.00 & 0.00 & 0.00 & 0.00 & 0.00 \\
\hline V3 & 0.00 & 0.00 & 0.00 & 0.00 & 0.00 & 0.00 & 0.00 & 0.00 & 0.00 \\
\hline V4 & 0.00 & 0.00 & 0.00 & 0.00 & 0.00 & 0.00 & 0.00 & 0.00 & 0.00 \\
\hline V5 & 1.00 & 1.00 & 1.00 & 1.00 & 1.00 & 1.00 & 1.00 & 1.00 & 1.00 \\
\hline \multirow[t]{2}{*}{ HSI } & & & & & & & & & 0.00 \\
\hline & & & $\cdot$ & & & & & & \\
\hline Chickadee & & & & & & & & & \\
\hline \multicolumn{10}{|l|}{ V1 } \\
\hline V2 & & & & & & & & & \\
\hline V3 & & & & & & & & & \\
\hline \multirow[t]{2}{*}{ HSI } & & & & & & & & & \\
\hline & & & & & & & & & \\
\hline Yellow Warbler & & & & & & & & & \\
\hline v1 & & & & & & & & & \\
\hline $\mathbf{v}$ & & & & & & & & & \\
\hline$\sqrt{3} 3$ & & & & & & & & & \\
\hline \multicolumn{10}{|l|}{ HSI } \\
\hline & & & & & & & & & \\
\hline \multicolumn{10}{|l|}{ Great Blue Heron } \\
\hline $\mathrm{V} 1$ & 1.00 & 1.00 & 1.00 & 1.00 & 1.00 & 1.00 & 1.00 & 1.00 & 1.00 \\
\hline V2 & 1.00 & 0.70 & 0.70 & 0.70 & 0.70 & 0.50 & 1.00 & 0.70 & 1.00 \\
\hline V3 & 1.00 & 1.00 & 1.00 & 0.10 & 0.30 & 1.00 & 1.00 & 1.00 & 1.00 \\
\hline V4 & 0.00 & 0.00 & 0.00 & 0.00 & 0.00 & 0.00 & 0.00 & 0.00 & 0.00 \\
\hline V5 & 1.00 & 1.00 & 1.00 & 1.00 & 1.00 & 1.00 & 1.00 & 1.00 & 1.00 \\
\hline$\sqrt{6}$ & 1.00 & 1.00 & 1.00 & 1.00 & 1.00 & 1.00 & 1.00 & 1.00 & 1.00 \\
\hline \multirow[t]{2}{*}{ HSI } & & & & & & & & & \\
\hline & & & & & & & & & \\
\hline \multicolumn{2}{|l|}{ Downy Woodpecke } & & & & & & & & \\
\hline \multicolumn{10}{|l|}{ V1 } \\
\hline $\mathrm{V} 2$ & & & & & & & & & \\
\hline \multirow[t]{3}{*}{ HSI } & & & & & & & 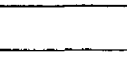 & & \\
\hline & & & & & & & 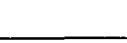 & & \\
\hline & & & & & & & 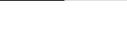 & & \\
\hline
\end{tabular}




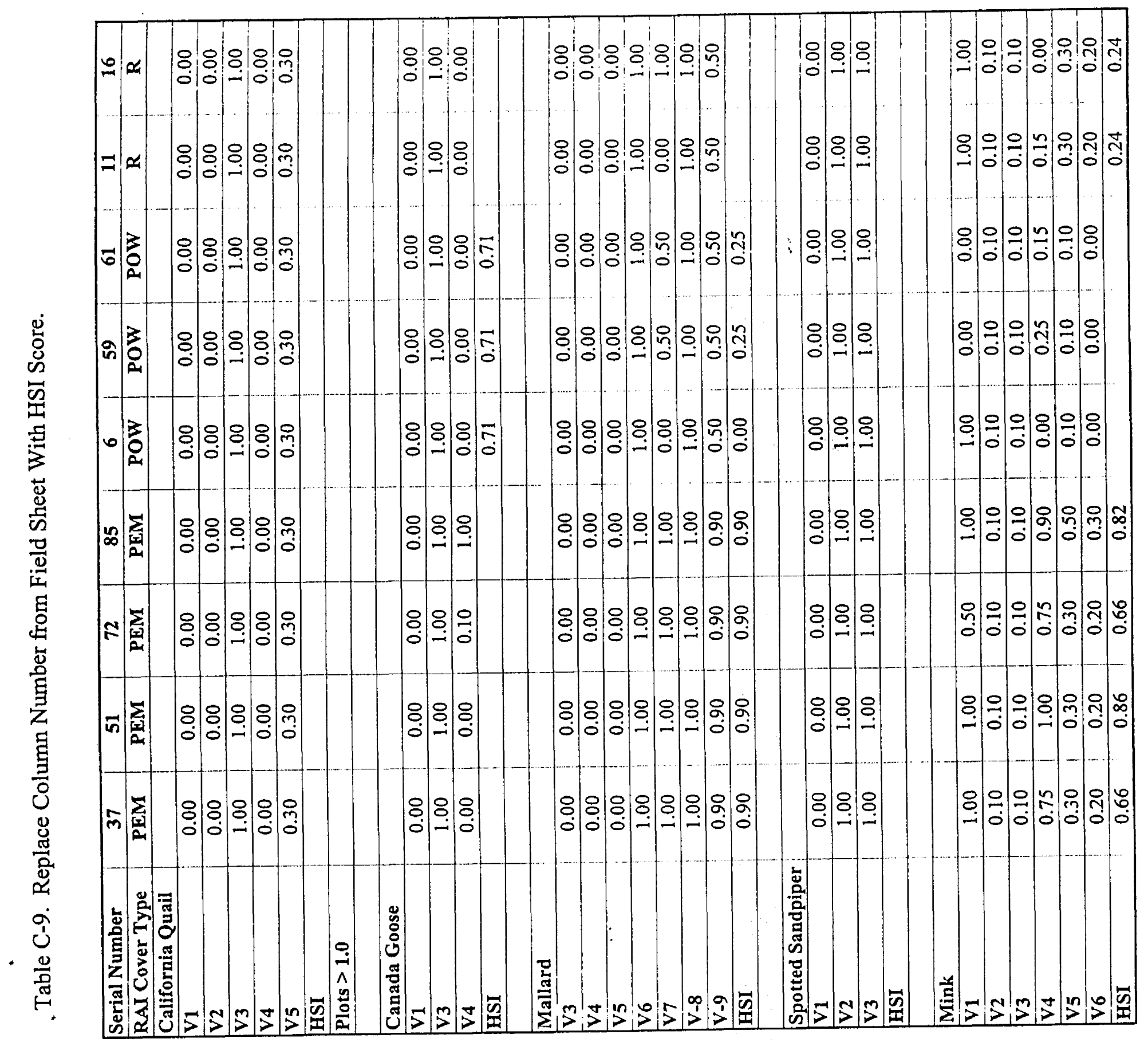


Table C-9. Replace Column Number from Field Sheet With HSI Score.

\begin{tabular}{|c|c|c|c|c|c|c|c|c|c|}
\hline Serial Number & 37 & 51 & 72 & 85 & 6 & 59 & 61 & 11 & 16 \\
\hline RAI Cover Type & PEM & PEM & PEM & PEM & POW & POW & POW & $\mathbf{R}$ & $\mathbf{R}$ \\
\hline & & & & & & & & & \\
\hline \multicolumn{10}{|l|}{ Meadowlark } \\
\hline $\mathrm{V} 1$ & 0.00 & 0.00 & 0.00 & 0.00 & 0.00 & 0.00 & 0.00 & 0.00 & 0.00 \\
\hline V2 & 0.00 & 0.00 & 0.00 & 0.00 & 0.00 & 0.00 & 0.00 & 0.00 & 0.00 \\
\hline V3 & 0.00 & 0.00 & 0.00 & 0.00 & 0.00 & 0.00 & 0.00 & 0.00 & 0.00 \\
\hline V4 & 0.00 & 0.00 & 0.00 & 0.00 & 0.00 & 0.00 & 0.00 & 0.00 & 0.00 \\
\hline V5 & 1.00 & 1.00 & 1.00 & 1.00 & 1.00 & 1.00 & 1.00 & 1.00 & 1.00 \\
\hline \multicolumn{10}{|l|}{ HSI } \\
\hline & & & & & & & & & \\
\hline \multicolumn{10}{|l|}{ Chickadee } \\
\hline V1 & 1 & & & & & 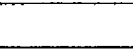 & $\frac{1}{1}$ & 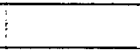 & \\
\hline V2 & 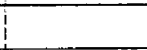 & & & & & & 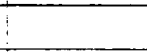 & & \\
\hline V3 & & & & & & & & 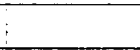 & \\
\hline \multicolumn{10}{|l|}{ HSI } \\
\hline & & & & & & & 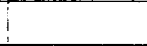 & & \\
\hline \multicolumn{10}{|l|}{ Yellow Warbler } \\
\hline v1 & & & & & & & 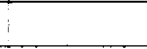 & & \\
\hline $\mathrm{V} 2$ & & & & & & & 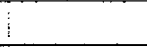 & & \\
\hline$\sqrt{3}$ & & & & & & & 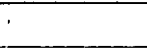 & & \\
\hline \multirow[t]{2}{*}{ HSI } & & & & & & & & & \\
\hline & & & & & & & & & \\
\hline \multicolumn{10}{|l|}{ Great Blue Heron } \\
\hline V1 & 1.00 & 1.00 & 1.00 & 1.00 & 1.00 & 1.00 & 1.00 & 1.00 & 1.00 \\
\hline V2 & 1.00 & 0.70 & 1.00 & 0.70 & 0.70 & 0.10 & 0.10 & 0.70 & 0.70 \\
\hline V3 & 1.00 & 0.50 & 0.50 & 1.00 & 1.00 & 1.00 & 1.00 & 1.00 & 1.00 \\
\hline V4 & 0.00 & 0.00 & 0.00 & 0.00 & 0.00 & 0.00 & 0.00 & 0.00 & 0.00 \\
\hline V5 & 1.00 & 1.00 & 0.50 & 1.00 & 1.00 & 0.00 & 0.00 & 1.00 & 1.00 \\
\hline $\mathrm{V} 6$ & 1.00 & 1.00 & 1.00 & 1.00 & 1.00 & 1.00 & 1.00 & 1.00 & 1.00 \\
\hline \multirow[t]{2}{*}{ HSI } & 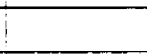 & & & & 0.70 & 0.10 & 0.10 & 0.70 & 0.70 \\
\hline & & & & & & & & & \\
\hline \multicolumn{10}{|l|}{ Downy Woodpecke } \\
\hline V1 & & & & & & & & & \\
\hline $\mathbf{v} 2$ & i & & & & & 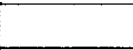 & & & \\
\hline \multicolumn{10}{|l|}{ HSI } \\
\hline & & & & & & 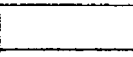 & $!$ & 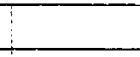 & \\
\hline & & & & & & 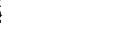 & & $!$ & \\
\hline
\end{tabular}


- Table C-9. Replace Column Number from Field Sheet With HSI Score.

\begin{tabular}{|c|c|c|c|c|c|c|c|c|c|}
\hline Serial Number & 36 & 43 & 47 & 75 & 76 & 87 & 19 & 30 & 48 \\
\hline RAI Cover Type & $\mathbf{R}$ & $\mathbf{R}$ & $\mathbf{R}$ & $\mathbf{R}$ & $\mathbf{R}$ & $\mathbf{R}$ & $\mathbf{S}$ & $\mathbf{S}$ & $\mathrm{s}$ \\
\hline California Quail & & & & & & & 1 & 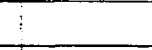 & \\
\hline V1 & 0.00 & 0.00 & 0.00 & 0.00 & 0.00 & 0.00 & 0.30 & 0.90 & $0 . \overline{30}$ \\
\hline $\mathrm{V} 2$ & 0.00 & 0.00 & 0.00 & 0.00 & 0.00 & 0.00 & 1.00 & 1.00 & 0.80 \\
\hline V3 & 1.00 & 1.00 & 1.00 & 1.00 & 1.00 & 1.00 & 1.00 & 1.00 & 1.00 \\
\hline V4 & 0.00 & 0.00 & 0.00 & 0.00 & 0.00 & 0.00 & 0.50 & 0.00 & 0.50 \\
\hline V5 & 0.30 & 0.30 & 0.30 & 0.30 & 0.30 & 0.30 & 0.75 & 0.30 & 0.75 \\
\hline HSI & & & & & & & 1.00 & 1.00 & 1.00 \\
\hline \multirow{2}{*}{ Plots $>1.0$} & & & & & & & $\mathrm{XX}$ & $\mathrm{XX}$ & $x \mathrm{x}$ \\
\hline & & & & & & & & & \\
\hline \multicolumn{10}{|l|}{ Canada Goose } \\
\hline V1 & 0.00 & 0.00 & 0.00 & 0.00 & 0.00 & 0.00 & 0.00 & 0.00 & $0 . \overline{00}$ \\
\hline V3 & 1.00 & 1.00 & 1.00 & 1.00 & 1.00 & 1.00 & 1.00 & 1.00 & 1.00 \\
\hline V4 & 0.10 & 0.00 & 0.00 & 0.10 & 1.00 & 0.00 & 1.00 & 0.00 & 0.00 \\
\hline \multirow[t]{2}{*}{ HSI } & & & & & & & 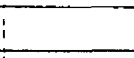 & & \\
\hline & & & & & & & 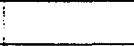 & & \\
\hline \multicolumn{10}{|l|}{ Mallard } \\
\hline V3 & 0.00 & 0.00 & 0.00 & 0.00 & 0.00 & 0.00 & 0.50 & 0.00 & 0.20 \\
\hline V4 & 0.00 & 0.00 & 0.00 & 0.00 & 0.00 & 0.00 & 0.50 & 0.00 & 0.20 \\
\hline V5 & 0.00 & 0.00 & 0.00 & 0.00 & 0.00 & 0.00 & 0.00 & 0.00 & 0.30 \\
\hline V6 & 1.00 & 1.00 & 1.00 & 1.00 & 1.00 & $\overline{0.50}$ & 1.00 & 1.00 & 1.00 \\
\hline V7 & 0.00 & 0.00 & 0.00 & 0.50 & 0.00 & 0.00 & 0.00 & 0.00 & 0.00 \\
\hline $\mathrm{V}-8$ & 1.00 & 1.00 & 1.00 & 1.00 & 1.00 & 1.00 & 1.00 & 1.00 & 1.00 \\
\hline V-9 & 0.50 & 0.50 & 0.50 & 0.50 & 0.50 & 0.50 & 0.00 & 0.00 & 0.00 \\
\hline \multicolumn{10}{|l|}{ HSI } \\
\hline & & & & & & & & & \\
\hline Spotted Sandpiper & & & & & & & $\cdots$ & & \\
\hline V1 & 0.00 & 0.00 & 0.00 & 0.00 & 0.00 & 0.00 & 0.00 & 1.00 & 0.50 \\
\hline V2 & 1.00 & 1.00 & 1.00 & 1.00 & 1.00 & 1.00 & 1.00 & 1.00 & 1.00 \\
\hline V3 & 1.00 & 1.00 & 1.00 & 1.00 & 1.00 & 1.00 & 1.00 & 1.00 & 1.00 \\
\hline \multirow[t]{2}{*}{ HSI } & & & & & & & i & & \\
\hline & & & & & & & i & & \\
\hline \multicolumn{10}{|l|}{ Mink } \\
\hline V1 & 1.00 & 1.00 & 1.00 & 1.00 & 1.00 & 1.00 & 1.00 & 0.75 & 1.00 \\
\hline$\sqrt{2}$ & 0.10 & 0.10 & 0.10 & 0.10 & 0.10 & 0.10 & 0.10 & 0.10 & 0.10 \\
\hline $\mathrm{v} 3$ & 0.10 & 0.10 & 0.10 & 0.10 & 0.10 & 0.10 & 0.75 & 1.00 & 0.75 \\
\hline V4 & 0.10 & 0.00 & 0.15 & 0.15 & 0.00 & 0.15 & 0.00 & 0.00 & 0.00 \\
\hline v 5 & 0.40 & 0.50 & 0.40 & 0.50 & 0.30 & 0.30 & 1.00 & 0.40 & 0.30 \\
\hline V6 & 0.30 & 0.70 & 0.30 & 0.70 & 0.30 & 0.30 & 1.00 & 0.30 & 0.30 \\
\hline HSI & 0.35 & 0.59 & 0.35 & 0.59 & 0.30 & 0.30 & 0.93 & 0.75 & 0.58 \\
\hline
\end{tabular}


. Table C-9. Replace Column Number from Field Sheet With HSI Score.

\begin{tabular}{|c|c|c|c|c|c|c|c|c|c|}
\hline Serial Number & 36 & 43 & 47 & 75 & 76 & 87 & 19 & 30 & 48 \\
\hline RAI Cover Type & $\mathbf{R}$ & $\mathbf{R}$ & $\mathbf{R}$ & $\mathbf{R}$ & $\mathbf{R}$ & $\mathbf{R}$ & $\mathbf{S}$ & $\mathbf{S}$ & $\mathbf{S}$ \\
\hline & & & & & & & & & \\
\hline Meadowlark & & & & & & & & & \\
\hline V1 & 0.00 & 0.00 & 0.00 & 0.00 & 0.00 & 0.00 & 0.10 & 1.00 & 0.10 \\
\hline V2 & 0.00 & 0.00 & 0.00 & 0.00 & 0.00 & 0.00 & 0.20 & 0.70 & 0.20 \\
\hline V3 & 0.00 & 0.00 & 0.00 & 0.00 & 0.00 & 0.00 & 0.00 & 0.30 & 1.00 \\
\hline V4 & 0.00 & 0.00 & 0.00 & 0.00 & 0.00 & 0.00 & 0.00 & 0.00 & 0.25 \\
\hline V5 & 1.00 & 1.00 & 1.00 & 1.00 & 1.00 & 1.00 & 0.00 & 0.00 & 0.00 \\
\hline \multicolumn{10}{|l|}{ HSI } \\
\hline & & & & & & & & & \\
\hline \multicolumn{10}{|l|}{ Chickadee } \\
\hline \multicolumn{10}{|l|}{$\mathrm{V} 1$} \\
\hline \multicolumn{10}{|l|}{ V2 } \\
\hline \multicolumn{10}{|l|}{ V3 } \\
\hline HSI & & & & & & & & & \\
\hline & & & & & & & & & \\
\hline \multicolumn{10}{|l|}{ Yellow Warbler } \\
\hline V1 & & & & & & & 1.00 & 0.70 & 1.00 \\
\hline$\sqrt{2}$ & & & & & & & 1.00 & 1.00 & 1.00 \\
\hline v3 & & & & & & & 0.60 & 0.15 & 0.40 \\
\hline HSI & & & & & & & 0.84 & 0.47 & 0.74 \\
\hline & & & & & & & & & $-\ldots$ \\
\hline \multicolumn{10}{|l|}{ Great Blue Heron } \\
\hline V1 & 1.00 & 1.00 & 1.00 & 1.00 & 1.00 & 1.00 & 1.00 & 1.00 & 1.00 \\
\hline $\mathbf{V} \mathbf{2}$ & 1.00 & 1.00 & 0.70 & 1.00 & 1.00 & 1.00 & 0.10 & 0.10 & 0.30 \\
\hline V3 & 1.00 & 1.00 & 1.00 & 1.00 & 1.00 & 0.30 & 1.00 & 1.00 & 1.00 \\
\hline V4 & 0.00 & 0.00 & 0.00 & 0.00 & 0.00 & 0.00 & $0: 00$ & 0.00 & 0.00 \\
\hline V5 & 1.00 & 1.00 & 1.00 & 0.50 & 1.00 & 1.00 & 1.00 & 1.00 & 1.00 \\
\hline V6 & 1.00 & 1.00 & 1.00 & 1.00 & 1.00 & 1.00 & 1.00 & 1.00 & 1.00 \\
\hline \multirow[t]{2}{*}{ HSI } & 1.00 & 1.00 & 0.70 & 1.00 & 1.00 & 0.30 & & & \\
\hline & & & & & & & & & \\
\hline \multicolumn{10}{|l|}{ Downy Woodpecke } \\
\hline V1 & & & & & & & & & \\
\hline \multicolumn{10}{|l|}{ V2 } \\
\hline \multicolumn{10}{|l|}{ HSI } \\
\hline & & & & & & & & & \\
\hline 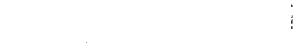 & & & & & & & & & \\
\hline
\end{tabular}


Table C-9. Replace Column Number from Field Sheet With HSI Score.

\begin{tabular}{|c|c|c|c|c|c|c|c|c|c|}
\hline Serial Number & 57 & 60 & 68 & 77 & 84 & 27 & 35 & 42 & 50 \\
\hline RAI Cover Type & $\mathbf{S}$ & $\mathbf{S}$ & $\mathbf{S}$ & $\mathbf{S}$ & $\mathbf{S}$ & SGCM & SGCM & SGCM & SGCM \\
\hline \multicolumn{10}{|l|}{ California Quail } \\
\hline V1 & 0.30 & 0.30 & 1.00 & 0.30 & 0.30 & 0.00 & 0.10 & 0.00 & 0.60 \\
\hline V2 & 0.50 & 0.80 & 0.40 & 0.80 & 1.00 & 0.00 & 0.00 & 0.00 & 0.40 \\
\hline V3 & 1.00 & 1.00 & 1.00 & 1.00 & 1.00 & 1.00 & 1.00 & 1.00 & 0.80 \\
\hline V4 & 1.00 & 0.50 & 0.50 & 0.00 & 0.50 & 0.00 & 0.00 & 0.00 & 1.00 \\
\hline V5 & 0.40 & 0.40 & 0.40 & 0.30 & 0.40 & 0.30 & 0.30 & 0.30 & 1.00 \\
\hline HSI & 1.00 & 1.00 & 1.00 & 1.00 & 1.00 & & & & \\
\hline Plots $>1.0$ & $\mathrm{XX}$ & $\mathrm{XX}$ & $\mathrm{XX}$ & $\mathrm{XX}$ & $\mathrm{XX}$ & & & & \\
\hline & & & & & & & & & \\
\hline \multicolumn{10}{|l|}{ Canada Goose } \\
\hline V1 & 0.00 & 0.00 & 0.00 & 0.00 & 0.00 & 0.00 & 0.00 & 0.00 & 0.00 \\
\hline $\mathrm{V3}$ & 1.00 & 1.00 & 1.00 & 1.00 & 1.00 & 1.00 & 1.00 & 1.00 & 1.00 \\
\hline V4 & 0.10 & 0.00 & 0.10 & 1.00 & 1.00 & 0.00 & 0.00 & 0.00 & 0.00 \\
\hline HSI & & & & & & 0.71 & 0.71 & 0.71 & 0.71 \\
\hline & & & & & & & & & \\
\hline \multicolumn{10}{|l|}{ Mallard } \\
\hline V3 & 1.00 & 0.00 & 0.20 & 0.00 & 0.10 & 0.00 & 0.00 & 0.00 & 0.20 \\
\hline $\mathbf{V 4}$ & 1.00 & 0.00 & 0.20 & 0.00 & 0.10 & 0.00 & 0.00 & 0.00 & 0.20 \\
\hline V5 & 0.00 & 0.00 & 1.00 & 0.00 & 0.00 & 0.00 & 0.00 & 0.00 & 0.30 \\
\hline V6 & 1.00 & 1.00 & 1.00 & 1.00 & 1.00 & 1.00 & 1.00 & 1.00 & 1.00 \\
\hline$\sqrt{7}$ & 0.00 & 0.00 & 0.00 & 0.00 & 0.00 & 0.00 & 0.00 & 0.00 & 0.00 \\
\hline $\mathrm{V}-8$ & 1.00 & 1.00 & 1.00 & 1.00 & 1.00 & 1.00 & 1.00 & 1.00 & 1.00 \\
\hline V-9 & 0.00 & 0.00 & 0.00 & 0.00 & 0.00 & 0.30 & 0.30 & 0.30 & 0.30 \\
\hline \multirow[t]{2}{*}{ HSI } & & & & & & 0.00 & 0.00 & 0.00 & 0.24 \\
\hline & & & & & & & & & \\
\hline Spotted Sandpiper & & & & & & & $\cdots$ & & \\
\hline V1 & 0.20 & 1.00 & 0.00 & 0.70 & 0.20 & 0.00 & 0.20 & 0.00 & 0.00 \\
\hline V2 & 1.00 & 1.00 & 1.00 & 1.00 & 1.00 & 1.00 & 1.00 & 1.00 & 1.00 \\
\hline V3 & 1.00 & 1.00 & 1.00 & 1.00 & 1.00 & 1.00 & 1.00 & 1.00 & 1.00 \\
\hline \multirow[t]{2}{*}{ HSI } & & & & & & 0.67 & 0.73 & 0.67 & 0.67 \\
\hline & & & & & & & & & \\
\hline \multicolumn{10}{|l|}{ Mink } \\
\hline $\mathbf{v} 1$ & 1.00 & 0.00 & 0.50 & 1.00 & 1.00 & 0.00 & 0.00 & 0.00 & 0.00 \\
\hline $\mathrm{V} 2$ & 0.10 & 0.10 & 0.10 & 0.10 & 0.10 & 0.10 & 0.10 & 0.10 & 0.10 \\
\hline v3 & 1.00 & 0.75 & 1.00 & 0.50 & 1.00 & 0.10 & 0.10 & 0.10 & 0.20 \\
\hline$\sqrt{\mathrm{V}} 4$ & 0.00 & 0.00 & 0.00 & 0.00 & 0.00 & 0.15 & 0.00 & 0.00 & 0.00 \\
\hline V5 & 0.50 & 0.10 & 1.00 & 0.30 & 0.50 & 0.10 & 0.10 & 0.10 & 0.10 \\
\hline V6 & 0.30 & 0.00 & 1.00 & 0.30 & 0.30 & 0.00 & 0.00 & 0.00 & 0.00 \\
\hline HSI & 0.80 & 0.48 & 1.00 & 0.45 & 0.80 & 0.00 & 0.00 & 0.00 & 0.00 \\
\hline
\end{tabular}


Table C-9. Replace Column Number from Field Sheet With HSI Score.

\begin{tabular}{|c|c|c|c|c|c|c|c|c|c|}
\hline Serial Number & 57 & 60 & 68 & 77 & 84 & 27 & 35 & 42 & 50 \\
\hline \multirow[t]{2}{*}{ RAI Cover Type } & $\mathbf{S}$ & $\mathbf{S}$ & $\mathbf{S}$ & $\mathbf{S}$ & $\mathbf{S}$ & SGCM & SGCM & SGCM & SGCM \\
\hline & & & $\mathrm{XX}$ & & & & & & \\
\hline \multicolumn{10}{|l|}{ Meadowlark } \\
\hline V1 & 0.10 & 0.10 & 0.70 & 0.10 & 0.10 & 0.00 & 0.05 & 0.00 & 0.30 \\
\hline V2 & 0.20 & 0.20 & 0.30 & 0.30 & 0.20 & 0.00 & 0.00 & 0.00 & 0.30 \\
\hline V3 & 0.00 & 0.00 & 0.30 & 0.30 & 0.30 & 0.00 & 1.00 & 0.00 & 1.00 \\
\hline V4 & 0.00 & 0.00 & 0.75 & 0.00 & 0.00 & 0.00 & 0.00 & 0.00 & 0.25 \\
\hline V5 & 0.00 & 0.00 & 0.00 & 0.20 & 0.00 & 1.00 & 1.00 & 1.00 & 0.70 \\
\hline \multirow[t]{2}{*}{ HSI } & & & & & & & & & \\
\hline & & & & & & & & & \\
\hline Chickadee & & & & & & & & & \\
\hline \multicolumn{10}{|l|}{ V1 } \\
\hline \multicolumn{10}{|l|}{$\mathrm{V} 2$} \\
\hline \multicolumn{10}{|l|}{$\mathrm{V3}$} \\
\hline \multicolumn{10}{|l|}{ HSI } \\
\hline & & & & & & & & & \\
\hline \multicolumn{10}{|l|}{ Yellow Warbler } \\
\hline V1 & 0.70 & 1.00 & 0.70 & 0.50 & 0.70 & & & & \\
\hline $\mathrm{V} 2$ & 0.50 & 1.00 & 0.25 & 1.00 & 1.00 & & & & \\
\hline $\mathrm{V} 3$ & 0.25 & 0.40 & 0.60 & 0.25 & 0.10 & & & & \\
\hline \multirow[t]{2}{*}{ HSI } & 0.44 & 0.74 & 0.47 & 0.50 & 0.41 & & & & \\
\hline & & & & & & & & & \\
\hline \multicolumn{10}{|l|}{ Great Blue Heron } \\
\hline V1 & 1.00 & 1.00 & 1.00 & 1.00 & 1.00 & 1.00 & 1.00 & 1.00 & 1.00 \\
\hline V2 & 0.10 & 0.10 & 0.10 & 1.00 & 0.30 & 1.00 & 0.10 & 0.10 & 0.30 \\
\hline$\sqrt{3}$ & 1.00 & 1.00 & 1.00 & 1.00 & 1.00 & 1.00 & 1.00 & 1.00 & 1.00 \\
\hline V4 & 0.00 & 0.00 & 0.00 & 0.00 & 0.00 & 0.00 & $0: 00$ & 0.00 & 0.00 \\
\hline V5 & 1.00 & 1.00 & 1.00 & 1.00 & 1.00 & 1.00 & 1.00 & 0.00 & 1.00 \\
\hline V6 & 1.00 & 1.00 & 1.00 & 1.00 & 1.00 & 1.00 & 1.00 & 1.00 & 1.00 \\
\hline \multirow[t]{2}{*}{ HSI } & & 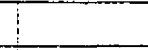 & & & & 1.00 & 0.10 & 0.10 & 0.30 \\
\hline & & $\vdots$ & & & & 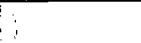 & & & \\
\hline \multicolumn{2}{|l|}{ Downy Woodpecke } & & & & & & & & \\
\hline \multicolumn{10}{|l|}{\begin{tabular}{|l|}
$\mathrm{v1}$ \\
\end{tabular}} \\
\hline \multicolumn{10}{|l|}{ V2 } \\
\hline \multicolumn{10}{|l|}{$\mathrm{H} S \mathrm{SI}$} \\
\hline i & & 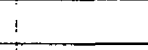 & & & & & & & \\
\hline : & & & & & & & & & \\
\hline
\end{tabular}


. Table C-9. Replace Column Number from Field Sheet With HSI Score.

\begin{tabular}{|c|c|c|c|c|c|c|c|}
\hline Serial Number & 78 & 21 & 31 & 33 & 38 & 74 & 86 \\
\hline RAI Cover Type & SGCM & SSG & SSG & SSG & SSG & SSG & SSG \\
\hline \multicolumn{8}{|l|}{ California Quail } \\
\hline $\mathrm{V} 1$ & 0.00 & 0.60 & 0.30 & 0.30 & 0.60 & 1.00 & 0.30 \\
\hline V2 & 0.00 & 0.40 & 0.40 & 0.40 & $0 . \overline{40}$ & 0.40 & 0.50 \\
\hline $\mathbf{V} 3$ & 1.00 & 1.00 & 0.50 & 0.30 & 1.00 & 1.00 & 0.10 \\
\hline V4 & 0.00 & 1.00 & 1.00 & 0.50 & 0.30 & 0.30 & 0.30 \\
\hline V5 & 0.30 & 0.75 & 0.60 & 0.10 & 0.40 & 0.75 & 0.40 \\
\hline HSI & & 1.00 & 1.00 & 0.95 & 1.00 & 1.00 & 1.00 \\
\hline \multirow[t]{2}{*}{ Plots $>1.0$} & & $\mathrm{XX}$ & $\mathrm{XX}$ & & $\mathrm{XX}$ & $\mathrm{XX}$ & $\mathrm{XX}$ \\
\hline & & 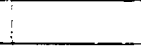 & & & & & \\
\hline Canada Goose & & & & 4 & & & ( \\
\hline V1 & 0.00 & 0.00 & 0.00 & 0.00 & 0.00 & 0.00 & 0.00 \\
\hline V3 & 1.00 & 1.00 & 1.00 & 1.00 & 1.00 & 1.00 & 1.00 \\
\hline V4 & 1.00 & 1.00 & 0.00 & 0.50 & 0.00 & 0.10 & 1.00 \\
\hline \multirow[t]{2}{*}{ HSI } & 1.00 & 1.00 & 0.71 & 0.87 & 0.71 & 0.74 & 1.00 \\
\hline & & $i$ & & & & & 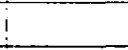 \\
\hline \multicolumn{8}{|l|}{ Mallard } \\
\hline v3 & 0.00 & 0.20 & 0.10 & 0.20 & 0.20 & 0.10 & 0.20 \\
\hline $\mathrm{V} 4$ & 0.00 & 0.20 & 0.10 & 0.20 & 0.20 & 0.10 & 0.20 \\
\hline v5 & 0.00 & 0.40 & 0.40 & 1.00 & 1.00 & 1.00 & 1.00 \\
\hline V6 & 1.00 & 0.50 & 1.00 & 1.00 & 1.00 & 1.00 & 1.00 \\
\hline $\mathrm{V} 7$ & 0.00 & 0.00 & 0.00 & 0.00 & 0.00 & 0.00 & 0.00 \\
\hline V-8 & 1.00 & 1.00 & 1.00 & 0.50 & 1.00 & 1.00 & 0.50 \\
\hline $\mathrm{V}-9$ & 0.30 & 0.00 & 0.00 & 0.00 & 0.00 & 0.00 & 0.00 \\
\hline \multirow[t]{2}{*}{ HSI } & 0.00 & 0.14 & 0.20 & 0.45 & 0.45 & 0.32 & 0.45 \\
\hline & & $\vdots$ & & & & & \\
\hline Spotted Sandpiper & & 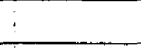 & & & & & $\because$ \\
\hline V1 & 0.00 & 0.00 & 0.50 & 0.00 & 0.00 & 0.00 & 0.50 \\
\hline V2 & 1.00 & 1.00 & 1.00 & 1.00 & 1.00 & 1.00 & 1.00 \\
\hline V3 & 1.00 & 1.00 & 1.00 & 1.00 & 1.00 & 1.00 & 1.00 \\
\hline \multirow[t]{2}{*}{ HSI } & 0.67 & $i$ & & & & & \\
\hline & & & & & & & \\
\hline \multicolumn{8}{|l|}{ Mink } \\
\hline $\mathrm{Vi}$ & 0.00 & 0.00 & 0.00 & 0.00 & 0.00 & 0.00 & 0.00 \\
\hline $\mathrm{V} 2$ & 0.10 & 0.10 & 0.10 & 0.10 & 0.10 & 0.10 & 0.10 \\
\hline V3 & 0.10 & 0.75 & 0.20 & 0.50 & 0.50 & 0.20 & 0.50 \\
\hline $\mathbf{V} 4$ & 0.00 & 0.00 & 0.00 & 0.00 & 0.00 & 0.00 & 0.00 \\
\hline V5 & 0.10 & 0.10 & 0.10 & 0.10 & 0.10 & 0.10 & 0.10 \\
\hline V6 & 0.00 & 0.00 & 0.00 & 0.00 & 0.00 & 0.00 & 0.00 \\
\hline $\mathrm{HSI}$ & 0.00 & & & & & & \\
\hline
\end{tabular}


. Table C-9. Replace Column Number from Field Sheet With HSI Score.

\begin{tabular}{|c|c|c|c|c|c|c|c|}
\hline Serial Number & 78 & 21 & 31 & 33 & 38 & 74 & 86 \\
\hline RAI Cover Type & SGCM & SSG & SSG & SSG & SSG & SSG & SSG \\
\hline \multicolumn{8}{|l|}{ Meadowlark } \\
\hline V1 & 0.00 & 0.30 & 0.10 & 0.10 & 0.30 & 0.70 & 0.10 \\
\hline $\mathbf{V} 2$ & 0.00 & 0.30 & 0.20 & 0.20 & 0.30 & 0.70 & 0.20 \\
\hline $\mathrm{V3}$ & 0.00 & 0.30 & 1.00 & 1.00 & 0.30 & 1.00 & 1.00 \\
\hline V4 & 0.00 & 1.00 & 1.00 & 0.75 & 0.75 & 0.75 & 0.75 \\
\hline V5 & 1.00 & 0.00 & 0.70 & 0.20 & 0.20 & 0.70 & 0.20 \\
\hline HSI & & 0.00 & 0.10 & 0.02 & 0.03 & 0.42 & 0.02 \\
\hline & & & & & & & \\
\hline \multicolumn{8}{|l|}{ Chickadee } \\
\hline \multicolumn{8}{|l|}{ V1 } \\
\hline \multicolumn{8}{|l|}{$\mathbf{v 2}$} \\
\hline \multicolumn{8}{|l|}{ V3 } \\
\hline \multicolumn{8}{|l|}{ HSI } \\
\hline & i & & & & & & \\
\hline Yellow Warbler & 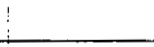 & & & & & & \\
\hline V1 & 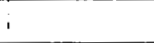 & 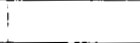 & & & & & \\
\hline V2 & $:$ & 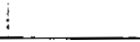 & & & & & \\
\hline \multicolumn{8}{|l|}{ V3 } \\
\hline \multicolumn{8}{|l|}{ HSI } \\
\hline & 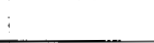 & 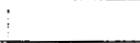 & & & & & \\
\hline \multicolumn{8}{|l|}{ Great Blue Heron } \\
\hline V1 & 1.00 & 1.00 & 1.00 & 1.00 & 1.00 & 1.00 & 1.00 \\
\hline V2 & 1.00 & 0.30 & 0.10 & 0.30 & 0.30 & 0.30 & 0.30 \\
\hline V3 & 1.00 & 0.30 & 1.00 & 1.00 & 1.00 & 1.00 & 0.50 \\
\hline V4 & 0.00 & 0.00 & 0.00 & 0.00 & 0.00 & 0.00 & 0.00 \\
\hline V5 & 1.00 & 1.00 & 0.00 & 1.00 & 1.00 & 0.50 & 1.00 \\
\hline V6 & 1.00 & 1.00 & 1.00 & 1.00 & 1.00 & 1.00 & 1.00 \\
\hline \multirow[t]{2}{*}{ HSI } & 1.00 & 0.09 & 0.10 & 0.30 & $0 . \overline{30}$ & 0.30 & 0.15 \\
\hline & & 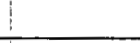 & & & & & \\
\hline \multicolumn{8}{|l|}{ Downy Woodpecke: } \\
\hline $\mathrm{V} 1$ & 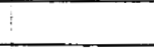 & 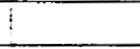 & & & & & \\
\hline V2 & & 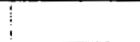 & & & & & \\
\hline \multicolumn{8}{|l|}{$\mathrm{HSI}$} \\
\hline & 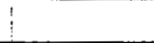 & ! & & & & & \\
\hline & i & ! & & & & & \\
\hline
\end{tabular}


Table C-10. Yakama Method with HSI Scores replacing step values for samle plots.

\begin{tabular}{|c|c|c|c|c|c|c|c|c|c|c|c|c|c|c|c|}
\hline Serial Number & 1 & 2 & 3 & 4 & 5 & 6 & 7 & 8 & 9 & 10 & 11 & 12 & 13 & 14 & 15 \\
\hline 1. California Quail ${ }^{\text {RAI Cover Type }}$ & $\begin{array}{c}\mathbf{G} \\
1.0\end{array}$ & PEM & $\begin{array}{c}G \\
0.7\end{array}$ & $\begin{array}{c}\text { Ag-pr } \\
1.0\end{array}$ & $\begin{array}{c}\text { Ag-pf } \\
1.0\end{array}$ & Pow & $\begin{array}{c}\text { Ag-p } f \\
1.0\end{array}$ & PEM & PEM & $\mathbf{F}$ & $\mathbf{R}$ & PEM & PEM & $\begin{array}{l}\mathbf{G} \\
1.0\end{array}$ & $\begin{array}{c}\text { Ag-pf } \\
1.0\end{array}$ \\
\hline 2. Canada Goose & 0.9 & & 0.9 & 0.7 & 0.7 & 0.7 & 1.0 & & & 0.7 & & & & 0.9 & 0.7 \\
\hline $\begin{array}{l}\text { 3. Mallard } \\
\text { 4. Spotted Sandpiper }\end{array}$ & 0.0 & 0.0 & 0.1 & 0.1 & 0.0 & 0.0 & 0.2 & 0.5 & 0.0 & & & 0.9 & 0.5 & 0.3 & 0.2 \\
\hline 5. Mink & & 0.2 & & & & & & 0.2 & 0.7 & 0.4 & 0.2 & 0.9 & 0.6 & & \\
\hline $\begin{array}{l}\text { 6. Meadowlark } \\
\text { 7. Black-capped Chickadee } \\
\text { 8. Yellow Warbler }\end{array}$ & 0.0 & & 0.0 & 0.4 & 0.4 & & 0.5 & & & 0.6 & & & & 0.5 & 0.3 \\
\hline $\begin{array}{l}\text { 9. Great Blue Heron } \\
\text { 10. Downy Woodpecker }\end{array}$ & $\begin{array}{l}0.0 \\
0.0\end{array}$ & & 0.0 & 0.0 & 0.1 & 0.7 & 0.2 & & & $\begin{array}{l}0.7 \\
0.8\end{array}$ & 0.7 & & & 0.3 & 0.1 \\
\hline
\end{tabular}

\begin{tabular}{|c|c|c|c|c|c|c|c|c|c|c|c|c|c|c|c|}
\hline Serial Number & 16 & 17 & 18 & 19 & 20 & 21 & 22 & 23 & 24 & 25 & 26 & 27 & 28 & 29 & 30 \\
\hline RAI Cover Type & $\mathbf{R}$ & Ag-pr & PEM & $\mathbf{S}$ & Ag-pf & SSG & $\mathbf{F}$ & $\mathbf{F}$ & $\mathbf{F}$ & $\mathbf{F}$ & $\mathbf{H}$ & SGCM & Ag-p & PAB4 & $\mathbf{s}$ \\
\hline 1. California Quajl & & 0.9 & & 1.0 & 1.0 & 1.0 & & & & & 1.0 & & 1.0 & & 1.0 \\
\hline 2. Canada Goose & " & 0.7 & & & 0.7 & 1.0 & 1.0 & 0.7 & 0.7 & 0.7 & 0.7 & 0.7 & 0.7 & & \\
\hline 3. Mallard & & 0.5 & 0.9 & $\cdots$ & 1.0 & 0.1 & & & & & 0.0 & 0.0 & 0.4 & 0.9 & \\
\hline 4. Spotted Sandpiper & & & & & & & & & & & & 0.7 & & & \\
\hline 5. Mink & 0.2 & & 0.7 & 0.9 & & & 0.7 & 0.9 & 1.0 & 1.0 & & 0.0 & & 0.9 & 0.8 \\
\hline 6. Meadowlark & & 0.2 & 0.0 & & 0.3 & 0.0 & & & & & & & 0.5 & & \\
\hline 7. Black-capped Chickadee & & & & & & & 1.0 & 0.0 & 1.0 & 1.0 & & & & & \\
\hline 8. Yellow Warbler & & & & 0.8 & & & & & & & & & & & 0.5 \\
\hline 9. Great Blue Heron & 0.7 & 0.3 & & & 0.3 & 0.1 & 0.0 & 0.5 & 0.0 & 0.0 & & 1.0 & 0.5 & & \\
\hline 10. Downy Woodpecker & & & & & & & 0.8 & 0.0 & 0.8 & 0.8 & & & & & \\
\hline
\end{tabular}


Table C-10. Yakama Method with HSI Scores replacing step values for samle plots.

\begin{tabular}{|c|c|c|c|c|c|c|c|c|c|c|c|c|c|c|c|}
\hline Serial Number & 31 & 32 & 33 & 34 & 35 & 36 & 37 & 38 & 39 & 40 & 41 & 42 & 43 & 44 & 45 \\
\hline 1. California Quail ${ }^{\text {RAI Cover Type }}$ & $\begin{array}{l}\text { Ssc } \\
1.0\end{array}$ & $\begin{array}{c}\text { Ag-pf } \\
1.0\end{array}$ & $\begin{array}{l}\text { SSG } \\
1.0\end{array}$ & $\mathbf{F}$ & SGCM & R & PEM & $\begin{array}{l}\text { SSG } \\
1.0\end{array}$ & $\begin{array}{c}\mathbf{G} \\
1.0\end{array}$ & Pab4 & $\begin{array}{c}\text { Ag-p } \\
1.0\end{array}$ & SGCM & $\mathbf{R}$ & $\begin{array}{l}\text { Ag-p } \\
1.0\end{array}$ & $\begin{array}{l}\text { Ag-p } \\
1.0\end{array}$ \\
\hline 2. Canada Goose & 0.7 & 0.7 & 0.9 & 0.5 & 0.7 & & & 0.7 & 0.7 & & 0.7 & 0.7 & & 0.7 & 0.7 \\
\hline $\begin{array}{l}\text { 3. Mallard } \\
\text { 4. Spotted Sandpiper }\end{array}$ & 0.2 & 0.4 & 0.4 & & $\begin{array}{l}0.0 \\
0.7\end{array}$ & & 0.9 & 0.4 & 0.2 & 0.5 & 0.3 & $\begin{array}{l}0.0 \\
0.7\end{array}$ & & 0.1 & 0.1 \\
\hline 5. Mink & & & & 0.8 & 0.0 & 0.3 & 0.7 & & & 0.2 & & 0.0 & 0.6 & & \\
\hline $\begin{array}{l}\text { 6. Meadowlark } \\
\text { 7. Black-capped Chickadee } \\
\text { 8. Yellow Warbler }\end{array}$ & 0.1 & 0.5 & 0.0 & 0.9 & & & & 0.0 & 0.3 & & 0.9 & & & 0.2 & 0.2 \\
\hline $\begin{array}{l}\text { 9. Great Blue Heron } \\
\text { 10. Downy Woodpecker }\end{array}$ & 0.1 & 0.3 & 0.3 & $\begin{array}{l}0.3 \\
1.0\end{array}$ & 0.1 & 1.0 & & 0.3 & 0.2 & & 0.3 & 0.1 & 1.0 & 0.3 & 0.3 \\
\hline
\end{tabular}

\begin{tabular}{|c|c|c|c|c|c|c|c|c|c|c|c|c|c|c|c|}
\hline $\begin{array}{l}\text { Serlal Number } \\
\text { RAI Cover Type }\end{array}$ & 46 & 47 & 48 & $\begin{array}{l}49 \\
\text { Ap-p }\end{array}$ & $\begin{array}{ll}50 \\
\text { sccm }\end{array}$ & 51 & 52 & 53 & 54 & $\begin{array}{c}55 \\
\text { Appc }\end{array}$ & $\begin{array}{l}56 \\
\mathrm{H}\end{array}$ & $\begin{array}{l}57 \\
S\end{array}$ & $\begin{array}{c}58 \\
\text { Aq-pr }\end{array}$ & $\begin{array}{c}59 \\
\text { pow }\end{array}$ & $\begin{array}{l}60 \\
S\end{array}$ \\
\hline 1. California Quail & 1.0 & & 1.0 & 1.0 & & & 1.0 & & $\begin{array}{c}1.0 \\
1.0\end{array}$ & & 1.0 & 1.0 & 1.0 & & 1.0 \\
\hline 2. Canada Goose & 0.7 & & & 0.7 & 0.7 & & 0.7 & 0.7 & 0.7 & & 0.7 & & 0.7 & 0.7 & \\
\hline 3. Mallard & 0.4 & & & 0.0 & 0.2 & 0.9 & 0.5 & 0.3 & 0.4 & & 0.0 & & 0.4 & 0.3 & \\
\hline 4. Spotted Sandpiper & & & & & 0.7 & & & & & & & & & & \\
\hline 5. Mink & & 0.3 & 0.6 & & 0.0 & 0.9 & & & & & & 0.8 & & & 0.5 \\
\hline $\begin{array}{l}\text { 6. Meadowlark } \\
\text { 7. Black-capped Chickadee }\end{array}$ & 0.3 & & & 0.5 & & & 0.1 & & 0.3 & & & & 0.3 & & \\
\hline $\begin{array}{l}\text { 8. Yellow Warbler } \\
\text { 9. Great Blue Heron } \\
\text { 10. Downy Woodpecker }\end{array}$ & 0.3 & 0.7 & 0.7 & 0.3 & 0.3 & & 0.2 & 0.2 & 0.3 & & & 0.4 & 0.3 & 0.1 & 0.7 \\
\hline
\end{tabular}


Table C-10. Yakama Method with HSI Scores replacing step values for samle plots.

\begin{tabular}{|c|c|c|c|c|c|c|c|c|c|c|c|c|c|c|}
\hline $\begin{array}{r}\text { Serial Number } \\
\text { RAI Cover Type }\end{array}$ & $\begin{array}{l}\text { 6I } \\
\text { pow }\end{array}$ & $\begin{array}{l}62 \\
11\end{array}$ & $\begin{array}{c}63 \\
\text { PAB4 }\end{array}$ & $\begin{array}{c}64 \\
\text { PAB4 }\end{array}$ & $\begin{array}{l}65 \\
G\end{array}$ & $\begin{array}{l}66 \\
2\end{array}$ & $\begin{array}{l}67 \\
G\end{array}$ & $\begin{array}{c}68 \\
S\end{array}$ & $\begin{array}{c}69 \\
A \mathrm{Ag}-\mathrm{Cf}\end{array}$ & $\begin{array}{l}70 \\
\mathbf{G}\end{array}$ & $\begin{array}{l}71 \\
F\end{array}$ & $\begin{array}{c}72 \\
\text { PEM }\end{array}$ & $\begin{array}{l}73 \\
F\end{array}$ & $\begin{array}{l}74 \\
\text { SSG }\end{array}$ \\
\hline 1. Californla Quail & & 1.0 & & & 1.0 & & 1.0 & 1.0 & 1.0 & 1.0 & & & & 1.0 \\
\hline 2. Canada Goose & 0.7 & 0.7 & & & 0.7 & 0.7 & 0.7 & & & 0.7 & 0.7 & & 0.7 & 0.7 \\
\hline $\begin{array}{l}\text { 3. Mallard } \\
\text { 4. Spotted Sandpiper }\end{array}$ & 0.3 & 0.0 & 0.9 & 0.9 & 0.5 & 0.3 & 0.4 & & & 0.9 & & 0.9 & & 0.3 \\
\hline 5. Mink & & & 0.2 & 0.2 & & & & 1.0 & & & 1.0 & 0.7 & 1.0 & \\
\hline 6. Meadowlark & & & & & 0.3 & & 0.6 & & & 0.0 & & & & 0.4 \\
\hline 7. Black-capped Chickadee & & & & & & & & & & & 0.8 & & 0.8 & \\
\hline 8. Yellow Warbler & & & & & & & & 0.5 & & & & & & \\
\hline $\begin{array}{l}\text { 9. Great Blue Heron } \\
\text { 10. Downy Woodpecker }\end{array}$ & 0.1 & & & & 0.5 & 0.4 & 0.3 & & & 0.3 & $\begin{array}{l}0.0 \\
0.8\end{array}$ & & $\begin{array}{l}0.1 \\
0.2\end{array}$ & 0.3 \\
\hline
\end{tabular}

\begin{tabular}{|c|c|c|c|c|c|c|c|c|c|c|c|c|c|}
\hline Serlal Number & 76 & 77 & 78 & 79 & 80 & 81 & 82 & 83 & 84 & 85 & 86 & 87 & 88 \\
\hline RAI Cover Type & $\mathbf{R}$ & $\begin{array}{c}\mathbf{s} \\
10\end{array}$ & SGCM & $\begin{array}{c}G \\
10\end{array}$ & $\begin{array}{c}\text { Ag-cf } \\
10\end{array}$ & Ag-pf & $\begin{array}{c}\text { Ag-pf } \\
10\end{array}$ & $\mathbf{F}$ & $\begin{array}{c}\mathbf{s} \\
10\end{array}$ & PEM & SSG & $\mathbf{R}$ & 10 \\
\hline $\begin{array}{l}\text { 1. California Quail } \\
\text { 2. Canada Goose }\end{array}$ & & 1.0 & 1.0 & $\begin{array}{l}1.0 \\
1.0\end{array}$ & 1.0 & $\begin{array}{l}1.0 \\
1.0\end{array}$ & $\begin{array}{l}1.0 \\
1.0\end{array}$ & 1.0 & 1.0 & & $\begin{array}{l}1.0 \\
1.0\end{array}$ & & $\begin{array}{l}1.0 \\
0.9\end{array}$ \\
\hline 3. Mallard & & & 0.0 & 0.9 & 0.0 & 0.7 & 0.7 & & & 0.9 & 0.4 & & 0.4 \\
\hline 4. Spotted Sandpiper & & & 0.7 & & & & & & & & & & \\
\hline 5. Mink & 0.3 & 0.5 & 0.0 & & & & & 0.8 & 0.8 & 0.8 & & 0.3 & \\
\hline 6. Meadowlark & & & & 0.3 & & 0.4 & 0.5 & & & & 0.0 & & \\
\hline $\begin{array}{l}\text { 7. Black-capped Chickadee } \\
\text { 8. Yellow Warbler }\end{array}$ & & 0.5 & & & & & & 0.9 & 0.4 & & & & \\
\hline $\begin{array}{l}\text { 9. Great Blue Heron } \\
\text { 10. Downy Woodpecker }\end{array}$ & 1.0 & & 1.0 & 0.3 & & 0.2 & 0.3 & $\begin{array}{l}0.0 \\
1.0\end{array}$ & & & 0.2 & 0.3 & \\
\hline
\end{tabular}

